\title{
Resultados motivados por uma caracterização de operadores pseudo-diferenciais conjecturada por Rieffel
}

\section{Marcela Irene Merklen Olivera}

\author{
TESE APRESENTADA \\ $\mathrm{AO}$ \\ INSTITUTO DE MATEMÁTICA E ESTATÍSTICA \\ DA \\ UNIVERSIDADE DE SÃO PAULO \\ PARA \\ OBTENÇÃO DO GRAU DE DOUTOR \\ EM \\ MATEMÁTICA
}

Orientador: Prof. Dr. Severino Toscano do Rêgo Melo Co-orientadora: Prof. Dra. Cristina Cerri

Durante a elaboração deste trabalho a autora recebeu apoio financeiro do CNPq 


\section{Resultados motivados por uma caracterização de operadores pseudo-diferenciais conjecturada por Rieffel}

Este exemplar corresponde à redação final da tese devidamente corregida e defendida por Marcela Irene Merklen Olivera e aprovada pela comissão julgadora.

São Paulo, outubro de 2002.

Comissão Julgadora:

- Prof. Dr. Severino Toscano do Rego Melo (Presidente) - IME-USP

- Prof. Dr. Ruy Exel Filho - UFSC

- Prof. Dr. Milton Lopes - IMECC-UNICAMP

- Prof. Dr. José Ruidival Santos Filho - UFSCar

- Profa. Dra. Beatriz Abadie - UNIV. REP. URUGUAI 
a Alejandra, Ana, Héctor, minha familia, com muito amor 


\section{Agradecimentos}

Não há palavras que possam expressar minha gratidão por poder ter tido a oportunidade de trabalhar com o professor Dr. Severino Toscano do Rego Melo, meu orientador.

Gostaria de agradecer à professora Cristina Cerri, minha co-orientadora, que tantas vezes me ajudou a clarear as idéias.

À professora Marina Pizzotti, por sua ajuda em Teoria da Integração.

A tantos outros professores que, de um modo ou de otro, me ajudaram a concluir este trabalho.

Aos amigos tão queridos Pablo, Hernán, Liane, Daniel, Marcelo, Irene, Zé, Carolina, Marcel, Raul, Maité, Aldo, Bárbara, que me deram seu carinho e seu apoio todos estes anos.

A Cecilia Tosar, minha amiga, minha companheira, por estar sempre por perto e me ajudar tanto, não só na digitação final da tese, mas também em várias ocasiões em minha vida.

A Fernando Abadie, um grande amigo, que me ajudou tantas vezes discutindo matemática comigo. E à sua esposa, Daniella, que sempre teve palavras doces para mim.

A meus amigos no Uruguay, Gerardo, Yolanda, Mauro, Fernando que, a pesar de morar longe, sempre estiveram próximos.

A meu pai, professor Dr. Héctor Merklen, que me ajudou discutindo problemas e a quem devo a digitação da tese.

A minha mãe, professora Dra. Ana María Olivera Yamín, quem sempre me acompanhou e me ajudou.

A minha irmã, Alejandra, que sempre foi carinhosa comigo.

A minha família, em fim, sem a qual não sou nada, não pertenço a nada, que tanto me abrigou e me ajudou nestes anos e em toda minha vida.

Finalmente, a todos aqueles que não tive oportunidade de mencionar, mas com quem tive oportunidade de compartilhar minha vida nestes anos, muito obrigada. 


\title{
Resumo
}

Trabalhamos com funções definidas em $\mathbb{R}^{n}$ que tomam valores numa $C^{*}$-álgebra $A$. Consideramos o conjunto $S^{A}\left(\mathbb{R}^{n}\right)$ das funções de Schwartz, (de decrescimento rápido), com norma dada por $\|f\|_{2}=\left\|\int f(x)^{*} f(x) d x\right\|^{\frac{1}{2}}$. Denotamos por $C B^{\infty}\left(\mathbb{R}^{2 n}, A\right)$ o conjunto das funções $C^{\infty}$ com todas as suas derivadas limitadas. Provamos que os operadores pseudo-diferenciais com símbolo em $C B^{\infty}\left(\mathbb{R}^{2 n}, A\right)$ são contínuos em $S^{A}\left(\mathbb{R}^{n}\right)$ com a norma $\|\cdot\|_{2}$, fazendo uma generalização de [10]. Rieffel prova em [1] que $C B^{\infty}\left(\mathbb{R}^{n}, A\right)$ age em $S^{A}\left(\mathbb{R}^{n}\right)$ por meio de um produto deformado, induzido por uma matriz antisimétrica, $J$, como segue: $L_{F} g(x)=F \times_{J} g(x)=\int e^{2 \pi i u v} F(x+J u) g(x+v) d u d v$, (integral oscilatória).

Dizemos que um operador $S$ é Heisenberg-suave se as aplicações $z \mapsto T_{-z} S T_{z}$ e $\zeta \mapsto M_{-\zeta} S M_{\zeta}, z, \zeta \in \mathbb{R}^{n}$, são $C^{\infty}$; onde $T_{z} g(x)=g(x-z)$ e $M_{\zeta} g(x)=e^{i \zeta x} g(x)$. No final do capítulo 4 de [1] , Rieffel propõe uma conjectura: que todos os operadores "adjuntáveis" em $S^{A}\left(\mathbb{R}^{n}\right)$, Heisenberg-suaves, que comutam com a representação regular à direita de $C B^{\infty}\left(\mathbb{R}^{n}, A\right), R_{G} f=f \times_{J} G$, são os operadores do tipo $L_{F}$. Provamos este resultado para o caso $A=\varnothing,[\mathbf{1 4}]$, usando a caracterização de Cordes $[\mathbf{1 7}]$ dos operadores Heisenberg-suaves em $L^{2}\left(\mathbb{R}^{n}\right)$ como sendo os operadores pseudo-diferenciais com símbolo em $C B^{\infty}\left(\mathbb{R}^{2 n}\right)$. Também é provado neste trabalho que, se vale uma generalização natural da caracterização de Cordes, a conjectura de Rieffel é verdadeira.

\begin{abstract}
We work with functions defined on $\mathbb{R}^{n}$ with values in a $C^{*}$-algebra $A$. We consider the set $S^{A}\left(\mathbb{R}^{n}\right)$ of Schwartz functions (rapidly decreasing), with norm given by $\|f\|_{2}=$ $\left\|\int f(x)^{*} f(x) d x\right\|^{\frac{1}{2}}$. We denote $C B^{\infty}\left(\mathbb{R}^{2 n}, A\right)$ the set of functions which are $C^{\infty}$ and have all their derivatives bounded. We prove that pseudo-differential operators with symbol in $C B^{\infty}\left(\mathbb{R}^{2 n}, A\right)$ are continuous on $S^{A}\left(\mathbb{R}^{n}\right)$ with the norm $\|\cdot\|_{2}$, thus generalizing the result in [10]. Rieffel proves in $[\mathbf{1}]$ that $C B^{\infty}\left(\mathbb{R}^{n}, A\right)$ acts on $S^{A}\left(\mathbb{R}^{n}\right)$ through a deformed product induced by an anti-symmetric matrix, J, as follows: $L_{F} g(x)=F \times_{J} g(x)=$ $\int e^{2 \pi i u v} F(x+J u) g(x+v) d u d v$ (an oscillatory integral).

We say that an operator $S$ is Heisenberg-smooth if the maps $z \mapsto T_{-z} S T_{z}$ and $\zeta \mapsto M_{-\zeta} S M_{\zeta}, z, \zeta \in \mathbb{R}^{n}$ are $C^{\infty}$; where $T_{z} g(x)=g(x-z)$ and where $M_{\zeta} g(x)=$ $e^{i \zeta x} g(x)$. At the end of chapter 4 of [1] , Rieffel proposes a conjecture: that all "adjointable" operators in $S^{A}\left(\mathbb{R}^{n}\right)$ that are Heisenberg-smooth and that commute with the right-regular representation of $C B^{\infty}\left(\mathbb{R}^{n}, A\right), R_{G} f=f \times_{J} G$, are operators of type $L_{F}$. We proved this result for the case $A=C$ in [14], using Cordes' characterization of Heisenberg-smooth operators on $L^{2}\left(\mathbb{R}^{n}\right)$ as being the pseudo-differential operators with symbol in $C B^{\infty}\left(\mathbb{R}^{2 n}\right)$. It is also proved in this thesis that, if a natural generalization of Cordes' characterization is valid, then the Rieffel conjecture is true.
\end{abstract}




\section{Sumário}

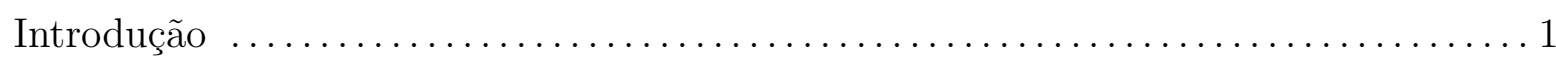

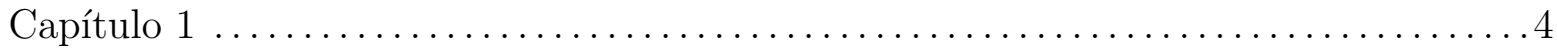

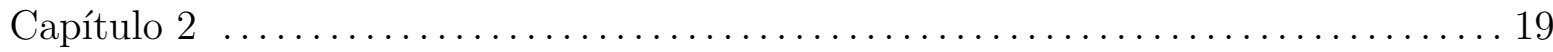

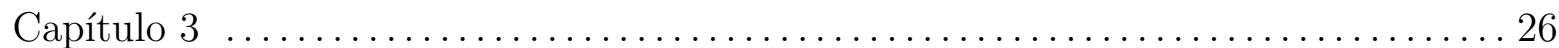

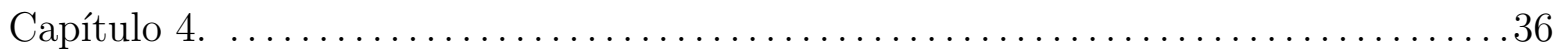

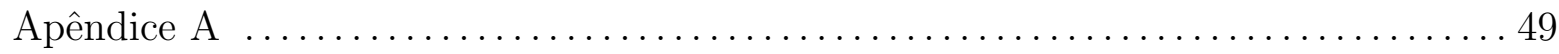

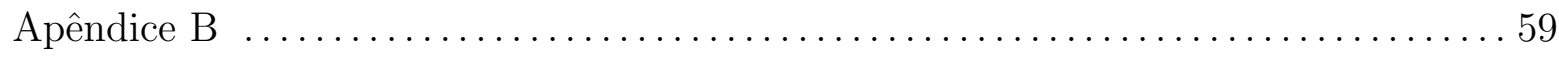

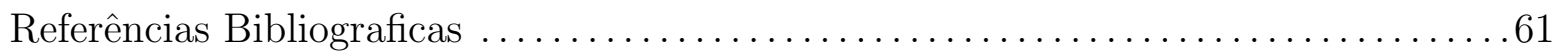




\section{Introdução}

Consideramos uma $C^{*}$-álgebra, $A$, e funções de $\mathbb{R}^{n}$ em $A$. Em particular, trabalhamos com o conjunto das funções de Schwartz, $S^{A}\left(\mathbb{R}^{n}\right)$, que consiste nas funções $f: \mathbb{R}^{n} \rightarrow A, C^{\infty}$, tais que $\sup _{x \in \mathbb{R}^{n}}\left\|x^{\alpha} \partial^{\beta} f(x)\right\|$ é limitado para quaisquer multiíndices $\alpha$ e $\beta,[\mathbf{1}$, capítulo 3]. Neste conjunto definimos o "produto interno" $\langle f, g\rangle=$ $\int f(x)^{*} g(x) d x,[\mathbf{1},(4.1)] . S^{A}\left(\mathbb{R}^{n}\right)$, com a norma induzida por este produto interno, $\|f\|_{2}=\|<f, f>\|^{\frac{1}{2}}$, é um pré-módulo de Hilbert (ver definição 1.2). Denotamos por $\overline{S^{A}\left(\mathbb{R}^{n}\right)}$ o módulo de Hilbert correspondente, isto é o completamento de $S^{A}\left(\mathbb{R}^{n}\right)$ com respeito a esta norma.

Observamos que o espaço de Fréchet $C B^{\infty}\left(\mathbb{R}^{n}, A\right)$, das funções $C^{\infty}$ limitadas, com todas as derivadas limitadas, age em $S^{A}\left(\mathbb{R}^{n}\right)$ do seguinte modo: dadas $F \in$ $C B^{\infty}\left(\mathbb{R}^{n}, A\right)$ e $g \in S^{A}\left(\mathbb{R}^{n}\right), L_{F}(g)=F \times_{J} g \in S^{A}\left(\mathbb{R}^{n}\right)$, como vemos em [1, (3.3)]. Aqui Rieffel define o produto deformado, $\times_{J}$, para uma matriz, $J, n \times n$, anti-simétrica, em $C B^{\infty}\left(\mathbb{R}^{n}, A\right)$, como segue: $F \times{ }_{J} G(x)=\iint F(x+J u) G(x+v) e(u \cdot v) d u d v$, onde $e(u \cdot v)=\exp (2 \pi i u \cdot v)$ e a integral acima é uma integral oscilatória, [1, (1.3)]. O operador $L_{F}$ definido acima possui "adjunto", [1, (4.2)], (com respeito ao "produto interno" acima definido), e é contínuo no módulo de Hilbert $\overline{S^{A}\left(\mathbb{R}^{n}\right)},[\mathbf{1},(4.7)]$.

Seja $B^{*}\left(\overline{S^{A}\left(\mathbb{R}^{n}\right)}\right)$ o conjunto dos operadores lineares contínuos em $\overline{S^{A}\left(\mathbb{R}^{n}\right)}$ que possuem adjunto. Como no caso escalar, [2, capítulo 8], consideramos em $B^{*}\left(\overline{S^{A}\left(\mathbb{R}^{n}\right)}\right)$ a ação do grupo de Heisenberg como segue: $(z, \zeta, \varphi) \mapsto E_{z, \zeta, \varphi} \Lambda E_{z, \zeta, \varphi}^{-1}, z, \zeta \in \mathbb{R}^{n}, \varphi \in \mathbb{R}$, para $\Lambda \in B^{*}\left(\overline{S^{A}\left(\mathbb{R}^{n}\right)}\right)$, onde $E_{z, \zeta, \varphi}=e^{i \varphi} M_{\zeta} T_{z}$, com $T_{z} u(x)=u(x-z), M_{\zeta} u(x)=$ $e^{\zeta x} u(x), \quad u \in S^{A}\left(\mathbb{R}^{n}\right)$. Notamos que $E_{z, \zeta \varphi}^{-1} \Lambda E_{z, \zeta, \varphi}$ independe de $\varphi$. Tomamos, então, $\varphi=0$ e usamos a notação $E_{z, \zeta}=E_{z, \zeta, 0}$ e $\Lambda_{z, \zeta}=E_{z, \zeta}^{-1} \Lambda E_{z, \zeta}$. Dizemos que o operador $\Lambda$ é Heisenberg-suave se a aplicação $(z, \zeta) \mapsto \Lambda_{z, \zeta}\left((z, \zeta) \in \mathbb{R}^{2 n}\right)$ é $C^{\infty}$.

Assim como definimos a ação à esquerda de $C B^{\infty}\left(\mathbb{R}^{n}, A\right)$ em $S^{A}\left(\mathbb{R}^{n}\right)$ por $L_{F}(g)=$ $F \times{ }_{J} g$, podemos definir a ação à direita de $C B^{\infty}\left(\mathbb{R}^{n}, A\right)$ em $S^{A}\left(\mathbb{R}^{n}\right)$ por $R_{G}(f)=$ $f \times{ }_{J} G, G \in C B^{\infty}\left(\mathbb{R}^{n}, A\right)$ e $f \in S^{A}\left(\mathbb{R}^{n}\right)$. $R_{G}$ é um operador contínuo no espaço de Banach $\overline{S^{A}\left(\mathbb{R}^{n}\right)}$, embora não seja homomorfismo de módulos à direita. 
No final do capítulo 4 de [1] , Rieffel propõe uma questão que podemos enunciar do seguinte modo: o conjunto dos operadores em $B^{*}\left(\overline{S^{A}\left(\mathbb{R}^{n}\right)}\right)$, Heisenberg-suaves, que comutam com $R_{G}$, para toda $G \in C B^{\infty}\left(\mathbb{R}^{n}, A\right)$, é o conjunto dos operadores $L_{F}, F \in$ $C B^{\infty}\left(\mathbb{R}^{n}, A\right)$.

No primeiro capítulo deste trabalho demonstramos esta conjectura para o caso em que $A$ é a $C^{*}$-álgebra dos números complexos, [14]. Para isto, usamos a caracterização feita por Cordes em $[\mathbf{1 7}]$ dos operadores lineares contínuos em $L^{2}\left(\mathbb{R}^{n}\right)$ (no caso em que $\left.A=\not C, \overline{S^{A}\left(\mathbb{R}^{n}\right)}=L^{2}\left(\mathbb{R}^{n}\right)\right)$ que são Heisenberg-suaves. Cordes prova que estes são os operadores $a(x, D)$, dados por um símbolo, uma função $a \in C B^{\infty}\left(\mathbb{R}^{2 n}\right)$, do seguinte modo: $a(x, D) u(x)=\iint(2 \pi)^{-n} e^{i(x-y) \xi} a(x, \xi) u(y) d y d \xi$, onde $u \in C_{0}^{\infty}\left(\mathbb{R}^{n}\right)$. Provamos, então, que, se $a(x, D)$ comuta com $R_{G}$ para toda $G$ em $C B^{\infty}\left(\mathbb{R}^{n}\right)$, então se $F(x)=a(x, 0), a(x, D)=L_{F}$.

No capítulo 2, precisamos supor que $A$ é separável para provar um resultado interessante. Na verdade, se $A$ tiver unidade, a separabilidade não é necessária, pois só a necessitamos para ter uma aproximação da unidade em $A$ dada por uma seqüência. Dizemos que um operador $\Lambda$ em $B^{*}\left(\overline{S^{A}\left(\mathbb{R}^{n}\right)}\right)$ é suave por translações se a aplicação $z \mapsto \Lambda_{z}, z \in \mathbb{R}^{n}$ é $C^{\infty}$, onde temos que $\Lambda_{z}=T_{z}^{-1} \Lambda T_{z}$, usando a notação anterior. Neste capítulo, provamos que se $\Lambda$ é suave por translações e comuta com $R_{g}$ para qualquer $g$ em $S^{A}\left(\mathbb{R}^{n}\right)$, então $\Lambda$ é Heisenberg-suave.

O capítulo 3 consiste em uma generalização do teorema de Calderón-Vaillancourt, [10], baseada no teorema 3.14 de [11]. Provamos que um operador no módulo $\overline{S^{A}\left(\mathbb{R}^{n}\right)}$ dado por um símbolo (como no caso escalar, [2]) é contínuo. Aqui estamos nos referindo aos operadores $a(x, D), a \in C B^{\infty}\left(\mathbb{R}^{2 n}, A\right)$ dados por $a(x, D) u(x)=\iint(2 \pi)^{-n} e^{i(x-y) \xi} a(x, \xi)$ $u(y) d y d \xi$, para $u \in S^{A}\left(\mathbb{R}^{n}\right)$. A integral acima é uma integral oscilatória. Novamente supomos que $A$ é separável, para poder aplicar o Teorema de Convergência Dominada, e outras propriedades, para a integral de Bochner, [13, (E.6)], e também para usar que a transformada de Fourier é um operador "unitário" em $S^{A}\left(\mathbb{R}^{n}\right)$ (ver apêndice B) e que $S^{A}\left(\mathbb{R}^{n}\right)$ é denso em $L^{2}\left(\mathbb{R}^{n}, A\right)$ (ver apêndice $\mathrm{A}$ ).

Usando os resultados dos capítulos 2 e 3, no capítulo 4, provamos que uma gene ralização natural, para uma $C^{*}$-álgebra separável qualquer, $A$, da caracterização de Cordes implicaria que a conjectura de Rieffel é verdadeira. Isto generaliza os resultados 
de [14]; lá nós provamos que a caracterização de Cordes implica a conjectura de Rieffel, no caso $A=\varnothing$, através de um argumento que usa o núcleo de Schwartz de um operador pseudo-diferencial. No capítulo 4, os argumentos que envolvem distribuições temperadas no capítulo 1, serão substituídos pela proposição 4.11, que se trata, na verdade, de uma extensão imediata, para o caso $A$ qualquer, de um trecho da demonstração na caracterização de Cordes. 


\section{Capítulo 1}

Neste capítulo vamos apresentar a conjectura proposta por Rieffel no final de [1, Capítulo 4] para uma $C^{*}$-álgebra qualquer e veremos uma resposta para o caso da $C^{*}$-álgebra dos números complexos.

Seja $A$ uma $C^{*}$-álgebra. Consideremos o espaço das funções contínuas e limitadas de $\mathbb{R}^{n}$ em $A$, que possuem derivadas de todas as ordens que são contínuas e limitadas.

$$
C B^{\infty}\left(\mathbb{R}^{n}, A\right)=\left\{F: \mathbb{R}^{n} \longrightarrow A ;\left\|\partial^{\alpha} F\right\|_{\infty} \leq \infty, \forall \alpha\right\}
$$

onde $\alpha$ é um multi-índice, $\alpha=\left(\alpha_{1}, \alpha_{2}, \cdots, \alpha_{n}\right) \in \mathbb{N}^{n}$ e

$$
\begin{gathered}
\partial^{\alpha} F=\partial^{\alpha_{1}} \partial^{\alpha_{2}} \cdots \partial^{\alpha_{n}} F, \\
\left\|\partial^{\alpha} F\right\|_{\infty}=\sup _{x \in \mathbb{R}^{n}}\left\|\partial^{\alpha} F(x)\right\|,
\end{gathered}
$$

onde esta última é a norma em $A$.

Consideremos em $C B^{\infty}\left(\mathbb{R}^{n}, A\right)$ as seminormas

$$
\|F\|_{j}=\sup _{|\alpha| \leq j}\left\|\partial^{\alpha} F\right\|_{\infty}
$$

onde $|\alpha|=\alpha_{1}+\alpha_{2}+\cdots+\alpha_{n}$.

Deste modo vemos que $C B^{\infty}\left(\mathbb{R}^{n}, A\right)$ é um espaço de Fréchet. 
Dada $J$, uma matriz anti-simétrica $n \times n$, definimos o produto deformado $\times_{J} \mathrm{em}$ $C B^{\infty}\left(\mathbb{R}^{n}, A\right)$ como segue:

$$
F \times{ }_{J} G(x)=\int F(x+J u) G(x+v) e^{i u \cdot v} d u d v,
$$

onde $\not u=(2 \pi)^{-\frac{n}{2}} d u, \not d v=(2 \pi)^{-\frac{n}{2}} d v$. Notemos que a rigor, considerando a definição de Rieffel do capítulo 3 de $[\mathbf{1}]$, nosso $\times_{J}$ é seu $\times_{2 \pi J}$.

A integral acima é uma integral oscilatória. Isto é, se temos $\psi_{m} \in C_{c}^{\infty}\left(\mathbb{R}^{n}\right)$, que satisfaz

(i) $\sup _{x \in \mathbb{R}^{n}}\left|\partial^{\alpha} \psi_{m}(x)\right|<c_{\alpha}$, para cada multi-índice $\alpha$, para todo $m \in \mathbb{N}$, onde $c_{\alpha} \in \mathbb{R}^{+}$é uma constante que depende só de $\alpha$.

(ii) $\quad \psi_{m}(x)=1$, se $x$ pertence à bola de centro na origem e raio $r_{m}, B\left(O, r_{m}\right)$, onde $r_{m}$ cresce para infinito;

e também $\psi_{k}^{\prime} \in C_{c}^{\infty}\left(\mathbb{R}^{n}\right)$, que satisfaz $(i)$ e $(i i)$, então $\int F(x+J u) G(x+v) e^{i u \cdot v} d u d v=\lim _{k, m \rightarrow \infty} \iint F(x+J u) G(x+v) \psi_{m}(u) \psi_{k}^{\prime}(v) e^{i u \cdot v} d u d v$. Prova-se que o limite existe e é independente da escolha de $\psi_{m}$ e $\psi_{k}^{\prime}([\mathbf{1},(2.4)])$.

O produto deformado está bem definido em $C B^{\infty}\left(\mathbb{R}^{n}, A\right)([\mathbf{1}, 2.2])$.

Proposição 1.1. Dadas $F, G \in C B^{\infty}\left(\mathbb{R}^{n}, A\right)$, temos que

$$
F \times{ }_{J} G(x)=\int e^{i u(x-v)} F(x-J u) G(v) d v d u .
$$

Demonstração. Pelo visto acima, temos que

$$
\begin{aligned}
F & \times{ }_{J} G(x)=\lim _{m, k} \iint e^{i u w} F(x+J u) G(x+w) \psi_{m}(u) \psi_{k}^{\prime}(w) d u d w= \\
& =\lim _{m, k \rightarrow \infty} \iint e^{i u(v-x)} F(x+J u) G(v) \psi_{m}(u) \psi_{k}^{\prime}(v-x) d u d v= \\
& =\lim _{m, k \rightarrow \infty} \iint e^{-i u(v-x)} F(x-J u) G(v) \psi_{m}(-u) \psi_{k}^{\prime}(v-x) d v d u
\end{aligned}
$$

pela proposição A.18 e pelo Teorema de Fubini. Para $x$ fixo, $\psi_{m}(-u)$ e $\psi_{k}^{\prime}(v-x)$ satisfazem as condições (i) e (ii) acima, então temos:

$$
F \times{ }_{J} G(x)=\int e^{i u(x-v)} F(x-J u) G(v) d u d v .
$$


Interessa-nos $C B^{\infty}\left(\mathbb{R}^{n}, A\right)$ agindo num espaço com produto interno. Assim, vamos considerar as funções de Schwartz. Seja $S^{A}\left(\mathbb{R}^{n}\right)$ o subespaço de $C B^{\infty}\left(\mathbb{R}^{n}, A\right)$ das funções tais que o produto de suas derivadas por polinômios em $\mathbb{R}^{n}$ são limitadas.

$$
S^{A}\left(\mathbb{R}^{n}\right)=\left\{f: \mathbb{R}^{n} \rightarrow A /\left\|x^{\alpha} \partial^{\beta} f\right\|_{\infty}<\infty\right\}
$$

para $\alpha$ e $\beta$ multi-índices.

Dadas $F \in C B^{\infty}\left(\mathbb{R}^{n}, A\right)$ e $g \in S^{A}\left(\mathbb{R}^{n}\right)$, prova-se que $F \times_{J} g$ e $g \times_{J} F$ estão em $S^{A}\left(\mathbb{R}^{n}\right)([\mathbf{1}, 3.3])$.

Definição 1.2. Um pré-módulo de Hilbert sobre $A$ é um espaço vetorial sobre $C, E$, que é também um módulo à direita equipado com uma aplicação $\langle\cdot, \cdot>$ : $E \times E \rightarrow A$ que é linear na segunda variável e satisfaz, para $a \in A, x, y \in E$, as seguintes propriedades:

(i) $<x, y a>=<x, y>a$

(ii) $\left\langle x, y>^{*}=<y, x>\right.$

(iii) $<x, x>\geq 0$

(iv) se $\langle x, x>=0$, então $x=0$.

Em $E$ definimos $\|x\|=\|<x, x>\|^{\frac{1}{2}}, \quad x \in E$.

Prova-se que assim $E$ é um espaço normado ([7, lema 1.1.2]). Se $E$ é completo com respeito a esta norma, dizemos que $E$ é um módulo de Hilbert.

Nota 1.3. Podemos ler mais sobre módulos de Hilbert em [7, capítulo 1].

Consideremos em $S^{A}\left(\mathbb{R}^{n}\right)$ o produto interno

$$
<f, g>=\int f(x)^{*} g(x) d x .
$$

É fácil ver que $S^{A}\left(\mathbb{R}^{n}\right)$ com este produto interno é um pré-módulo de Hilbert sobre $A$. A norma correspondente é

$$
\|f\|_{2}=\|<f, f>\|^{\frac{1}{2}}, \quad f \in S^{A}\left(\mathbb{R}^{n}\right) .
$$

Denotemos por $L$ a ação à esquerda de $C B^{\infty}\left(\mathbb{R}^{n}, A\right)$ em $S^{A}\left(\mathbb{R}^{n}\right)$ dada pelo produto deformado como segue: 


$$
L_{F} g=F \times_{J} g \quad F \in C B^{\infty}\left(\mathbb{R}^{n}, A\right), g \in S^{A}\left(\mathbb{R}^{n}\right) .
$$

Vemos em $[\mathbf{1},(4.7)]$ que $L_{F}$ é um operador contínuo em $S^{A}\left(\mathbb{R}^{n}\right)$ como pré-módulo de Hilbert. No capítulo 3 deste trabalho, veremos uma demonstração do Teorema de Calderón-Vaillancourt [10] para operadores pseudo-diferenciais com símbolo tomando valores em $A$, que também implica que $L_{F}$ é contínuo.

Além disso, o operador $L_{F}$ é "adjuntável", isto é, existe $\left(L_{F}\right)^{*}$, operador limitado em $S^{A}\left(\mathbb{R}^{n}\right)$, tal que $\left\langle L_{F} u, v>=<u,\left(L_{F}\right)^{*} v>\right.$, para todo $u, v \in S^{A}\left(\mathbb{R}^{n}\right)$. De fato, denotando por $F^{*}$ a função $F^{*}(x)=F(x)^{*}$, demonstra-se $\left(\operatorname{ver}[\mathbf{1},(4.2)]\right.$ ) que $\left(L_{F}\right) *=$ $L_{F^{*}}$.

Do mesmo modo, denotemos por $R$ a ação à direita de $C B^{\infty}\left(\mathbb{R}^{n}, A\right)$ em $S^{A}\left(\mathbb{R}^{n}\right)$ :

$$
R_{G} f=f \times{ }_{J} G \quad f \in S^{A}\left(\mathbb{R}^{n}\right), G \in C B^{\infty}\left(\mathbb{R}^{n}, A\right) .
$$

Observemos que $R_{G}$ não é um homomorfismo no módulo de Hilbert $S^{A}\left(\mathbb{R}^{n}\right)$, pois, dado $a \in A$,

$$
\begin{aligned}
& R_{G}(f a)(x)=\int f(x+J u) a G(x+v) e^{i u \cdot v} d u d v \\
& R_{G}(f) a(x)=\int f(x+J u) G(u+v) a e^{i u \cdot v} d u d v .
\end{aligned}
$$

Ou seja, se $A$ não é comutativa, não vale necessáriamente que $R_{G}(f) a=R_{G}(f a)$.

Como feito em [1, (4.4)], temos que, dada $g \in S^{A}\left(\mathbb{R}^{n}\right)$, o operador $R_{g}$ é contínuo no espaço de Banach $\overline{S^{A}\left(\mathbb{R}^{n}\right)}$, o completamento de $S^{A}\left(\mathbb{R}^{n}\right)$ com a norma $\|\cdot\|_{2}$.

Denotemos com $\mathcal{B}^{*}\left(\overline{S^{A}\left(\mathbb{R}^{n}\right)}\right)$ os operadores em $\overline{S^{A}\left(\mathbb{R}^{n}\right)}$ que possuem adjunto. Como feito por Cordes, para o caso $A=C$ ([2, Cap.8,Sec.2]), consideremos a ação do grupo de Heisenberg em $\mathcal{B}^{*}\left(\overline{S^{A}\left(\mathbb{R}^{n}\right)}\right)$. O Grupo de Heisenberg é o espaço $\mathbb{R}^{2 n+1}$ equipado com a operação

$$
(z, \zeta, \varphi) \circ\left(z^{\prime}, \zeta^{\prime}, \varphi^{\prime}\right)=\left(z+z^{\prime}, \zeta+\zeta^{\prime}, \varphi+\varphi^{\prime}-\zeta^{\prime} z\right)
$$

unidade $=(0,0,0)$. 
Para $z, \zeta \in \mathbb{R}^{n}$, sejam os operadores $T_{z}, M_{\zeta} \in \mathcal{B}^{*}\left(\overline{S^{A}\left(\mathbb{R}^{n}\right)}\right)$ dados por

$$
\begin{aligned}
& T_{z} f(x)=f(x-z) \\
& M_{\zeta} f(x)=e^{i \zeta x} f(x)
\end{aligned}
$$

para $f \in S^{A}\left(\mathbb{R}^{n}\right)$. Notemos que $T_{z}$ e $M_{\zeta}$ são "unitários" com respeito ao "produto interno" $<\cdot, \cdot>$.

Observemos que vale

$$
T_{-z} M_{\zeta} T_{z} M_{-\zeta}=e^{i z \zeta} \quad M_{\zeta}^{*}=M_{-\zeta} \quad T_{z}^{*}=T_{-z}
$$

Assim, o sub-grupo dos operadores unitários em $\mathcal{B}^{*}\left(\overline{S^{A}\left(\mathbb{R}^{n}\right)}\right)$ gerado pelos operadores $T_{z}$ e $M_{\zeta}, G H$, consiste no conjunto dos operadores

$$
E_{z, \zeta, \varphi}=e^{i \varphi} M_{\zeta} T_{z}, \quad \varphi \in \mathbb{R}, z, \zeta \in \mathbb{R}^{n}
$$

Deste modo, temos uma aplicação do grupo de Heisenberg em $G H$, bem definida (não injetora), que a cada $(z, \zeta, \varphi) \in \mathbb{R}^{2 n+1}$ associa o operador $E_{z, \zeta, \varphi}$. A partir de agora, estaremos pensando no grupo $G H$ quando mencionarmos o grupo de Heisenberg.

Consideremos então a ação do grupo de Heisenberg em $\mathcal{B}^{*}\left(\overline{S^{A}\left(\mathbb{R}^{n}\right)}\right)$ por conjugação. Para $T \in \mathcal{B}^{*}\left(\overline{S^{A}\left(\mathbb{R}^{n}\right)}\right)$ seja $T_{z, \zeta}=E_{z, \zeta, \varphi}^{-1} T E_{z, \zeta, \varphi}$.

Observemos que $T_{z, \zeta}$ independe de $\varphi$. De agora em diante tomemos $\varphi=0 \mathrm{e}$ denotemos por $E_{z, \zeta}$ o operador $E_{z, \zeta, 0}$. No final de [1, Cap.4], Rieffel faz uma conjectura que, no presente contexto, pode ser enunciada como segue:

$O$ conjunto dos operadores $L_{F}$ com $F$ em $C B^{\infty}\left(\mathbb{R}^{n}, A\right)$ é o conjunto dos operadores adjuntáveis em $\overline{S^{A}\left(\mathbb{R}^{n}\right)}$ que são suaves pela ação do grupo de Heisenberg e comutam com $R_{G}$ para toda $G$ em $C B^{\infty}\left(\mathbb{R}^{n}, A\right)$, ou seja os operadores $T \in \mathcal{B}^{*}\left(\overline{S^{A}\left(\mathbb{R}^{n}\right)}\right)$ tais que a aplicação $(z, \zeta) \rightarrow T_{z, \zeta}$ é $C^{\infty}$ para $(z, \zeta) \in \mathbb{R}^{2 n}$ e $\left[T, R_{G}\right]=0, \forall G \in C B^{\infty}\left(\mathbb{R}^{n}, A\right)$.

Definição 1.4. Dado $T \in \mathcal{B}^{*}\left(\overline{S^{A}\left(\mathbb{R}^{n}\right)}\right)$, dizemos que $T$ é Heisenberg-suave se a aplicação $(z, \zeta) \rightarrow T_{z, \zeta}$ é $C^{\infty}$, para $(z, \zeta) \in \mathbb{R}^{2 n}$. 
Proposição 1.5. Dada $F \in C B^{\infty}\left(\mathbb{R}^{n}, A\right)$, o operador $L_{F}$ é Heisenberg-suave.

Demonstração. Dada $g \in S^{A}\left(\mathbb{R}^{n}\right)$, temos, pela proposição 1.1

$$
L_{F} g(x)=\int e^{i u(x-v)} F(x-J u) g(v) d v d u .
$$

Para $z, \zeta \in \mathbb{R}^{n}$, por argumentos similares aos usados na proposição 1.1,

$$
\begin{gathered}
E_{z, \zeta}^{-1} L_{F} E_{z, \zeta} g(x)=T_{-z} M_{-\zeta} L_{F} E_{z, \zeta} g(x)=e^{-i \zeta(x+z)} L_{F} E_{z, \zeta} g(x+z)= \\
=e^{-i \zeta(x+z)} L_{F} E_{x, \zeta} g(x+z)=e^{-i \zeta(x+z)} \int e^{i u(x+z-v)} F(x+z-J u) E_{z, \zeta} g(v) d v d u= \\
=e^{-i \zeta(x+z)} \int e^{i u(x+z-v)} F(x+z-J u) e^{i \zeta v} g(v-z) d v d u= \\
=e^{-i \zeta(x+z)} \int e^{i u(x-w)} F(x+z-J u) e^{i \zeta(w+z)} g(w) d w d u= \\
=e^{-i \zeta x} \int e^{i u x} e^{-i w(u-\zeta)} F(x+z-J u) g(w) d w d u= \\
=e^{-i \zeta x} \int e^{i(\xi+\zeta) x} e^{-i w \xi} F(x+z-J(\xi+\zeta)) g(w) d w d \xi= \\
=\int e^{i \xi(x-w)} F(x+z-J(\xi+\zeta)) g(w) d w d \xi .
\end{gathered}
$$

(Notemos que esta manipulação formal é válida também para integrais oscilatórias.)

Consideremos o quociente:

$$
\frac{\left(L_{F}\right)_{x+t e_{j}, \zeta}-\left(L_{F}\right)_{z, \zeta}}{t}=\frac{E_{z, \zeta}^{-1}\left(L_{F}\right)_{t e_{j}, 0} E_{z, \zeta}-E_{z, \zeta}^{-1} L_{F} E_{z, \zeta}}{t}=E_{z, \zeta}^{-1}\left[\frac{\left(L_{F}\right)_{t e_{j}, 0}-L_{F}}{t}\right] E_{z, \zeta}
$$

onde $e_{j}=(0, \cdots, 1,0, \cdots, 0) \in \mathbb{R}^{n}$.

E, pelo visto acima,

$$
\begin{gathered}
{\left[\frac{\left(L_{F}\right)_{t e_{j}, 0}-L_{F}}{t}\right](g)(x)=} \\
=\int e^{i \xi(x-w)}\left[\frac{F\left(x+t e_{j}-J \xi\right)-F(x-J \xi)}{t}\right] g(w) d w d \xi .
\end{gathered}
$$

Por $[\mathbf{1},(4.7)]$ temos que existem $k \in \mathbb{N}$ e uma constante $c_{k} \in \mathbb{R}^{+}$, tais que $\left\|L_{G}\right\| \leq c_{k}\|G\|_{2 k}$, para toda $G \in C B^{\infty}\left(\mathbb{R}^{n}, A\right)$. Sejam $H_{t}(y)=\frac{F\left(y+t e_{j}\right)-F(y)}{t}$ e $\partial_{e_{j}} F(y)=$ 
$\lim _{t \rightarrow 0} H_{t}(y)$. Como $F \in C B^{\infty}\left(\mathbb{R}^{n}, A\right)$, temos que para todo $l \in \mathbb{N}, \lim _{t \rightarrow 0} \| H_{t}-$ $\partial_{e_{j}} F \|_{l}=0$. De fato, dado $l \in \mathbb{N},\left\|H_{t}-\partial_{e_{j}} F\right\|_{l}=\sup _{|\alpha| \leq l}\left\|\partial^{\alpha}\left(H_{t}-\partial_{e_{j}} F\right)\right\|_{\infty}$. Pelo Teorema Fundamental do Cálculo, A.14,

$$
\begin{gathered}
H_{t}(y)-\partial_{e_{j}} F(y)=\frac{F\left(y+t e_{j}\right)-F(y)}{t}-\partial_{e_{j}} F(y)= \\
=\int_{[0, t]}\left[\frac{\partial_{e_{j}} F\left(y+h e_{j}\right)}{t}-\partial_{e_{j}} F(y)\right] d h=\int_{[0, t]} \frac{1}{t}\left[\partial_{e_{j}} F\left(y+h e_{j}\right)-\partial_{e_{j}} F(y)\right] d h= \\
=\int_{[0, t]} \frac{1}{t} \int_{[0, h]} \partial_{e_{j}}^{2} F\left(y+s e_{j}\right) d s d h .
\end{gathered}
$$

Logo, $\left\|H_{t}(y)-\partial_{e_{j}} F(y)\right\| \leq t\left\|\partial_{e_{j}}^{2} F\right\|_{\infty}, \quad \forall y \in \mathbb{R}^{n}$.

De modo análogo, temos que

$$
\left\|\partial^{\alpha}\left(H_{t}-\partial_{e_{j}} F\right)\right\|_{\infty} \leq t\left\|\partial_{e_{j}}^{2} \partial^{\alpha} F\right\|_{\infty}, \quad|\alpha| \leq l,
$$

e, assim, $\lim _{t \rightarrow 0}\left\|H_{t}-\partial_{e_{j}} F\right\|_{l}=0, \quad \forall l \in \mathbb{N}$.

Deste modo, $\left\|L_{H_{t}}-L_{\partial_{e_{j}} F}\right\| \leq c_{k}\left\|H_{t}-\partial_{e_{j}} F\right\|_{2 k}$ e, portanto, $\lim _{t \rightarrow 0}\left\|L_{H_{t}}-L_{\partial_{e_{j}} F}\right\|=$ 0 , o que implica que existe a derivada parcial $\partial_{z_{j}}\left(L_{F}\right)_{z, \zeta}=L_{\partial_{e_{j}} F}$. Como obtivemos um operador do mesmo tipo, o processo pode ser repetido indefinidamente. Logo, $L_{F}$ é Heisenberg-suave.

Proposição 1.6. Dadas $F, G \in C B^{\infty}\left(\mathbb{R}^{n}, A\right)$, quaisquer, $\left[L_{F}, R_{G}\right]=0$.

Demonstração. Consideremos $u \in S^{A}\left(\mathbb{R}^{n}\right)$.

$$
\begin{aligned}
& L_{F} R_{G} u=F \times_{J} R_{G} u=F \times_{J}\left(u \times_{J} G\right) \\
& R_{G} L_{F} u=L_{F} u \times{ }_{J} G=\left(F \times{ }_{J} u\right) \times{ }_{J} G .
\end{aligned}
$$

Como o producto deformado é associativo $([\mathbf{1}, 2.14])$, temos $\left[L_{F}, R_{G}\right]=0$.

1.7. Deste modo, dado $F \in C B^{\infty}\left(\mathbb{R}^{n}, A\right)$, temos que $L_{F}$ é um operador Heisenbergsuave que comuta com $R_{G}$ para todo $G \in C B^{\infty}\left(\mathbb{R}^{n}, A\right)$.

Observação 1.8. Pelo visto na proposição 1.1, $L_{F} g(x)=\int e^{i u(x-v)} F(x-J u) g(v) d v \not u$ 
Demonstraremos a seguir a conjectura proposta por Rieffel para o caso em que a $C^{*}$-álgebra é o conjunto dos números complexos.

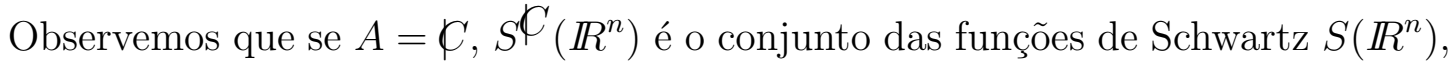
a norma $\|\cdot\|_{2}$ é a norma de $L^{2}\left(\mathbb{R}^{n}\right)$ e $\overline{S\left(\mathbb{R}^{n}\right)}=L^{2}\left(\mathbb{R}^{n}\right)$.

Notação 1.9. $\mathcal{H}=L^{2}\left(\mathbb{R}^{n}\right)$.

Teorema 1.10. . Dadas $A \in B(\mathcal{H})$ e $J$, matriz $n \times n$ anti-simétrica, $A$ é Heisenbergsuave e $\left[A, R_{G}\right]=0 \forall G \in C B^{\infty}\left(\mathbb{R}^{n}\right)$ se e somente se existe $F \in C B^{\infty}\left(\mathbb{R}^{n}\right)$ tal que $A=L_{F}$.

Cordes fez uma caracterização dos operadores suaves pela ação do grupo de Heisenberg $([\mathbf{1 7}]): A \in B(\mathcal{H})$ é Heisenberg-suave se, e somente se, existe uma função $a \in$ $C B^{\infty}\left(\mathbb{R}^{2 n}\right)$ tal que $A u(x)=\int e^{i(x-y) \xi} a(x, \xi) u(y) d y \not l \xi$, onde $\not y=(2 \pi)^{-n / 2} d y$ e $u \in$ $C_{0}^{\infty}\left(\mathbb{R}^{n}\right)$. A função $a$ é chamada de símbolo de $A$ e o operador $A$ é um operador pseudodiferencial. Ressaltamos que a integral acima é uma integral oscilatória. Além disso, prova-se facilmente que $A u \in S\left(\mathbb{R}^{n}\right)$. O teorema de Calderón-Vaillancourt [10] mostra que $A$ pode ser estendido a um operador em $\mathcal{B}(\mathcal{H}),[\mathbf{2}$, Capítulo 8 , secção 1].

Pelo visto na observação 1.8, é fácil ver que o operador $L_{F}$, onde $F \in C B^{\infty}\left(\mathbb{R}^{n}\right)$, é um operador pseudo-diferencial com símbolo $\tilde{F} \in C B^{\infty}\left(\mathbb{R}^{2 n}\right) \operatorname{com} \tilde{F}(x, \xi)=F(x-J \xi)$.

Vejamos agora um resultado que estabelece uma relação entre um operador pseudodiferencial e seu símbolo.

Observemos primeiramente que podemos ver $S\left(\mathbb{R}^{n}\right)$ como um sub-conjunto de $S^{\prime}\left(\mathbb{R}^{n}\right)$, o conjunto das distribuições temperadas, como segue: cada $f \in S\left(\mathbb{R}^{n}\right)$ determina um funcional linear contínuo em $S\left(\mathbb{R}^{n}\right), F$, dado por:

$$
<F, g>=\int f g \text { para } g \in S\left(\mathbb{R}^{n}\right)
$$

$([\mathbf{3}$, cap.8,sec.5(i) $])$.

Definição 1.11. Uma função $f \in C\left(\mathbb{R}^{n}\right)$ é dita de crescimento polinomial se existe $N \geq 0$ tal que $(1+|x|)^{-N} f$ é limitada. 
Proposição 1.12. Existe um isomorfismo $T$ em $S^{\prime}\left(\mathbb{R}^{2 n}\right)$ tal que todo $A \in \mathcal{B}(\mathcal{H})$, opera -dor pseudo-diferencial com símbolo $a \in C B^{\infty}\left(\mathbb{R}^{2 n}\right)$, satisfaz

$$
<T(a), u \otimes v>=<A v, u>\quad u, v \in S\left(\mathbb{R}^{n}\right),
$$

onde $u \otimes v$ denota a função $u \otimes v(x, y)=u(x) v(y)$.

Demonstração. Sejam $\varphi, \psi: S\left(\mathbb{R}^{2 n}\right) \rightarrow S\left(\mathbb{R}^{2 n}\right)$ dadas por

$$
\begin{gathered}
\varphi(f)(x, y)=f(x, x-y) \\
\psi(f)(x, y)=(2 \pi)^{-n} \int e^{i y \xi} f(x, \xi) d \xi .
\end{gathered}
$$

$\varphi, \psi$ são isomorfismos.

Seja $T=\varphi \circ \psi$.

$$
\begin{gathered}
<T(f), g>=\int \varphi \circ \psi(f)(x, y) g(x, y) d x d y= \\
=\int \psi(f)(x, x-y) g(x, y) d x d y=\int \psi(f)(x, z) g(x, x-z) d x d z= \\
=\int(2 \pi)^{-n}\left(\int e^{i z \cdot \xi} f(x, \xi) d \xi\right) \varphi(g)(x, z) d x d z= \\
=\int f(x, \xi)\left((2 \pi)^{-n} \int e^{i z \xi} \varphi(g)(x, z) d z\right) d x d \xi= \\
=\int f(x, \xi) \psi \circ \varphi(g)(x, \xi) d x d \xi=<f, \psi \circ \varphi(g)>.
\end{gathered}
$$

Se $\tilde{T}=\psi \circ \varphi$, temos $<T(f), g>=<f, \tilde{T}(g)>$. Então, $T$ se estende a um isomorfismo em $S^{\prime}\left(\mathbb{R}^{2 n}\right)$ (ver $[\mathbf{3}$, cap.8,sec.5]), que também chamamos $T$, definido como segue. Para $Q \in S^{\prime}\left(\mathbb{R}^{2 n}\right)$,

$$
<T(Q), g>=<Q, \tilde{T}(g)>
$$

Notemos que $T$ é um operador contínuo em $S^{\prime}\left(\mathbb{R}^{2 n}\right)$ com a topologia fraca *

Dada $a \in C B^{\infty}\left(\mathbb{R}^{2 n}\right)$, a tem crescimento polinomial; logo a define uma distribuição em $S^{\prime}\left(\mathbb{R}^{2 n}\right)([4, \mathrm{~V} .3])$. 
Para $u, v \in S\left(\mathbb{R}^{n}\right)$,

$$
<T(a), u \otimes v>=<a, \tilde{T}(u \otimes v)>
$$

$\mathrm{E}$

$$
\begin{gathered}
\tilde{T}(u \otimes v)(x, y)=\psi \circ \varphi(u \otimes v)(x, y)=(2 \pi)^{-n} \int e^{i y \xi} \varphi(u \otimes v)(x, \xi) d \xi= \\
=(2 \pi)^{-n} \int e^{i y \xi}(u \otimes v)(x, x-\xi) d \xi=(2 \pi)^{-n} \int e^{i y(x-\xi)}(u \otimes v)(x, \xi) d \xi= \\
=(2 \pi)^{-n} e^{i y x} u(x) \int e^{-i y \xi} v(\xi) d \xi
\end{gathered}
$$

Então

$$
\begin{gathered}
<a, \tilde{T}(u \otimes v)>=\int a(x, y)(2 \pi)^{-n} e^{i y x} u(x) \int e^{-i y \xi} v(\xi) d \xi d x d y= \\
=\int\left(\int e^{i y(x-\xi)} a(x, y) v(\xi) d \xi d y\right) u(x) d x=<A v, u>
\end{gathered}
$$

Corolário 1.13. Dado $A \in \mathcal{B}(\mathcal{H})$, operador pseudo-diferencial com símbolo $a \in C B^{\infty}\left(\mathbb{R}^{n}\right)$, a aplicação $a \rightarrow A$ é injetora.

Demonstração. Pela proposição (1.12), temos que existe um isomorfismo $T$ em $S^{\prime}\left(\mathbb{R}^{2 n}\right)$ tal que, para $u, v \in S\left(\mathbb{R}^{n}\right)$ vale $\langle T(a), u \otimes v>=<A v, u>$. Logo, se $A=0$, $<T(a), u \otimes v>=0$. Como $S\left(\mathbb{R}^{n}\right) \otimes S\left(\mathbb{R}^{n}\right)$ é denso em $S\left(\mathbb{R}^{2 n}\right)$, [8, teorema 51.6], então temos que $T(a)=0$, o que implica $a=0$.

Nota 1.14. Citaremos a seguir, na proposição (1.16), o lema 2 de [14], que afirma que existe uma seqüência $\left(e_{k}\right)_{k \in \mathbb{N}}$ em $S\left(\mathbb{R}^{n}\right)$ tal que $L_{e_{k}}$ converge para a identidade na topologia forte de operadores.

Notação 1.15. Seja $\mathcal{A}$ o conjunto dos operadores Heisenberg-suaves, $A$, tais que

$$
\left[A, R_{G}\right]=0 \quad \forall G \in C B^{\infty}\left(\mathbb{R}^{n}\right)
$$

Pela caracterização de Cordes, todo $A \in \mathcal{A}$ é da forma $A=a(x, D)$ para alguma $a \in C B^{\infty}\left(\mathbb{R}^{2 n}\right)$.

Proposição 1.16. Dado $A \in \mathcal{A}$, existe uma seqüência $\left(f_{k}\right)_{k \in \mathbb{N}}$ em $S\left(\mathbb{R}^{n}\right)$ de modo que $L_{f_{k}}$ convege para $A$ na topologia forte de operadores. 
Demonstração. Seja $\left(e_{k}\right)_{k \in \mathbb{N}}$ uma seqüência em $S\left(\mathbb{R}^{n}\right)$ tal que $L_{e_{k}}$ converge para a identidade na topologia forte de operadores $\left[\mathbf{1 4}\right.$, Lema 2]. Como $A$ mantém $S\left(\mathbb{R}^{n}\right)$ invariante, $A\left(e_{k}\right) \in S\left(\mathbb{R}^{n}\right)$. Seja $f_{k}=A\left(e_{k}\right)$.

Dada $u \in S\left(\mathbb{R}^{n}\right)$,

$$
L_{f_{k}} u=f_{k} \times_{J} u=A e_{k} \times_{J} u=R_{u} A e_{k}=A R_{u} e_{k}=A L_{e_{k}} u .
$$

Assim, $L_{f_{k}}=A L_{e_{k}}$, pois $S\left(\mathbb{R}^{n}\right)$ é denso em $\mathcal{H}$; e, como $L_{e_{k}}$ converge para $I \in \mathcal{B}(\mathcal{H})$ na topologia forte de operadores, então $L_{f_{k}}$ converge para $A$ nesta topologia.

Proposição 1.17. Dado $A \in \mathcal{A}$, seja $\left(F_{k}\right)$ uma seqüência de funções em $C B^{\infty}\left(\mathbb{R}^{n}\right)$ de modo que $A$ é limite de $L_{F_{k}}$ na topologia forte de operadores. Se $a$ é o símbolo de $A$ e $a_{k}$ é o símbolo de $L_{F_{k}}$, então temos $a_{k} \longrightarrow a \mathrm{em} S^{\prime}\left(\mathbb{R}^{2 n}\right)$.

Demonstração. Por hipótese vale que

$$
L_{F_{k}} v \longrightarrow A v \quad \forall v \in H
$$

Logo,

$$
<L_{F_{k}} v, u>\longrightarrow<A v, u>\quad \forall u, v \in S\left(\mathbb{R}^{n}\right) .
$$

Logo, pela proposição (1.12)

$$
<T\left(a_{k}\right), u \otimes v>\longrightarrow<T(a), u \otimes v>
$$

ou seja, $\left\langle T\left(a_{k}\right)-T(a), u \otimes v>\longrightarrow_{k \rightarrow \infty} 0\right.$.

Queremos provar que, para toda $h \in S\left(\mathbb{R}^{2 n}\right),<T\left(a_{k}\right)-T(a), h>\longrightarrow_{k \rightarrow \infty} 0$.

Primeiramente, observemos que, pelo Princípio da Limitação Uniforme, $\sup \left\|L_{F_{k}}\right\|<$ $\infty(\operatorname{ver}[6$, teorema 2.6]).

Além disso, vemos em [8, teorema 51.6] que $S\left(\mathbb{R}^{n}\right) \otimes S\left(\mathbb{R}^{n}\right)$ é denso em $S\left(\mathbb{R}^{2 n}\right)$. Pelo corolário anterior ao teorema 51.6, temos que $S\left(\mathbb{R}^{n}\right)$ é nuclear; logo, pelo feito na segunda página do capítulo 50, temos que $S\left(\mathbb{R}^{n}\right) \otimes S\left(\mathbb{R}^{n}\right)=S\left(\mathbb{R}^{n}\right) \otimes_{\pi} S\left(\mathbb{R}^{n}\right)$, onde $\otimes_{\pi}$ é definido em [8, capítulo 43, definição 43.2].

Assim, pelo teorema 45.1 de $[8]$, temos que dada $h \in S\left(\mathbb{R}^{2 n}\right)$, existem seqüências $\left(u_{j}\right)_{j \in \mathbb{N}}$ e $\left(v_{j}\right)_{j \in \mathbb{N}}$ em $S\left(\mathbb{R}^{2 n}\right)$, convergindo a zero, e $\left(\lambda_{j}\right)_{j \in \mathbb{N}}, \lambda_{j} \in \mathscr{C}$, tal que $\sum_{j}\left|\lambda_{j}\right|<$ $\infty$, que satisfazem

$$
h=\sum_{j} \lambda_{j} u_{j} \otimes v_{j}
$$


Como visto acima, temos que, para todo $j$,

$$
\lim _{k \rightarrow \infty}<T\left(a_{k}\right)-T(a), u_{j} \otimes v_{j}>=0
$$

E, considerando que:

1. $<T\left(a_{k}\right)-T(a), u_{j} \otimes v_{j}>=<\left(L_{F_{k}}-A\right) v_{j}, u_{j}>$,

2. $\left(u_{j}\right)$ e $\left(v_{j}\right)$ convergem a zero em $S\left(\mathbb{R}^{n}\right)$ e, portanto, convergem a zero em $L^{2}\left(\mathbb{R}^{n}\right)$,

3. $\sup _{k}\left\|L_{F_{k}}\right\|<\infty$,

temos que $\sup _{k, j}\left|<T\left(a_{k}\right)-T(a), u_{j} \otimes v_{j}>\right|<\infty$. Portanto, pelo Teorema da Convergência Dominada para séries, $\lim _{k \rightarrow \infty} \sum_{j} \lambda_{j}<T\left(a_{k}\right)-T(a), u_{j} \otimes v_{j}>=0$.

Isto é, $\lim _{k \rightarrow \infty}<T\left(a_{k}\right)-T(a), h>=0$. Como $T$ é isomorfismo em $S^{\prime}\left(\mathbb{R}^{2 n}\right)$, temos então que $\left(a_{k}\right)$ converge para $a \mathrm{em} S^{\prime}\left(\mathbb{R}^{2 n}\right)$.

Definição 1.18. Dadas funções $a, b \in C^{\infty}\left(\mathbb{R}^{2 n}\right)$, definimos seu colchete de Poisson como segue:

$$
\{a, b\}=\sum_{j=1}^{n}\left(\frac{\partial a}{\partial x_{j}} \frac{\partial b}{\partial \xi_{j}}-\frac{\partial a}{\partial \xi_{j}} \frac{\partial b}{\partial x_{j}}\right) .
$$

Proposição 1.19. Dadas $F, G \in C B^{\infty}\left(\mathbb{R}^{n}\right)$ e $J$ matriz anti-simétrica $n \times n$, sejam

$$
a(x, \xi)=F(x-J \xi)
$$

e

$$
b(x, \xi)=G(x+J \xi)
$$

Então, $\{a, b\}=0$.

Demonstração. Fazendo contas, temos que:

$$
\begin{array}{cl}
\frac{\partial a}{\partial x_{i}}=\partial_{i} F & \frac{\partial a}{\partial \xi_{i}}=\sum_{j=1}^{n} J_{i j} \partial_{j} F \\
\frac{\partial b}{\partial x_{i}}=\partial_{i} G & \frac{\partial b}{\partial \xi_{i}}=-\sum_{j=1}^{n} J_{i j} \partial_{j} G
\end{array}
$$


$\frac{\partial a}{\partial x_{i}} \frac{\partial b}{\partial \xi_{i}}-\frac{\partial a}{\partial \xi_{i}} \frac{\partial b}{\partial x_{i}}=-\partial_{i} F \sum_{j=1}^{n} J_{i j} \partial_{j} G-\partial_{i} G \sum_{j=1}^{n} J_{i j} \partial_{j} F=\partial_{i} F \sum_{j=1}^{n} J_{j i} \partial_{j} G+\partial_{i} G \sum_{j=1}^{n} J_{j i} \partial_{j} F$.

Assim,

$$
\{a, b\}=\sum_{j=1}^{n} \sum_{j=1}^{n}\left(J_{j i} \partial_{i} F \partial_{j} G+J_{j i} \partial_{i} G \partial_{j} F\right)
$$

e $\{a, b\}=0$ pois $J$ é anti-simétrica.

Notação 1.20. Seja $\mathcal{P}_{B}\left(\mathbb{R}^{n}\right)$ o conjunto das funções em $C^{\infty}\left(\mathbb{R}^{n}\right)$ tais que elas e todas as suas derivadas são de crescimento polinomial.

Proposição 1.21. Dadas $A \in \mathcal{A}$, com símbolo $a \in C B^{\infty}\left(\mathbb{R}^{n}\right)$, e a função $b(x, \xi)=$ $G(x+J \xi), \operatorname{com} G \in \mathcal{P}_{B}\left(\mathbb{R}^{2 n}\right)$, então $\{a, b\}=0$.

Demonstração. Dado $A \in \mathcal{A}$, existe uma seqüência $\left(A_{k}\right)_{k \in \mathbb{N}}$, onde $A_{k}=L_{F_{k}}$, com $F_{k} \in S\left(\mathbb{R}^{n}\right)$, de modo que $\left(A_{k}\right)_{k \in \mathbb{N}}$ converge para $A$ na topologia forte de operadores, 1.16. Se $a_{k}$ é o símbolo de $A_{k}$, vimos que $\left(a_{k}\right)_{k \in \mathbb{N}}$ converge para $a$ em $S^{\prime}\left(\mathbb{R}^{2 n}\right)(1.17)$. Como o símbolo de $A_{k}$ é $F_{k}(x-J \xi)$, temos pela proposição anterior que $\left\{a_{k}, b\right\}=$ $0, \quad \forall k$.

Como $b \in \mathcal{P}_{B}\left(\mathbb{R}^{2 n}\right),\left\{a_{k}, b\right\} \in S^{\prime}\left(\mathbb{R}^{2 n}\right), \forall k$. Como $a_{k} \longrightarrow a$ em $S^{\prime}\left(\mathbb{R}^{2 n}\right)$, e como a multiplicação por uma derivada qualquer da função $b$ é uma operação contínua em $S^{\prime}\left(\mathbb{R}^{2 n}\right)([4, \mathrm{~V} .3])$, temos que :

$$
\frac{\partial b}{\partial \xi_{i}} \frac{\partial a_{k}}{\partial x_{i}} \longrightarrow \frac{\partial b}{\partial \xi_{i}} \frac{\partial a}{\partial x_{i}}
$$

e

$$
\frac{\partial b}{\partial x_{i}} \frac{\partial a_{k}}{\partial \xi_{i}} \longrightarrow \frac{\partial b}{\partial x_{i}} \frac{\partial a}{\partial \xi_{i}}
$$

em $S^{\prime}\left(\mathbb{R}^{2 n}\right)$, e vale que $\left\{a_{k}, b\right\} \longrightarrow\{a, b\}$ em $S^{\prime}\left(\mathbb{R}^{2 n}\right)$. Como $\left\{a_{k}, b\right\}=0 \quad \forall k$ temos que $\{a, b\}=0$.

Lema 1.22. Seja $a \in C B^{\infty}\left(\mathbb{R}^{2 n}\right)$ que satisfaz $\{a, b\}=0$ para qualquer $b \in \mathcal{P}_{B}\left(\mathbb{R}^{2 n}\right)$ $\operatorname{com} b(x, \xi)=G(x+J \xi)$. Então existe $F \in C B^{\infty}\left(\mathbb{R}^{n}\right)$ tal que $a(x, \xi)=F(x-J \xi)$. 
Demonstração. Seja $b_{i}$ a projeção de $x+J \xi$ na coordenada $i$,

$$
b_{i}(x, \xi)=x_{i}+\sum_{k=1}^{n} J_{i k} \xi_{k}
$$

Assim definida, $b_{i} \in \mathcal{P}_{B}\left(\mathbb{R}^{2 n}\right)$ e, pela proposição anterior, temos $\left\{a, b_{i}\right\}=0$, para todo $i=1, \cdots, n$. Logo,

$$
\sum_{j=1}^{n}\left(\frac{\partial a}{\partial x_{j}} J_{i j}-\frac{\partial a}{\partial \xi_{j}} \delta_{i j}\right)=\sum_{j=1}^{n} J_{i j} \frac{\partial a}{\partial x_{j}}-\frac{\partial a}{\partial \xi_{i}}=0
$$

Ou seja,

$$
\frac{\partial a}{\partial \xi_{i}}=\sum_{j=1}^{n} J_{i j} \frac{\partial a}{\partial x_{j}} \quad i=1, \cdots, n .
$$

Portanto, aplicando as idéias de [16, 2.1], temos que

$$
a(x, \xi)=a\left((x, \xi)+s\left(-J_{i 1}, \cdots,-J_{i n}, e_{i}\right)\right),
$$

para todo $(x, \xi) \in \mathbb{R}^{2 n}$, para todo $s \in \mathbb{R}$, para qualquer $i \in\{1,2, \cdots, n\}$, onde $\left\{e_{i}\right\}$ é uma base ortonormal de $\mathbb{R}^{n}, e_{i}=(0, \cdots, 0,1,0, \cdots, 0)$.

Agora, em $(* *)$, consideremos $s_{1}$ em lugar de $s, i=1$, e $(x . \xi)=\left(x_{0}, 0\right)$ :

$a\left(x_{0}, 0\right)=a\left(\left(x_{0}, 0\right)+s_{1}\left(-J_{11}, \cdots,-J_{1 n}, e_{1}\right)\right)=a\left(\left(x_{0}, 0\right)+\left(-J_{11} s_{1}, \cdots,-J_{1 n} s_{1}, s_{1} e_{1}\right)\right)$.

Apliquemos $(* *)$ novamente para $s_{2}$ no lugar de $s, i=2$ e $(x, \xi)=\left(x_{0}-\left(J_{11} s_{1}, \cdots, J_{1 n} s_{1}\right)\right.$, $\left.s_{1} e_{1}\right)$. Assim, temos

$$
a\left(x_{0}, 0\right)=a\left(x_{0}+\left(-J_{11} s_{1}, \cdots,-J_{1 n} s_{1}\right)+\left(-J_{21} s_{2}, \cdots,-J_{2 n} s_{2}\right), s_{1} e_{1}+s_{2} e_{2}\right) .
$$

Repetindo o raciocínio para $i=3, \cdots, n$, temos

$$
a\left(x_{0}, 0\right)=a\left(x_{0}-\left(\sum_{i=1}^{n} J_{i 1} s_{i}, \cdots, \sum_{i=1}^{n} J_{i n} s_{n}\right), \sum_{i=1}^{n} s_{i} e_{i}\right) .
$$

Como $J$ é anti-simétrica, tomando $\xi=\left(s_{1}, \cdots, s_{n}\right)$, temos:

$$
a\left(x_{0}, 0\right)=a\left(x_{0}+J \xi, \xi\right) .
$$

Seja $F(x)=a(x, 0)$. Então $a(x, \xi)=F(x-J \xi)$. 
Corolário 1.23. Dado $A \in \mathcal{A}$, existe $F \in C B^{\infty}\left(\mathbb{R}^{n}\right)$ tal que $A=L_{F}$.

Demonstração. Dado $A \in \mathcal{A}$, pela caracterização feita por Cordes dos operadores Heisenberg-suaves, $A$ é um operador pseudo-diferencial. Seja $a \in C B^{\infty}\left(\mathbb{R}^{2 n}\right)$ seu símbolo. Pela proposição (1.21), dada $b(x, \xi)=G(x+J \xi)$, com $G \in \mathcal{P}_{B}\left(\mathbb{R}^{n}\right)$, temos $\{a, b\}=0$. Assim, pelo lema (1.22), existe $F \in C B^{\infty}\left(\mathbb{R}^{n}\right)$ tal que $a(x . \xi)=F(x-J \xi)$. Temos, então, que o operador $A$ tem símbolo $F(x-J \xi)$, ou seja, $A=L_{F}$.

Com isto fica provado o Teorema (1.10) e portanto a questão proposta por Rieffel, no caso da $C^{*}$-álgebra ser o conjunto dos números complexos. 


\section{Capítulo 2}

Seja $A$ uma $C^{*}$-álgebra separável ao longo deste capítulo. Consideremos, novamente, a ação do grupo de Heisenberg em $\mathcal{B}^{*}\left(\overline{S^{A}\left(\mathbb{R}^{n}\right)}\right)$,

$$
(z, \zeta) \rightarrow E_{z, \zeta}^{-1} T E_{z, \zeta}
$$

onde $T \in \mathcal{B}^{*}\left(\overline{S^{A}\left(\mathbb{R}^{n}\right)}\right)$ e $E_{z, \zeta} u(x)=e^{i \zeta x} u(x-z)$ se $u \in S^{A}\left(\mathbb{R}^{n}\right)$.

Notação 2.1. $T_{z, \zeta}=E_{z, \zeta}^{-1} T E_{z, \zeta}$.

Definição 2.2. Dizemos que $T \in \mathcal{B}^{*}\left(\overline{S^{A}\left(\mathbb{R}^{n}\right)}\right)$ é Heisenberg-suave se a aplicação $(z, \zeta) \mapsto$ $T_{z, \zeta}$ é $C^{\infty}$. Dizemos que $T \in \mathcal{B}^{*}\left(\overline{S^{A}\left(\mathbb{R}^{n}\right)}\right)$ é suave por translações se a aplicação $z \mapsto T_{z, 0}$ é $C^{\infty}$.

Neste capítulo veremos que dado um operador $T$ em $\mathcal{B}^{*}\left(\overline{S^{A}\left(\mathbb{R}^{n}\right)}\right)$ que comuta com qualquer operador $R_{g}$ para $g$ em $S^{A}\left(\mathbb{R}^{n}\right)$ (ver capítulo 1 ), se $T$ é suave por translações então é Heisenberg-suave.

Lema 2.3. Seja $f: \mathbb{R}^{n} \rightarrow A$ uma função contínua tal que $\sup _{x \in \mathbb{R}^{n}}|x|^{l}\|f(x)\|<\infty$ para todo inteiro positivo $l$. Então, dada $J$, matriz anti-simétrica $n \times n$, para cada $l \in \mathbb{Z}^{+}$e para todo $\varepsilon>0$, existe $\delta>0$ de modo que

$$
|x|^{l}\left\|f(x+J \xi) e^{i x \xi}-f(x)\right\|<\varepsilon
$$

para quaisquer $x, \xi \in \mathbb{R}^{n} \operatorname{com}|\xi|<\delta$. 
Demonstração. Dados $\varepsilon>0, l \in \mathbb{Z}^{+}$, existe $R_{l} \in \mathbb{N}$ tal que se $|x|>R_{l}$,

$$
|x|^{l}\|f(x)\|<\frac{\varepsilon}{2} .
$$

Seja $d_{J}, 0<d_{J} \leq 1$, tal que se $|\xi|<d_{J},|J \xi|<1$. Consideremos

$$
X_{f, l}=\left\{(x, \xi) \in \mathbb{R}^{2 n},|x| \leq R_{l}+1,|\xi| \leq d_{J}\right\}
$$

Embora a notação fique um pouco "carregada", consideramos que é necessário para situar mais facilmente o leitor.

$\underline{1^{o} \text { passo: }} l=0$.

Se $g(x, \xi)=f(x+J \xi) e^{i x \xi}, g$ é uniformemente contínua em $X_{f, 0}$, pois $X_{f, 0}$ é compacto. Logo, dado $\varepsilon>0$ existe $\delta>0, \delta \leq d_{J}$, tal que se $\left|(x, \xi)-\left(x^{\prime}, \xi^{\prime}\right)\right|<\delta$ e $(x, \xi),\left(x^{\prime}, \xi^{\prime}\right) \in X_{f, 0}$, temos $\left\|g(x, \xi)-g\left(x^{\prime}, \xi^{\prime}\right)\right\|<\varepsilon$. Seja, agora, $\left(x^{\prime}, \xi^{\prime}\right)=(x, 0)$. Assim $\left|(x, \xi)-\left(x^{\prime}, \xi^{\prime}\right)\right|=|\xi|$. Portanto, se $|\xi|<\delta,\left\|f(x+J \xi) e^{i x \xi}-f(x)\right\|<\varepsilon$ para todo $x \in \mathbb{R}^{n}$ $\operatorname{com}|x| \leq R_{0}+1$.

Se $|x|>R_{0}+1$ e $|\xi|<\delta,|x+J \xi|>R_{0}$ pois $|J \xi|<1$, e temos, por (1):

$$
\left\|f(x+J \xi) e^{i x \xi}-f(x)\right\| \leq\|f(x+J \xi)\|+\|f(x)\|<\frac{\varepsilon}{2}+\frac{\varepsilon}{2}=\varepsilon .
$$

20 passo: $l$ qualquer.

Seja $h(x)=|x|^{l} f(x)$. Pelo primeiro passo, aplicado a $h$, temos que existe $\sigma_{l} \leq d_{J}$ tal que, se $|\xi|<\sigma_{l}$, então

$$
\left\||x+J \xi|^{l} f(x+J \xi) e^{i x \xi}-|x|^{l} f(x)\right\|<\varepsilon .
$$

E notemos que, por $(2)$, se $|\xi|<\sigma_{l}$,

$$
\begin{gathered}
|x|^{l}\left\|f(x+J \xi) e^{i x \xi}-f(x)\right\| \leq\left\||x+J \xi|^{l} f(x+J \xi) e^{i x \xi}-|x|^{l} f(x)\right\|+\||x|^{l} f(x+J \xi) e^{i x \xi}- \\
|x+J \xi|^{l} f(x+J \xi) e^{i x \xi}\left\|<\varepsilon+\left.|| x\right|^{l}-|x+J \xi|^{l} \mid\right\| f(x+J \xi) \| \\
\text { Se }(x, \xi) \in X_{f, l}, \quad|x+J \xi| \leq R_{l}+2, \text { pois }|J \xi|<1 .
\end{gathered}
$$

Seja $K=\sup _{|y| \leq R_{l}+2}\|f(y)\|$. Se $p(x, \xi)=|x+J \xi|^{l}, p$ é uniformemente contínua em $X_{f, l}$. Assim, dado $\varepsilon>0$, existe $\delta_{l}>0, \delta_{l} \leq \sigma_{l}$ ( $\delta_{l}$ depende de $l$ ), tal que se 
$(x, \xi),\left(x^{\prime}, \xi^{\prime}\right) \in X_{f, l}$ e $\left|(x, \xi)-\left(x^{\prime}, \xi^{\prime}\right)\right|<\delta_{l}$ então $\left|p(x, \xi)-p\left(x^{\prime}, \xi^{\prime}\right)\right|<\frac{\varepsilon}{K}$. Tomando $\left(x^{\prime}, \xi^{\prime}\right)=(x, 0)$, temos ||$x+\left.J \xi\right|^{l}-|x|^{l} \mid<\frac{\varepsilon}{K}$ para $|\xi|<\delta_{l}$ e $|x| \leq R_{l}+1$.

Logo, se $|\xi|<\delta_{l}$, temos $|x|^{l}\left\|f(x+J \xi) e^{i x \xi}-f(x)\right\|<2 \varepsilon$ para $|x| \leq R_{l}+1$.

Se $|x|>R_{l}+1$, e $|\xi|<\delta_{l} \leq \sigma_{l} \leq d_{J}$, temos que $|x+J \xi|>R_{l}$ pois $|J \xi|<1$. Sem perda de generalidade, consideremos $R_{l}>1$ e teremos: $|J \xi|<1<R_{l}<|x+J \xi|$.

Assim

$$
|x|=|x+J \xi-J \xi| \leq|x+J \xi|+|J \xi| \leq 2|x+J \xi| .
$$

O lado direito de (3) é, portanto, menor ou igual a

$$
\begin{gathered}
\varepsilon+|x|^{l}\|f(x+J \xi)\|+|x+J \xi|^{l}\|f(x+J \xi)\| \leq \\
\leq \varepsilon+2^{l}|x+J \xi|^{l}\|f(x+J \xi)\|+|x+J \xi|^{l}\|f(x+J \xi)\|<\varepsilon+\left(2^{l}+1\right) \frac{\varepsilon}{2},
\end{gathered}
$$

por (1), já que $|x+J \xi|>R_{l}$.

Ou seja, dado $\varepsilon>0$, existe $\delta_{l}>0$ tal que

$$
|x|^{l}\|f(x+J \xi)\| e^{i x \xi}-f(x) \|<\varepsilon\left(2^{l-1}+\frac{3}{2}\right)
$$

para $x, \xi \in \mathbb{R}^{n} \operatorname{com}|x|>R_{l}+1$ e $|\xi|<\delta_{l}$.

Para todo $\varepsilon^{\prime}>0$, seja $\varepsilon>0$ de modo que $\varepsilon\left(2^{l-1}+\frac{3}{2}\right)<\varepsilon^{\prime}$, e $2 \varepsilon<\varepsilon^{\prime}$.

Assim, temos que, para qualquer $\varepsilon^{\prime}>0$, existe $\delta_{l}>0$ tal que $|x|^{l} \| f(x+J \xi) e^{i x \xi}-$ $f(x) \|<\varepsilon^{\prime}$, para todo $x \in \mathbb{R}^{n}$ e $\xi \in \mathbb{R}^{n}$ com $|\xi|<\delta_{l}$, tendo fixado $l \in \mathbb{N}$.

Observação 2.4. Usaremos o lema (2.3) na demonstração da proposição (2.5) do seguinte modo: estaremos considerando a função $s(x)=(1+|x|)^{n}$ e vamos provar que, dado $\varepsilon_{1}>0$, existe $\delta>0$ tal que, se $|\xi|<\delta, s(x)\left\|f(x+J \xi) e^{i x \xi}-f(x)\right\|<\varepsilon_{1}$. Notemos que $s(x)$ é uma soma finita de termos do tipo $c_{l}|x|^{l}$, onde $c_{l} \in \mathbb{Z}^{+}$é uma constante, e $l \in\{0,1, \cdots, n\}$. Dado qualquer $\varepsilon_{1}>0$, escolhemos $\varepsilon>0$ de modo que $\sum c_{l} \varepsilon<\varepsilon_{1}$. Para cada valor de $l$, aplicamos o lema (2.3). Isto demonstra a afirmação.

Proposição 2.5. Dada $J$, matriz anti-simétrica $n \times n$, existe uma seqüência $\left(e_{k}\right)$ em $S^{A}\left(\mathbb{R}^{n}\right)$ tal que, para toda $f \in S^{A}\left(\mathbb{R}^{n}\right)$, temos $\lim _{k \rightarrow 0} L_{e_{k}}(f)=f$ em $S^{A}\left(\mathbb{R}^{n}\right)$ com \|\|$_{2}$. 
Demonstração. Como $A$ é separável, admite uma aproximação da unidade que é uma seqüência $([\boldsymbol{5}, \operatorname{Remark}(3.11)])$. Seja $\left(u_{k}\right)_{k \in \mathbb{N}}$ uma aproximação da unidade para $A$, com $u_{k} \geq 0$ e $\left\|u_{k}\right\| \leq 1$ para todo $k \in \mathbb{N}$.

Seja $\psi \in C_{c}^{\infty}\left(\mathbb{R}^{n}\right), \psi \geq 0$ tal que o suporte de $\psi$ está contido na bola unitária, $(\operatorname{supp}(\psi) \subseteq B(0,1))$, e $\int_{\mathbb{R}^{n}} \psi(\xi) d \xi=1$. Para $k \in \mathbb{Z}^{+}$, seja $\psi_{k}(\xi)=k^{n} \psi(k \xi) ;$ assim $\operatorname{supp}\left(\psi_{k}\right) \subseteq B\left(0, \frac{1}{k}\right)$. Seja $\phi_{k}=\psi_{k} u_{k}$. Assim $\operatorname{supp}\left(\phi_{k}\right) \subseteq B\left(0, \frac{1}{k}\right)$ e $\int_{\mathbb{R}^{n}} \phi_{k}(\xi) d \xi=u_{k}$. Seja $\varphi_{k}$ dada por $\int_{\mathbb{R}^{n}} e^{-i y \xi} \varphi_{k}(y) d y=\phi_{k}(\xi)$. Como $\phi_{k} \in C_{c}^{\infty}\left(\mathbb{R}^{n}, A\right), \varphi_{k} \in S^{A}\left(\mathbb{R}^{n}\right)$. Como feito na proposição, 1.1, e, por $[\mathbf{1},(1.13)]$, para $f \in S^{A}\left(\mathbb{R}^{n}\right)$ temos que $R_{f}\left(\varphi_{k}\right)(x)=$ $\int e^{i x \xi} \phi_{k}(\xi) f(x+J \xi) d \xi$.

Como $\int \phi_{k}(\xi) f(x) d \xi=u_{k} f(x)$, temos:

$$
\begin{gathered}
\left\|(2 \pi)^{\frac{n}{2}} R_{f}\left(\varphi_{k}\right)(x)-u_{k} f(x)\right\| \leq \int\left\|e^{i x \xi} \phi_{k}(\xi) f(x+J \xi)-\phi_{k}(\xi) f(x)\right\| d \xi \leq \\
\leq \int\left\|\phi_{k}(\xi)\right\|\left\|e^{i x \xi} f(x+J \xi)-f(x)\right\| d \xi
\end{gathered}
$$

Pela observação (2.4) temos que, para todo $\varepsilon>0$ existe $\delta>0$ tal que, se $k>\frac{1}{\delta}$ :

$$
s(x)\left\|(2 \pi)^{\frac{n}{2}} R_{f}\left(\varphi_{k}\right)(x)-u_{k} f(x)\right\| \leq \varepsilon \int_{B\left(0, \frac{1}{k}\right)}\left\|\phi_{k}(\xi)\right\| d \xi<\varepsilon,
$$

pois

$$
\int_{B\left(0, \frac{1}{k}\right)}\left\|\phi_{k}(\xi)\right\| d \xi=\int_{B\left(0, \frac{1}{k}\right)} \psi_{k}(\xi) d \xi\left\|u_{k}\right\| \leq 1, \text { para } k>\frac{1}{\delta} .
$$

Portanto

$$
\left\|(2 \pi)^{\frac{n}{2}} R_{f}\left(\varphi_{k}\right)-u_{k} f\right\|_{2}^{2} \leq \int_{\mathbb{R}^{n}}\left\|(2 \pi)^{\frac{n}{2}} R_{f}\left(\varphi_{k}\right)(x)-u_{k} f(x)\right\|^{2} d x \leq \varepsilon^{2} \int_{\mathbb{R}^{n}} \frac{1}{s(x)^{2}} d x .
$$

Por outro lado, $\left\|f-u_{k} f\right\|_{2}^{2} \leq \int\left\|\left(f-u_{k} f\right)(x)\right\|^{2} d x$. Como $u_{k}$ é aproximação da unidade em $A$, para cada $x \in \mathbb{R}^{n}$ temos que $\lim _{k \rightarrow \infty}\left\|f(x)-u_{k} f(x)\right\|=0$. Além disso, $\left\|f(x)-u_{k} f(x)\right\| \leq 2\|f(x)\|$. Como $2\|f(x)\| \in L^{1}\left(\mathbb{R}^{n}\right)$, pelo Teorema da Convergência Dominada, temos que $\lim _{k \rightarrow \infty} \int\left\|\left(f-u_{k} f\right)(x)\right\|^{2} d x=0$, o que implica que

$$
\lim _{k \rightarrow \infty}\left\|f-u_{k} f\right\|_{2}=0 .
$$


Portanto, por (1) e (2), $\lim _{k \rightarrow \infty}\left\|(2 \pi)^{\frac{n}{2}} R_{f}\left(\varphi_{k}\right)-f\right\|_{2}=0$.

Tomando $e_{k}=(2 \pi)^{-\frac{n}{2}} \varphi_{k}$, temos que $\lim _{k \rightarrow \infty}\left\|R_{f}\left(e_{k}\right)-f\right\|_{2}=0$.

Observamos que $R_{f}\left(e_{k}\right)=e_{k} \times_{J} f=L_{e_{k}}(f)$. Portanto, temos que para toda $f \in$ $S^{A}\left(\mathbb{R}^{n}\right), \quad \lim _{k \rightarrow \infty} L_{e_{k}}(f)=f$ em $\left(S^{A}\left(\mathbb{R}^{n}\right),\|\cdot\|_{2}\right)$.

Usamos agora argumentos semelhantes a [9, secção 1].

Definição 2.6. Dada $F \in \overline{S^{A}\left(\mathbb{R}^{n}\right)}$, para cada $g \in S^{A}\left(\mathbb{R}^{n}\right)$ definimos $L_{F}(g)=R_{g}(F)$. Como $R_{g}$ é contínuo em $\left(S^{A}\left(\mathbb{R}^{n}\right),\|\cdot\|_{2}\right)$, se estende a $\overline{S^{A}\left(\mathbb{R}^{n}\right)}$, logo $L_{F}$ está bem definido, como operador de $S^{A}\left(\mathbb{R}^{n}\right)$ em $\overline{S^{A}\left(\mathbb{R}^{n}\right)}$.

Corolário 2.7. Dado $T \in \mathcal{B}^{*}\left(\overline{S^{A}\left(\mathbb{R}^{n}\right)}\right)$ que comuta com $R_{g}$ para qualquer $g \in S^{A}\left(\mathbb{R}^{n}\right)$, existe $\left(F_{k}\right)$ em $\overline{S^{A}\left(\mathbb{R}^{n}\right)}$ tal que $\lim _{k \rightarrow \infty} L_{F_{k}}(g)=T(g), \forall g \in S^{A}\left(\mathbb{R}^{n}\right)$.

Demonstração. Pela proposição (2.5) existe uma seqüência $\left(e_{k}\right)$ em $S^{A}\left(\mathbb{R}^{n}\right)$ tal que $\lim _{k \rightarrow \infty} L_{e_{k}}(g)=g, \forall g \in S^{A}\left(\mathbb{R}^{n}\right)$.

Por outro lado, temos que $L_{T e_{k}} g=R_{g} T e_{k}=T R_{g} e_{k}=T L_{e_{k}} g$. Portanto,

$$
\lim _{k \rightarrow \infty} L_{T_{e_{k}}}(g)=T(g), \forall g \in S^{A}\left(\mathbb{R}^{n}\right)
$$

Tomando $F_{k}=T e_{k}$ temos a tese.

Observação 2.8. Notemos que o operador $L_{F_{k}}$ acima é contínuo para todo $k \in \mathbb{Z}^{+}$, pois $\left\|L_{F_{k}}\right\|=\left\|L_{T e_{k}}\right\|=\left\|T L_{e_{k}}\right\| \leq\|T\|\left\|L_{e_{k}}\right\|$.

Proposição 2.9. Sejam $U, V \in \mathcal{B}^{*}\left(\overline{S^{A}\left(\mathbb{R}^{n}\right)}\right)$ operadores que preservam $S^{A}\left(\mathbb{R}^{n}\right)$. Suponhamos que para toda $h \in S^{A}\left(\mathbb{R}^{n}\right)$ vale $U^{*} L_{h} U=V^{*} L_{h} V$. Então, dada $F \in \overline{S^{A}\left(\mathbb{R}^{n}\right)}$, temos $U^{*} L_{F} U=V^{*} L_{F} V$.

Aqui estamos vendo os operadores $U^{*} L_{F} U$ e $V^{*} L_{F} V$ como operadores de $S^{A}\left(\mathbb{R}^{n}\right)$ em $\overline{S^{A}\left(\mathbb{R}^{n}\right)}$, não necessariamente limitados.

Demonstração. Dada $F \in \overline{S^{A}\left(\mathbb{R}^{n}\right)}$, existe uma seqüência $\left(h_{k}\right)$ em $S^{A}\left(\mathbb{R}^{n}\right)$ tal que $\lim _{k \rightarrow \infty} h_{k}=F$ em $\overline{S^{A}\left(\mathbb{R}^{n}\right)}$. Dada $g \in S^{A}\left(\mathbb{R}^{n}\right)$, como $R_{g}$ é contínuo em $\overline{S^{A}\left(\mathbb{R}^{n}\right)}$, temos

$$
L_{F}(g)=R_{g}(F)=\lim _{k \rightarrow \infty} R_{g}\left(h_{k}\right)
$$


Calculemos (lembrando que $U(g) \in S^{A}\left(\mathbb{R}^{n}\right)$ e $V(g) \in S^{A}\left(\mathbb{R}^{n}\right)$ :

$$
\begin{gathered}
U^{*} L_{F} U(g)=U^{*} \lim _{k \rightarrow \infty} R_{U(g)}\left(h_{k}\right)=\lim _{k \rightarrow \infty} U^{*} R_{U(g)}\left(h_{k}\right)= \\
=\lim _{k \rightarrow \infty} U^{*} L_{h_{k}} U(g)=\lim _{k \rightarrow \infty} V^{*} L_{h_{k}} V(g)=V^{*} \lim _{k \rightarrow \infty} R_{V(g)}\left(h_{k}\right)=V^{*} L_{F} V(g) .
\end{gathered}
$$

Lema 2.10. Dada $h \in S^{A}\left(\mathbb{R}^{n}\right)$ vale que $\left(L_{h}\right)_{z, \zeta}=\left(L_{h}\right)_{z-J \zeta, 0}$.

Demonstração. Lembremos que $\left(L_{h}\right)_{z, \zeta}=E_{z, \zeta}^{-1} L_{h} E_{z, \zeta}$, onde $E_{z, \zeta}=M_{\zeta} T_{z}$, com $M_{\zeta} u(x)=e^{i \zeta x} u(x), T_{z}(u)(x)=u(x-z)$ para $u \in S^{A}\left(\mathbb{R}^{n}\right)$ (ver capítulo 1 e notação (2.1)). Além disso,

$$
E_{z, \zeta}^{-1} u(x)=T_{-z} M_{-\zeta} u(x)=e^{-i \zeta(x+z)} u(x+z) .
$$

Dada $g \in S^{A}\left(\mathbb{R}^{n}\right)$

$$
\begin{gathered}
\widehat{E_{z, \zeta}(g)}(\xi)=\int e^{-i \xi y} E_{z, \zeta} g(y) d y= \\
=\int e^{-i \xi y} e^{i \zeta y} g(y-z) d y=\int e^{-i(\xi-\zeta)(y+z)} g(y) d y=e^{-i z(\xi-\zeta)} \hat{g}(\xi-\zeta) .
\end{gathered}
$$

Portanto, por $[\mathbf{1},(3.1)]$

$$
\begin{gathered}
\left(L_{h}\right)_{z, \zeta} g(x)=e^{-i \zeta(x+z)} L_{h} E_{z, \zeta} g(x+z)=e^{-i \zeta(x+z)} \int e^{i(x+z) \xi} h(x+z-J \xi) \widehat{E_{z, \zeta} g}(\xi)= \\
=e^{-i \zeta(x+z)} \int e^{i x \xi} e^{i \zeta z} h(x+z-J \xi) \hat{g}(\xi-\zeta) d \xi=e^{-i \zeta x} \int e^{i x(\xi+\zeta)} h(x+z-J(\xi+\zeta)) \hat{g}(\xi) d \xi= \\
=\int e^{i x \xi} h(x+z-J \zeta-J \xi) \hat{g}(\xi) d \xi .
\end{gathered}
$$

Isto é,

$$
\left(L_{h}\right)_{z, \zeta} g(x)=\int e^{i x \xi} h(x+z-J \zeta-J \xi) \hat{g}(\xi) d \xi
$$

para qualquer $(z, \zeta) \in \mathbb{R}^{2 n}$.

Logo,

$$
\left(L_{h}\right)_{z-J \zeta, 0} g(x)=\int e^{i x \xi} h(x+(z-J \zeta)-J \xi) \hat{g}(\xi) d \xi .
$$

Portanto, para qualquer $g \in S^{A}\left(\mathbb{R}^{n}\right),\left(L_{h}\right)_{z, \zeta}(g)=\left(L_{h}\right)_{z-J \zeta, 0}(g)$. Como $L_{h}$ é contínua em $\overline{S^{A}\left(\mathbb{R}^{n}\right)}$, temos $\left(L_{h}\right)_{z, \zeta}=\left(L_{h}\right)_{z-J \zeta, 0}$ 
Corolário 2.11. Dada $F \in \overline{S^{A}\left(\mathbb{R}^{n}\right)}$, temos $\left(L_{F}\right)_{z, \zeta}=\left(L_{F}\right)_{z-J \zeta, 0}$, como operadores de $S^{A}\left(\mathbb{R}^{n}\right)$ em $\overline{S^{A}\left(\mathbb{R}^{n}\right)}$.

Demonstração. Sejam $U=E_{z, \zeta}$ e $V=E_{z-J \zeta, 0}$. Notemos que, dada $h \in S^{A}\left(\mathbb{R}^{n}\right)$, $U(h)$ e $V(h)$ pertencem a $S^{A}\left(\mathbb{R}^{n}\right)$. Logo, por (2.9) e (2.10), temos

$$
\left(L_{F}\right)_{z, \zeta}=\left(L_{F}\right)_{z-J \zeta, 0}
$$

Teorema 2.12. Seja $T \in \mathcal{B}^{*}\left(\overline{S^{A}\left(\mathbb{R}^{n}\right)}\right)$ com $T R_{g}=R_{g} T$ para toda $g \in S^{A}\left(\mathbb{R}^{n}\right)$. Se $T$ é suave por translações, então $T$ é Heisenberg-suave.

Demonstração. Pelo corolário (2.7), temos que existe uma seqüência $\left(F_{k}\right)$ em $\overline{S^{A}\left(\mathbb{R}^{n}\right)}$ tal que $\lim _{k \rightarrow \infty} L_{F_{k}}(g)=T(g), \quad \forall g \in S^{A}\left(\mathbb{R}^{n}\right)$. Isto implica que para $(z, \zeta) \in \mathbb{R}^{2 n}$ temos $\lim _{k \rightarrow \infty}\left(L_{F_{k}}\right)_{z, \zeta}(g)=T_{z, \zeta}(g), \quad \forall g \in S^{A}\left(\mathbb{R}^{n}\right)$. Além disso, pelo (2.11), temos que $\lim _{k \rightarrow \infty}\left(L_{F_{k}}\right)_{z, \zeta}(g)=\lim _{k \rightarrow \infty}\left(L_{F_{k}}\right)_{z-J \zeta, 0}(g), \forall g \in S^{A}\left(\mathbb{R}^{n}\right) . \operatorname{Assim}, T_{z, \zeta}(g)=T_{z-J \zeta, 0}(g)$, $\forall g \in S^{A}\left(\mathbb{R}^{n}\right)$ e, como $T$ é contínua, temos $T_{z, \zeta}=T_{z-J \zeta, 0}$.

Como $T$ é suave por translações, a aplicação

$$
(z, \zeta) \longrightarrow z-J \zeta \longrightarrow T_{z-J \zeta, 0}
$$

é $C^{\infty}$, ou seja $(z, \zeta) \longrightarrow T_{z, \zeta}$ é $C^{\infty}$ 


\section{Capítulo 3}

Neste capítulo veremos uma generalização do teorema de Calderón-Vaillancourt [10] para operadores pseudo-diferenciais com símbolos tomando valores numa $C^{*}$-álgebra separável, baseada na demonstração feita por Seiler em [11].

Dadas uma $C^{*}$-álgebra separável $A$ e uma função a em $C B^{\infty}\left(\mathbb{R}^{2 n}, A\right)$, veremos que o operador $a(x, D)$ dado por

$$
a(x, D) u(x)=\int e^{i(x-y) \xi} a(x, \xi) u(y) d y \not \xi \text { para } u \operatorname{em} S^{A}\left(\mathbb{R}^{n}\right)
$$

é contínuo com a norma \|\|$_{2} ;\|u\|_{2}=\left\|\int u(x)^{*} u(x) d x\right\|^{\frac{1}{2}}$.

A integral em (I) deve ser entendida como uma integral oscilatória. Mais precisamente, para cada $x \in \mathbb{R}^{n}, a(x, D) u(x)=\lim _{k, m \rightarrow \infty} \iint e^{i(x-y) \xi} a(x, \xi) u(y) \psi_{m}(y) \psi_{k}^{\prime}(\xi) d y d \xi$, onde $\psi_{m}, \psi_{k}^{\prime}$ são as seqüências introduzidas na segunda página do capítulo 1. Demonstrase, então, que a integral oscilatória é igual à integral iterada (não necessariamente absolutamente convergente) $\int e^{i x \xi} a(x, \xi)\left[\int e^{-i y \xi} u(y) d y\right] d \xi$.

Observação 3.1. Notemos que o operador dado por

$$
a(x, D)_{R} u(x)=\int e^{i(x-y) \xi} u(y) a(x, \xi) d y d \xi, \quad u \in S^{A}\left(\mathbb{R}^{n}\right),
$$

não é um homomorfismo de módulos à direita em $\overline{S^{A}\left(\mathbb{R}^{n}\right)}$, se $A$ não é comutativa, pois, $\operatorname{dados} b \in A, u \in S^{A}\left(\mathbb{R}^{n}\right), a(x, D)_{R}(u b)(x)=\int e^{i(x-y) \xi} u(y) b a(x, \xi) d y d \xi$ e $a(x, D)_{R}(u)$ $b(x)=\int e^{i(x-y) \xi} u(y) a(x, \xi) b \not d y d \xi$. Ou seja, em geral, $a(x, D)_{R}(u b)$ e $a(x, D)_{R}(u) b$ não são iguais. 
Definição 3.2. Definimos a transformada de Fourier de $u$ em $S^{A}\left(\mathbb{R}^{n}\right)$ como segue:

$$
\hat{u}(\xi)=\int e^{-i y \xi} u(y) d y .
$$

Observação 3.3. Temos (como no caso escalar) que a transformada de Fourier é um operador contínuo e inversível em $S^{A}\left(\mathbb{R}^{n}\right)$. Além disso, é uma isometria com relação à norma \|\|$_{2}$, (corolário B.4). Assim, $a(x, D) u(x)=\int e^{i x \xi} a(x, \xi) \hat{u}(\xi) d \xi$.

Observação 3.4. Notemos que, pelo Teorema da Convergência Dominada (A.7), $a(x, D)$ $u$ é uma função contínua, pois $\left\|e^{i x \xi} a(x, \xi) \hat{u}(\xi)\right\| \leq\|a\|_{\infty}\|\hat{u}(\xi)\| \in L^{1}\left(\mathbb{R}^{n}\right)$.

Definição 3.5. Dada $a \in C B^{\infty}\left(\mathbb{R}^{2 n}, A\right)$, seja $\pi(a)=\sup \left\{\left\|\partial_{y}^{\beta} \partial_{\eta}^{\gamma} a\right\|_{\infty}, \beta, \gamma \leq(1,1, \cdots, 1)\right\}$.

Notação 3.6. $\alpha=(1,1, \cdots, 1) \in \mathbb{N}^{n}$.

Lema 3.7. Seja $\phi \in C_{c}^{\infty}\left(\mathbb{R}^{2 n}\right)$ com $\phi \equiv 1$ perto do zero. Dada $a \in C B^{\infty}\left(\mathbb{R}^{2 n}, A\right)$, seja $a_{\varepsilon}(y, \eta)=\phi(\varepsilon y, \varepsilon \eta) a(y, \eta), 0<\varepsilon \leq 1$. Então existe uma constante $c_{1} \in \mathbb{R}^{+}$, independente de $a$ e $\varepsilon$, tal que $\pi\left(a_{\varepsilon}\right) \leq c_{1} \pi(a)$.

Demonstração. Dados os multi-índices $\beta, \gamma \leq \alpha$,

$$
\begin{aligned}
\partial_{y}^{\beta} \partial_{\eta}^{\gamma} a_{\varepsilon}(y, \eta)= & \partial_{y}^{\beta}\left[\partial_{\eta}^{\gamma} \phi(\varepsilon y, \varepsilon \eta) a(y, \eta)\right]=\partial_{y}^{\beta}\left[\sum_{\gamma_{1}+\gamma_{2}=\gamma} \partial_{\eta}^{\gamma_{1}} \phi(\varepsilon y, \varepsilon \eta) \partial_{\eta}^{\gamma_{2}} a(y, \eta)\right]= \\
& =\sum_{\beta_{1}+\beta_{2}=\beta} \sum_{\gamma_{1}+\gamma_{2}=\gamma} \partial_{y}^{\beta_{1}} \partial_{\eta}^{\gamma_{1}} \phi(\varepsilon y, \varepsilon \eta) \partial_{y}^{\beta_{2}} \partial_{\eta}^{\gamma_{2}} a(y, \eta) .
\end{aligned}
$$

Portanto, se $\phi_{\varepsilon}(x, y)=\phi(\varepsilon y, \varepsilon x)$, temos:

$$
\left\|\partial_{y}^{\beta} \partial_{\eta}^{\gamma} a_{\varepsilon}\right\|_{\infty} \leq \sum_{\beta_{1} \leq \beta} \sum_{\gamma_{1} \leq \gamma}\left\|\partial_{y}^{\beta_{1}} \partial_{\eta}^{\gamma_{1}} \phi_{\varepsilon}\right\|_{\infty} \pi(a) .
$$

Assim,

$$
\pi\left(a_{\varepsilon}\right) \leq \sum_{\beta, \gamma \leq \alpha} \varepsilon^{|\beta+\gamma|}\left\|\partial_{y}^{\beta} \partial_{\eta}^{\gamma} \phi\right\|_{\infty} \pi(a) \leq \sum_{\beta, \gamma \leq \alpha}\left\|\partial_{y}^{\beta} \partial_{\eta}^{\gamma} \phi\right\|_{\infty} \pi(a)
$$

Nota 3.8. Dadas $f, g \in L^{2}\left(\mathbb{R}^{n}, A\right)$ (ver A.9), $(f \mid g)=\int f(x)^{*} g(x) d x$ está bem definido, pois $f^{*} g \in L^{1}\left(\mathbb{R}^{n}, A\right)$ (ver A.9) por Hölder $[\mathbf{3}, 6.2]$. E temos que $(\cdot \mid \cdot): L^{2}\left(\mathbb{R}^{n}, A\right) \times$ $L^{2}\left(\mathbb{R}^{n}, A\right) \rightarrow A$ é contínua como função das duas variáveis, pois $\|(f \mid g)\| \leq\|f\|_{L^{2}}\|g\|_{L^{2}}$. Notemos que, se $h_{1}, h_{2} \in S^{A}\left(\mathbb{R}^{n}\right),\left(h_{1} \mid h_{2}\right)=<h_{1}, h_{2}>$. E temos que $\|h\|_{2} \leq\|h\|_{L_{2}}$, para $h \in S^{A}\left(\mathbb{R}^{n}\right)$. 
Proposição 3.9. $L^{2}\left(\mathbb{R}^{n}, A\right) \subseteq \overline{S^{A}\left(\mathbb{R}^{n}\right)}$ e a inclusão é contínua.

Demonstração. Definamos $\iota:\left(S^{A}\left(\mathbb{R}^{n}\right),\|\cdot\|_{L_{2}}\right) \rightarrow \overline{S^{A}\left(\mathbb{R}^{n}\right)}$ por $\iota(h)=h, h \in S^{A}\left(\mathbb{R}^{n}\right)$. Notemos que $\|\iota(h)\|_{2}=\|h\|_{2} \leq\|h\|_{L_{2}}$. Portanto, $\iota$ é contínua.

Seja $\mathcal{I}: L^{2}\left(\mathbb{R}^{n}, A\right) \rightarrow \overline{S^{A}\left(\mathbb{R}^{n}\right)}$ a única extensão contínua de $\iota$. Provemos que $\mathcal{I}$ é injetora; ou seja que se $\mathcal{I}(f)=0 \Rightarrow f=0$, para $f \in L^{2}\left(\mathbb{R}^{n}, A\right)$.

Dada $f \in L^{2}\left(\mathbb{R}^{n}, A\right)$, seja $\left(f_{m}\right)$ uma seqüência em $S^{A}\left(\mathbb{R}^{n}\right)$ tal que $\lim _{m \rightarrow \infty} \| f_{m}-$ $f \|_{L_{2}}=0$ (ver A.19). Para $g \in L^{2}\left(\mathbb{R}^{n}, A\right)$ temos que (por Hölder): $\left\|\left(f_{m}-f \mid g\right)\right\| \leq$ $\int\left\|\left(f_{m}-f\right)(x)^{*} g(x)\right\| d x \leq\left\|f_{m}-f\right\|_{L_{2}}\|g\|_{L_{2}}$. Logo,

$$
\left(f_{m} \mid g\right) \rightarrow(f \mid g), \quad \forall g \in L^{2}\left(\mathbb{R}^{n}, A\right) .
$$

Se $\mathcal{I}(f)=0$, como $\mathcal{I}$ è contìnua, $\mathcal{I}\left(f_{m}\right) \rightarrow 0$, ou seja, $\left\|f_{m}\right\|_{2} \rightarrow 0$. Assim, dada $h \in S^{A}\left(\mathbb{R}^{n}\right),\left\|<f_{m}, h>\right\| \leq\left\|f_{m}\right\|_{2}\|h\|_{2} \rightarrow 0$ por Cauchy-Schwartz [6, lemma 1.1.2]. Ou seja,

$$
\mathcal{I}(f)=0 \Rightarrow<f_{m}, h>\rightarrow 0, \quad \forall h \in S^{A}\left(\mathbb{R}^{n}\right)
$$

Suponhamos $\mathcal{I}(f)=0$. Então, dada $h \in S^{A}\left(\mathbb{R}^{n}\right)$, por (1) e (2) temos $(f \mid h)=0$; em particular, para todo $m \in \mathbb{N},\left(f \mid f_{m}\right)=0$. Novamente por (1), tomando $g=f$, temos que $\left(f \mid f_{m}\right) \rightarrow(f \mid f)$ e, portanto, $(f \mid f)=0$, ou seja, $f=0$, (A.17) e $\mathcal{I}$ é injetora.

Corolário 3.10. Se $f, g \in L^{2}\left(\mathbb{R}^{n}, A\right)$, então $<f, g>=(f \mid g)$. Em particular, $\|f\|_{2}=$ $\left\|\int f(x)^{*} f(x) d x\right\|^{\frac{1}{2}}$.

Demonstração. Sejam $\left(f_{m}\right)$ e $\left(g_{k}\right)$ seqüências em $S^{A}\left(\mathbb{R}^{n}\right)$ que convergem em $L^{2}\left(\mathbb{R}^{n}, A\right)$ para $f$ e $g$, respectivamente. Como a inclusão $L^{2}\left(\mathbb{R}^{n}, A\right) \subseteq \overline{S^{A}\left(\mathbb{R}^{n}\right)}$ é contínua, (pela proposição 3.9$),\left(f_{m}\right)$ e $\left(g_{k}\right)$ convergem em $\overline{S^{A}\left(\mathbb{R}^{n}\right)}$ para $f$ e $g$, respectivamente. Portanto, (ver 3.8),

$$
(f \mid g) \longleftarrow\left(f_{m} \mid g_{k}\right)=<f_{m}, g_{k}>\longrightarrow<f, g>.
$$

Isto é $<f, g>=\int f(x)^{*} g(x) d x$.

Observação 3.11. Se $D_{y_{j}}=-i \partial_{y_{j}}$, vale que $(i+x)^{\alpha} e^{i x y}=\left(i+D_{y}\right)^{\alpha} e^{i x y}, \quad x, y \in \mathbb{R}^{n}$. (Escrevemos $(i+x)$ querendo dizer $(i \alpha+x))$. 
Proposição 3.12. Dadas $a \in C B^{\infty}\left(\mathbb{R}^{2 n}, A\right)$ e $u \in S^{A}\left(\mathbb{R}^{n}\right)$, então $a(x, D)(u) \in$ $L^{2}\left(\mathbb{R}^{n}, A\right)$.

Demonstração. Notemos que, pela observação $3.4, a(x, D)(u)(x)$ é uma função contínua de $\mathbb{R}^{n}$ em $A$ e, portanto, mensurável.

$$
\begin{gathered}
a(x, D)(u)(x)=\int e^{i x \eta} a(x, \eta) \hat{u}(\eta) d \eta=\int(i+x)^{-\alpha}\left[(i+x)^{\alpha} e^{i x \eta}\right] a(x, \eta) \hat{u}(\eta) d \eta= \\
=(i+x)^{-\alpha} \int\left[\left(i+D_{\eta}\right)^{\alpha} e^{i x \eta}\right] a(x, \eta) \hat{u}(\eta) d \eta=(i+x)^{-\alpha} \int e^{i x \eta}\left[\left(i-D_{\eta}\right)^{\alpha} a(x, \eta) \hat{u}(\eta)\right] d \eta
\end{gathered}
$$

(para a última igualdade fizemos integração por partes, ver A.15).

Como a $\in C B^{\infty}\left(\mathbb{R}^{2 n}, A\right)$ e $\hat{u} \in S^{A}\left(\mathbb{R}^{n}\right)$, (3.3) , a última integral é limitada; digamos que, em norma, é menor ou igual que $c_{2} \in \mathbb{R}^{+}$. Veremos na observação (3.14) que $c_{2}$ depende de $a$ e de $u$, mas não depende de $x$. Assim, temos $\|a(x, D)(u)\|_{L_{2}}^{2} \leq c_{2}^{2} \int|i+x|^{-2|\alpha|} d x$ e portanto $a(x, D)(u) \in L^{2}\left(\mathbb{R}^{n}, A\right)$.

Corolário 3.13. Pela Proposição $(3.9) \quad a(x, D)(u) \in \overline{S^{A}\left(\mathbb{R}^{n}\right)}$.

Observação 3.14. Sobre a constante $c_{2}$ da proposição (3.12) .

$$
\left\|\int e^{i x \eta}\left[\left(i-D_{\eta}\right)^{\alpha} a(x, \eta) \hat{u}(\eta)\right] d \eta\right\| \leq \int\left\|\left(i-D_{\eta}\right)^{\alpha} a(x, \eta) \hat{u}(\eta)\right\| d \eta
$$

e temos que $\left(i-D_{\eta}\right)^{\alpha} a(x, \eta) \hat{u}(\eta)$ é soma de termos do tipo $D_{\eta}^{\alpha_{1}} a(x, \eta) D_{\eta}^{\alpha_{2}} \hat{u}(\eta)$ (multiplicados por uma potência de $i$ ) e vale

$$
\left\|D_{\eta}^{\alpha_{1}} a(x, \eta) D_{\eta}^{\alpha_{2}} \hat{u}(\eta)\right\| \leq \pi(a)\left\|D_{\eta}^{\alpha_{2}} \hat{u}(\eta)\right\|,
$$

onde $\alpha_{1}, \alpha_{2} \leq \alpha$. Assim, $\left\|\int e^{i x \eta}\left[\left(i-D_{\eta}\right)^{\alpha} a(x, \eta) \hat{u}(\eta)\right] d \eta\right\| \leq M \pi(a)$, onde $M \in \mathbb{R}^{+}$é uma constante (que depende de $n$ e de $u$ ) que tem a ver com a soma citada acima (número de termos e módulo das constantes) e com a soma das integrais do tipo $\int\left\|D_{\eta}^{\alpha_{2}} \hat{u}(\eta)\right\| d \eta$. Logo, a constante $c_{2}$ mencionada acima é $M \pi(a)$, onde $M$ não depende de $a$ nem de $x$.

A seguinte proposição foi baseada no lema 3.13 de [11]. 
Proposição 3.15. Dados $a$ e $a_{\varepsilon}$ como no lema (3.7) e $u, v \in S^{A}\left(\mathbb{R}^{n}\right)$, vale que

$$
<v, a_{\varepsilon}(x, D) u>\underset{\varepsilon \rightarrow 0}{\longrightarrow}<v, a(x, D) u>\text { em } A
$$

Demonstração. Sejam $b_{\varepsilon}=a_{\varepsilon}-a \quad\left(b_{\varepsilon} \in C B^{\infty}\left(\mathbb{R}^{2 n}, A\right)\right)$ e $B_{\varepsilon}=b_{\varepsilon}(x, D)$. Provemos que $\left\langle v, B_{\varepsilon} u>\underset{\varepsilon \rightarrow 0}{\longrightarrow} 0 B_{\varepsilon} u(x)=\int e^{i x \eta} b_{\varepsilon}(x, \eta) \hat{u}(\eta) d \eta\right.$, e temos que $\left\|e^{i x \eta} b_{\varepsilon}(x, \eta) \hat{u}(\eta)\right\| \leq$ $\pi\left(b_{\varepsilon}\right)\|\hat{u}(\eta)\|$.

Observemos que, pelo lema (3.7), existe $c_{1} \in \mathbb{R}^{+}$, independente de $b_{\varepsilon}$, tal que $\pi\left(b_{\varepsilon}\right)=\pi\left(a_{\varepsilon}-a\right) \leq \pi\left(a_{\varepsilon}\right)+\pi(a) \leq\left(c_{1}+1\right) \pi(a)$. Portanto, $\left\|e^{i x \eta} b_{\varepsilon}(x, \eta) \hat{u}(\eta)\right\| \leq\left(c_{1}+\right.$ 1) $\pi(a)\|\hat{u}(\eta)\|$.

Como $\left\|e^{i x \eta} b_{\varepsilon}(x, \eta) \hat{u}(\eta)\right\| \rightarrow 0$ e $\int\|\hat{u}(\eta)\| d \eta<\infty$, pelo Teorema da Convergência Dominada, $\left\|B_{\varepsilon} u(x)\right\| \rightarrow 0$, para cada $x \in \mathbb{R}^{n}$.

Por outro lado, como visto na proposição (3.12) e na observação (3.14), existe uma constante $M \in \mathbb{R}^{+}$, independente de $\varepsilon$, tal que

$$
\int\left\|B_{\varepsilon} u(x)\right\|^{2} d x \leq M^{2} \pi\left(b_{\varepsilon}\right)^{2} \int|i+x|^{-2|\alpha|} d x \leq M^{2}\left(c_{1}+1\right)^{2} \pi(a)^{2} \int|i+x|^{-2|\alpha|} d x<\infty .
$$

Novamente, pelo Teorema da Convergência Dominada, $\left\|B_{\varepsilon} u\right\|_{L_{2}} \rightarrow 0$. Isto implica que $\left\|B_{\varepsilon} u\right\|_{2} \rightarrow 0$ por (3.8). Finalmente, por Cauchy-Schwarz, como $\left.\|<v, B_{\varepsilon} u\right\rangle \| \leq$ $\|v\|_{2}\left\|B_{\varepsilon} u\right\|_{2}$, temos que $<v, a_{\varepsilon}(x, D) u>\rightarrow<v, a(x, D) u>$ em $A$.

Nota 3.16. Dada $a \in C B^{\infty}\left(\mathbb{R}^{2 n}, A\right)$, pelo visto na proposição $(3.12), a(x, D)(u) \in$ $L^{2}\left(\mathbb{R}^{n}, A\right)$, para $u \in S^{A}\left(\mathbb{R}^{n}\right)$. Assim, pela nota $(3.8),<v, a(x, D) u>$ está bem definido para $v \in S^{A}\left(\mathbb{R}^{n}\right)$.

Lema 3.17. Dada $u \in S^{A}\left(\mathbb{R}^{n}\right)$ seja $f(x, \xi)=\int e^{-i y \xi}(i+x-y)^{-\alpha} u(y) d y$. Então, $f \in L^{2}\left(\mathbb{R}^{2 n}, A\right)$.

Demonstração. Como para qualquer $N \in \mathbb{N}$

$$
\frac{\left(1-\Delta_{y}\right)^{N} e^{-i y \xi}}{\left(1+|\xi|^{2}\right)^{N}}=e^{-i y \xi}
$$

temos

$$
f(x, \xi)=\frac{1}{\left(1+|\xi|^{2}\right)^{N}} \int\left[\left(1-\Delta_{y}\right)^{N} e^{-i y \xi}\right](i+x-y)^{-\alpha} u(y) d y
$$


Queremos provar que a integral acima é combinação linear de termos do tipo

$$
\int e^{-i y \xi} \partial_{y}^{\beta} u(y)(i+x-y)^{-\gamma} d y, \quad \beta \geq 0 e \gamma \geq \alpha
$$

Para isto, calculemos, para $j=1, \cdots, n, M, P \leq N$ :

$$
\int \partial_{y_{j}}\left[\left(1-\Delta_{y}\right)^{M} e^{-i y \xi}\right]\left[\left(1-\Delta_{y}\right)^{P} u(y)(i+x-y)^{-\alpha}\right] d y=(*) .
$$

Para $(x, \xi)$ fixo, o integrando acima é uma função de $L^{1}\left(\mathbb{R}^{n}, A\right)$, pois $u \in S^{A}\left(\mathbb{R}^{n}\right)$. Sejam $g(y)=\left(1-\Delta_{y}\right)^{M} e^{-i y \xi}$ e $h(y)=\left(1-\Delta_{y}\right)^{P} u(y)(i+x-y)^{-\alpha}$. Pelo corolário A.16, temos que

$$
\int\left[\partial_{y_{1}} g(y)\right](y) d y=-\int g(y)\left(\partial_{y_{1}} h(y)\right) d y .
$$

Pelo Teorema de Fubini (A.13), temos, tomando $j=1$ sem perda de generalidade:

$$
\begin{aligned}
& (*)=\int_{\mathbb{R}^{n-1}}\left(\int_{-\infty}^{+\infty} \partial_{y_{1}} g(y) h(y) d y_{1}\right) d y_{2} \cdots d y_{n}= \\
& =\int_{\mathbb{R}^{n-1}}\left[\lim _{R \rightarrow+\infty} g\left(R, y_{2}, \cdots, y_{n}\right) h\left(R, y_{2}, \cdots, y_{n}\right)-\right. \\
& \left.\lim _{R \rightarrow-\infty} g\left(R, y_{2}, \cdots, y_{n}\right) h\left(R, y_{2}, \cdots, y_{n}\right)\right] d y_{2}, \cdots, d y_{n}
\end{aligned}
$$

pelo Teorema Fundamental do Cálculo, A.14.

Cada um dos limites acima é igual a zero. Portanto,

$$
\int\left[\partial_{y_{1}} g(y)\right] h(y) d y=-\int g(y)\left[\partial_{y_{1}} h(y)\right] d y,
$$

isto é, podemos fazer a integração por partes. Deste modo, temos que $f(x, \xi)$ é combinação linear de termos do tipo

$$
\frac{1}{\left(1+|\xi|^{2}\right)^{N}} \int e^{-i y \xi} \partial_{y}^{\beta} u(y)(i+x-y)^{-\gamma} d y, \quad \beta \geq 0, \gamma \geq \alpha,
$$

onde a integral é finita pois $u \in S^{A}\left(\mathbb{R}^{n}\right)$.

Por outro lado, pela desigualdade de Petree $([\mathbf{1 5},(3.6)])$, temos que

$$
\left|(i+x-y)^{-\gamma}\right| \leq 4^{n} \frac{\sqrt{1+y_{1}^{2}} \cdots \sqrt{1+y_{n}^{2}}}{\sqrt{1+x_{1}^{2}} \cdots \sqrt{1+x_{n}^{2}}} .
$$


E, além disso,

$\partial_{y_{1}}(i+x-y)^{-\gamma}=-\gamma_{1}\left(i+x_{1}-y_{1}\right)^{-\gamma_{1}-1}\left(i+x_{2}-y_{2}\right)^{-\gamma_{2}} \cdots\left(i+x_{n}-y_{n}\right)^{-\gamma_{n}}$.

Deste modo, $\|f(x, \xi)\|$ é menor ou igual que uma combinação linear de termos do tipo

$$
\frac{4^{n}}{\left(1+|\xi|^{2}\right)^{N} \sqrt{1+x_{1}^{2}} \cdots \sqrt{1+x_{n}^{2}}} \int\left\|\partial_{y}^{\beta} u(y)\right\| \sqrt{1+y_{1}^{2}} \cdots \sqrt{1+y_{n}^{2}} d y, \quad \beta \geq 0
$$

que pertence a $L^{2}\left(\mathbb{R}^{2 n}\right)$ para $N \geq \frac{n}{2}$. Então $f \in L^{2}\left(\mathbb{R}^{2 n}, A\right)$.

Lema 3.18. Dada $u \in S^{A}\left(\mathbb{R}^{n}\right)$, seja $f(x, \xi)=\int e^{-i y \xi}(i+x-y)^{-\alpha} u(y) d y$. Então existe $c_{3} \in \mathbb{R}^{+}$, independente de $f$ e de $u$, tal que $\|f\|_{2} \leq c_{3}\|u\|_{2}$.

Demonstração. Sejam $h(z)=(i-z)^{-\alpha}$ e $h_{z}(y)=h(y-z)$; assim, $f(x, \xi)=$ $\int e^{-i y \xi} h_{x}(y) u(y) d y=\widehat{h_{x} u}(\xi)$. (Notemos que $h_{x} u \in S^{A}\left(\mathbb{R}^{n}\right)$.) Como a transformada de Fourier é um operador "unitário" em $S^{A}\left(\mathbb{R}^{n}\right)$ (B.3), temos que, dados $g, p \in S^{A}\left(\mathbb{R}^{n}\right)$, $<\hat{p}, \hat{g}>=<p, g>$.

Vimos no lema (3.17) que $f \in L^{2}\left(\mathbb{R}^{2 n}, A\right)$, logo, pelo Teorema de Fubini, (A.13), temos

$$
\begin{gathered}
\int f(x, \xi)^{*} f(x, \xi) d x d \xi=\int\left[\widehat{h_{x} u}(\xi)\right]^{*} \widehat{h_{x} u}(\xi) d \xi d x=\int<\widehat{h_{x} u}, \widehat{h_{x} u}>d x= \\
=\int<h_{x} u, h_{x} u>d x=\int u(\xi)^{*} \overline{h_{x}(\xi)} h_{x}(\xi) u(\xi) d \xi d x=\int u(\xi)^{*} \int \overline{h_{x}(\xi)} h_{x}(\xi) d x u(\xi) d \xi= \\
=\int u(\xi)^{*} \int \overline{h(\xi-x)} h(\xi-x) d x u(\xi) d \xi=\int \overline{h(x)} h(x) d x \int u(\xi)^{*} u(\xi) d \xi .
\end{gathered}
$$

Logo, se $c_{3}=\|h\|_{L_{2}},(3.9)$, temos $\|f\|_{2} \leq c_{3}\|u\|_{2}$.

O próximo teorema foi enunciado e demonstrado baseando-nos no teorema 3.14 de $[\mathbf{1 1}]$. 
Teorema 3.19. Dada $a \in C B^{\infty}\left(\mathbb{R}^{2 n}, A\right)$, então a induz um operador contínuo em $S^{A}\left(\mathbb{R}^{n}\right)$ como pré-módulo de Hilbert. Além disso, existe uma constante $k \in \mathbb{R}^{+}$, independente de $a$, tal que $\|a(x, D)\| \leq k \pi(a)$.

Demonstração. Suponhamos, primeiramente, que a tem suporte compacto. Seja $T=a(x, D)$. Calculemos, para $u, v \in S^{A}\left(\mathbb{R}^{n}\right),<\hat{v}, \widehat{T u}>=<v, T u>$.

$$
\begin{gathered}
T u(x)=\int e^{i \xi(x-y)} a(x, \xi) u(y) d y d \xi \\
\widehat{T u}(\eta)=\int e^{-i x \eta} e^{i \xi(x-y)} a(x, \xi) u(y) d y d \xi d x \\
<\hat{v}, \widehat{T u}>=\int e^{-i x \eta} e^{i \xi(x-y)} \hat{v}(\eta)^{*} a(x, \xi) u(y) d y d \xi d x d \eta= \\
=\int e^{-i x \eta}(i+x-y)^{-\alpha} \hat{v}(\eta)^{*}\left[(i+x-y)^{\alpha} e^{i \xi(x-y)}\right] a(x, \xi) u(y) d \xi d x d y d \eta .
\end{gathered}
$$

Como a tem suporte compacto e $u, v \in S^{A}\left(\mathbb{R}^{n}\right)$, vale Fubini (A.13), o que justifica as igualdades acima; além disso, podemos aplicar a integração por partes e, pela observação (3.11) , temos:

$$
\begin{gathered}
<\hat{v}, \widehat{T u}>=\int e^{-i x \eta}(i+x-y)^{-\alpha} \hat{v}(\eta)^{*}\left[\left(i+D_{\xi}\right)^{\alpha} e^{i \xi(x-y)}\right] a(x, \xi) u(y) d \xi d x d y d \eta= \\
=\int e^{-i x \eta}(i+x-y)^{-\alpha} v(\eta)^{*} e^{i \xi x} e^{-i \xi y}\left[\left(i-D_{\xi}\right)^{\alpha} a(x, \xi)\right] u(y) d \xi d x d y d \eta= \\
=\int e^{-i y \xi}(i+x-y)^{-\alpha}(i+\xi-\eta)^{-\alpha}\left[(i+\xi-\eta)^{\alpha} e^{i x(\xi-\eta)}\right] \hat{v}(\eta)^{*}\left[\left(i-D_{\xi}\right)^{\alpha} a(x, \xi)\right] u(y) d \xi d x d y d \eta= \\
=\int e^{-i y \xi}(i+x-y)^{-\alpha}(i+\xi-\eta)^{-\alpha}\left[\left(i+D_{x}\right)^{\alpha} e^{i x(\xi-\eta)}\right] \hat{v}(\eta)^{*}\left[\left(i-D_{\xi}\right)^{\alpha} a(x, \xi)\right] u(y) d x d \xi d y d \eta=
\end{gathered}
$$




$$
\begin{gathered}
=\int e^{-i y \xi}(i+x-y)^{-\alpha} e^{-i x \eta}(i+\xi-\eta)^{-\alpha} e^{i x \xi} \hat{v}(\eta)^{*}\left[\left(i-D_{x}\right)^{\alpha}\left(i-D_{\xi}\right)^{\alpha} a(x, \xi)\right] u(y) d x d \xi d y d \eta= \\
=\int e^{i x \xi}\left(e^{-i x \eta} h_{\xi}(\eta) \hat{v}(\eta)^{*}\left[\left(i-D_{x}\right)^{\alpha}\left(i-D_{\xi}\right)^{\alpha} a(x, \xi)\right]\left(e^{-i y \xi} h_{x}(y) u(y)\right) d y d \eta d x d \xi\right.
\end{gathered}
$$

usando a notação do lema (3.18) .

Sejam

$$
\begin{aligned}
& f(x, \xi)=\int e^{-i x \eta} h_{\xi}(\eta) \hat{v}(\eta)^{*} d \eta \\
& g(x, \xi)=\int e^{-i y \xi} h_{x}(y) u(y) d y
\end{aligned}
$$

Então:

$$
<\hat{v}, \widehat{T u}>=\int e^{i x \xi} f(x, \xi)\left[\left(i-D_{x}\right)^{\alpha}\left(i-D_{\xi}\right)^{\alpha} a(x, \xi)\right] g(x, \xi) d x d \xi
$$

Abusando da notação, colocando as variáveis das funções, temos:

$$
\|<\hat{v}, \widehat{T u}>\|=\frac{1}{(2 \pi)^{\frac{n}{2}}}\left\|<e^{-i x \xi} f^{*}(x, \xi),\left[\left(i-D_{x}\right)^{\alpha}\left(i-D_{\xi}\right)^{\alpha} a(x, \xi)\right] g(x, \xi)>\right\| .
$$

Seja $c(x, \xi)=\left(i-D_{x}\right)^{\alpha}\left(i-D_{\xi}\right)^{\alpha} a(x, \xi)$. Como $a \in C B^{\infty}\left(\mathbb{R}^{2 n}, A\right)$, temos que

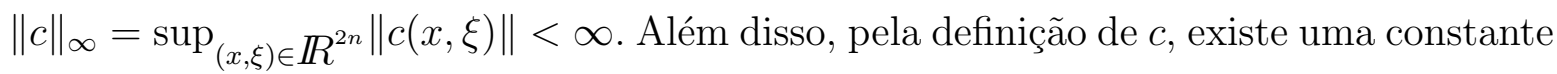
$l \in \mathbb{R}^{+}$, que não depende de $a$, tal que $\|c\|_{\infty} \leq l \pi(a)$. E temos, pela demonstração do teorema 2.2 .5 de $[5]$,

$$
\begin{gathered}
\int g(x, \xi)^{*} c(x, \xi)^{*} c(x, \xi) g(x, \xi) d x d \xi \leq \int\|c(x, \xi)\|^{2} g(x, \xi)^{*} g(x, \xi) d x d \xi \leq \\
\leq\|c\|_{\infty}^{2} \int g(x, \xi)^{*} g(x, \xi) d x d \xi
\end{gathered}
$$

Portanto, pelo lema (3.18), $\|c g\|_{2} \leq l \pi(a) c_{3}\|u\|_{2}$. 
Como $f^{*}(x, \xi)=\int e^{i x \eta} \overline{h_{\xi}(\eta)} \hat{v}(\eta) d \eta$, de um modo análogo ao feito na demonstração do lema (3.18), temos $\left\|f^{*}\right\|_{2} \leq c_{3}\|\hat{v}\|_{2}=c_{3}\|v\|_{2}$ (ver observação (3.3)). Logo, por Cauchy-Schwarz,

$$
\|<\hat{v}, \widehat{T u}>\| \leq \frac{l c_{3}^{2}}{(2 \pi)^{\frac{n}{2}}} \pi(a)\|u\|_{2}\|v\|_{2} .
$$

Tomando $k_{1}=\frac{l c_{3}^{2}}{(2 \pi)^{\frac{n}{2}}}$, temos que $\|<v, a(x, D) u>\| \leq k_{1} \pi(a)\|u\|_{2}\|v\|_{2}$, onde $k_{1}$ não depende de $a$, e então $a(x, D)$ é contínuo em $S^{A}\left(\mathbb{R}^{n}\right)$.

Para o caso geral, consideremos $a_{\varepsilon}$ como na proposição (3.15) . Então, pelo feito acima, temos:

$$
\|<v, a(x, D) u>\| \underset{\varepsilon \rightarrow 0}{\longleftarrow}\left\|<v, a_{\varepsilon}(x, D) u>\right\| \leq k_{1} \pi\left(a_{\varepsilon}\right)\|u\|_{2}\|v\|_{2}
$$

Portanto, pelo lema (3.7), $\|<v, a(x, D) u>\| \leq k_{1} c_{1} \pi(a)\|u\|_{2}\|v\|_{2}$. E, assim, existe $k \in \mathbb{R}^{+}, k=k_{1} c_{1}$, independente de $a$, tal que $\|<v, a(x, D) u>\| \leq k \pi(a)\|u\|_{2}\|v\|_{2}$.

Tomemos $v=a(x, D)(u)$. Como $v \in L^{2}\left(\mathbb{R}^{n}, A\right)$, proposição (3.12), pela nota (3.8) , a seguinte desigualdade faz sentido:

$$
\begin{aligned}
& \qquad<<a(x, D)(u), a(x, D)(u)>\|\leq k \pi(a)\| u\left\|_{2}\right\| a(x, D)(u) \|_{2}, \forall u \in S^{A}\left(\mathbb{R}^{n}\right) . \\
& \text { Portanto, }\|a(x, D)(u)\|_{2} \leq k \pi(a)\|u\|_{2} \text {, e temos }\|a(x, D)\| \leq k \pi(a) .
\end{aligned}
$$

Corolário 3.20. Nas condições do teorema, vemos que $a(x, D)$ se estende ao módulo de Hilbert $\overline{S^{A}\left(\mathbb{R}^{n}\right)}$, continuamente. 


\section{Capítulo 4}

No capítulo 1, vimos a demonstração da conjectura proposta por Rieffel no final do capítulo 4 de $[\mathbf{1}]$ para o caso da $C^{*}$-álgebra dos números complexos. Foi necessária a caracterização feita por Cordes ([2, capítulo 8]) dos operadores suaves pela ação do grupo de Heisenberg, os operadores pseudo-diferenciais. Neste capítulo, veremos que uma generalização da caracterização de Cordes para uma $C^{*}$ - álgebra, $A$, qualquer, implicaria que a conjectura proposta por Rieffel é verdadeira.

Suponhamos então que vale a seguinte generalização da caracterização de Cordes:

4.1. Seja $\mathcal{H} \mathcal{S}$ o conjunto dos operadores Heisenberg-suaves em $\mathcal{B}^{*}\left(\overline{S^{A}\left(\mathbb{R}^{n}\right)}\right)$. Então a aplicação $O: C B^{\infty}\left(\mathbb{R}^{2 n}, A\right) \rightarrow \mathcal{H S}$, dada por $O(a)=a(x, D)$ é uma bijeção, onde $a(x, D)$ é o operador definido em (I) do capítulo 3.

Vejamos que $O$ (acima) está bem definida.

Lema 4.2. Dado $a \in C B^{\infty}\left(\mathbb{R}^{2 n}, A\right)$, de suporte compacto, o operador $O(a)$ possui adjunto; ou seja, existe um operador $T$ em $\overline{S^{A}\left(\mathbb{R}^{n}\right)}$, contínuo, tal que

$$
<O(a) u, v>=<u, T v>\text {, se } u, v \in S^{A}\left(\mathbb{R}^{n}\right) .
$$

\section{Demonstração.}

$$
\begin{gathered}
<O(a) u, v>=\int\left[\int e^{i(x-y) \xi} a(x, \xi) u(y) d y d \xi\right]^{*} v(x) d x= \\
=\iint e^{i(y-x) \xi} u(y)^{*} a(x, \xi)^{*} d y d \xi v(x) d x=\int u(y)^{*}\left[\int e^{i(y-x) \xi} a(x, \xi)^{*} v(x) d x d \xi\right] d y,
\end{gathered}
$$


para a última igualdade aplicamos o Teorema de Fubini, A.13, pois a tem suporte compacto e $u, v \in S^{A}\left(\mathbb{R}^{n}\right)$.

Como $a$ tem suporte compacto, $a \in S^{A}\left(\mathbb{R}^{2 n}\right)$. Seja $F_{2}$ a transformada de Fourier, na segunda variável, definido em $S^{A}\left(\mathbb{R}^{2 n}\right)$. Com esta notação, seja

$$
c(y, z)=\int e^{i z \xi} a(y-z, \xi)^{*} d \xi=F_{2}^{-1} a^{*}(y-z, z)
$$

e temos $c \in S^{A}\left(\mathbb{R}^{2 n}\right)$.

Seja $p(y, z)=F_{2} c(y, z), \quad\left(p \in S^{A}\left(\mathbb{R}^{2 n}\right)\right.$, ver início do Apêndice B), então

$$
c(y, z)=F_{2}^{-1} p(y, z)
$$

Por $(1), \operatorname{temos} c(y, y-x)=\int e^{i(y-x) \xi} a(x, \xi)^{*} d \xi \mathrm{e}, \operatorname{por}(2), c(y, y-x)=\int e^{i(y-x) \xi} p(y, \xi) d \xi$. Portanto, $\int e^{i(y-x) \xi} a(x, \xi)^{*} v(x) d \xi d x=\int e^{i(y-x) \xi} p(y, \xi) v(x) d \xi d x$.

Assim,

$$
\int u(y)^{*}\left[\int e^{i(y-x) \xi} a(x, \xi)^{*} v(x) d x d \xi\right] d y=\int u(y)^{*}\left[\int e^{i(y-x)} p(y, \xi) v(x) d x d \xi\right] d y
$$

ou seja, $<O(a) u, v>=<u, O(p) v>, \quad \forall u, v \in S^{A}\left(\mathbb{R}^{n}\right)$.

Observação 4.3. Sobre a função $p$ do lema 4.2,

$$
\begin{aligned}
p(y, \xi) & =F_{2} c(y, \xi)=\int e^{-i \xi z} c(y, z) d z=\int e^{-i \xi z} \int e^{i z \zeta} a(y-z, \zeta)^{*} d \zeta d z= \\
& =\int e^{-i z(\xi-\zeta)} a(y-z, \zeta)^{*} d \zeta d z=\int e^{-i z \eta} a(y-z, \xi-\eta)^{*} d \eta d z .
\end{aligned}
$$

Observação 4.4. Notemos que, como visto no capítulo $1, C B^{\infty}\left(\mathbb{R}^{2 n}, A\right)$ é um espaço de Fréchet, com as semi-normas

$$
\|F\|_{j}=\sup _{|\alpha| \leq j}\left\|\partial^{\alpha} F\right\|_{\infty}
$$

onde $F \in C B^{\infty}\left(\mathbb{R}^{2 n}, A\right), \quad j \in \mathbb{N}, \quad \alpha$ é um multi-índice e $\left\|\partial^{\alpha} F\right\|_{\infty}=\sup _{(x, \xi) \in \mathbb{R}^{2 n}}\left\|\partial^{\alpha} F(x, \xi)\right\|$.

Temos também que $C B^{\infty}\left(\mathbb{R}^{2 n}, C B^{\infty}\left(\mathbb{R}^{2 n}, A\right)\right)$ é um espaço de Fréchet com as semi-normas 


$$
\|G\|_{j k}=\sup _{i \leq j} \sup _{|\beta| \leq k}\left\|\partial^{\beta} G\right\|_{i}
$$

$G \in C B^{\infty}\left(\mathbb{R}^{2 n}, C B^{\infty}\left(\mathbb{R}^{2 n}, A\right)\right)$, onde

$$
\|G\|_{i}=\sup _{(z, \eta) \in \mathbb{R}^{2 n}}\|G(z, \eta)\|_{i}
$$

lembremos que, para cada $(z, \eta) \in \mathbb{R}^{2 n}, G(z, \eta)$ é uma função em $C B^{\infty}\left(\mathbb{R}^{2 n}, A\right)$.

Aplicaremos no próximo lema os resultados de [1] sobre integrais oscilatórias, para funções tomando valores no espaço de Fréchet $C B^{\infty}\left(\mathbb{R}^{2 n}, A\right)$.

Lema 4.5. Dada $a \in C B^{\infty}\left(\mathbb{R}^{2 n}, A\right)$, se $p(y, \xi)=\int e^{-i z \eta} a(y-z, \xi-\eta)^{*} d z d \eta$, então $p \in C B^{\infty}\left(\mathbb{R}^{2 n}, A\right)$.

Demonstração. Seja $G: \mathbb{R}^{2 n} \rightarrow C B^{\infty}\left(\mathbb{R}^{2 n}, A\right)$ dada por $G(z, \eta)(y, \xi)=a(y-z, \xi-\eta)$. Provemos que $G \in C B^{\infty}\left(\mathbb{R}^{2 n}, C B^{\infty}\left(\mathbb{R}^{2 n}, A\right)\right)$.

Dado $\varepsilon>0$, existe $\delta>0$ tal que, se $\left\|(z, \eta)-\left(z^{\prime}, \eta^{\prime}\right)\right\|<\delta,(\Leftrightarrow \|(y-z, \xi-\eta)-(y-$ $\left.\left.z^{\prime}, \xi-\eta^{\prime}\right) \|<\delta, \quad \forall(y, \xi) \in \mathbb{R}^{2 n}\right)$, então $\left\|\left(\partial^{\alpha} a\right)(y-z, \xi-\eta)-\left(\partial^{\alpha} a\right)\left(y-z^{\prime}, \xi-\eta^{\prime}\right)\right\|<\varepsilon$, pois $\partial^{\alpha} a$ é uniformemente contínua.

Como, para cada $(z, \eta) \in \mathbb{R}^{2 n}, G(z, \eta)$ é uma função de variável $(y, \xi) \in \mathbb{R}^{2 n}$, escrevemos $\partial^{\alpha}(G(z, \eta))$ para denotar a derivada de multi-índice $\alpha$ da função $G(z, \eta) \in$ $C B^{\infty}\left(\mathbb{R}^{2 n}, A\right)$, para $(z, \eta)$ fixado. E, como $\partial^{\alpha}(G(z, \eta))(y, \xi)=\left(\partial^{\alpha} a\right)(y-z, \xi-\eta)$, temos $\left\|\partial^{\alpha}(G(z, \eta))-\partial^{\alpha}\left(G\left(z^{\prime}, \eta^{\prime}\right)\right)\right\|_{\infty}<\varepsilon$. Logo, dados $j \in \mathbb{N}$ e $\varepsilon>0$, existe $\delta>0$ tal que, se $\left\|(z, \eta)-\left(z^{\prime}, \eta^{\prime}\right)\right\|<\delta,\left\|G(z, \eta)-G\left(z^{\prime}, \eta^{\prime}\right)\right\|_{j}<\varepsilon$, e $G$ é contínua.

Além disso, é fácil ver que $\forall j \in \mathbb{N}, \forall(z, \eta) \in \mathbb{R}^{2 n},\|G(z, \eta)\|_{j} \leq\|a\|_{j}$, i.e. $G$ é limitada.

Vejamos agora que $G$ é derivável. Para isto, provemos que $G$ possui derivadas parciais de primeira ordem.

Se $e_{j}=(0, \cdots, 1,0, \cdots, 0) \in \mathbb{R}^{n}$, provemos que

$$
\lim _{t \rightarrow 0} \frac{G\left(z+t e_{j}, \eta\right)-G(z, \eta)}{t}=H(z, \eta), \quad(z, \eta) \in \mathbb{R}^{2 n}
$$


onde

$$
H(z, \eta)(y, \xi)=\lim _{k \rightarrow 0} \frac{a\left(y-z-k e_{j}, \xi-\eta\right)-a(y-z, \xi-\eta)}{k} .
$$

Notemos que $H(z, \eta) \in C B^{\infty}\left(\mathbb{R}^{2 n}, A\right)$.

Consideraremos $t \geq 0$, o caso $t \leq 0$ é análogo.

Dado $(y, \xi) \in \mathbb{R}^{2 n}$, pelo Teorema Fundamental do Cálculo A.14, temos:

$$
\begin{gathered}
\quad \frac{G\left(z+t e_{j}, \eta\right)(y, \xi)-G(z, \eta)(y, \xi)}{t}-H(z, \eta)(y, \xi)= \\
=\frac{a\left(y-z-t e_{j}, \xi-\eta\right)-a(y-z, \xi-\eta)}{t}+\partial_{j} a(y-z, \xi-\eta)= \\
=\int_{[0, t]} \frac{-\partial_{j} a\left(y-z-h e_{j}, \xi-\eta\right)}{t}+\partial_{j} a(y-z, \xi-\eta) d h= \\
=-\int_{[0, t]} \frac{\partial_{j} a\left(y-z-h e_{j}, \xi-\eta\right)-\partial_{j} a(y-z, \xi-\eta)}{t} d h= \\
=\int_{[0, t]} \int_{[0, h]} \frac{\partial_{j}^{2} a\left(y-z-s e_{j}, \xi-\eta\right)}{t} d s d h, \quad h \in[0, t] .
\end{gathered}
$$

Aqui estamos usando a notação $\partial_{j} a(y, \zeta)=\lim _{k \rightarrow 0} \frac{a\left(y+k e_{j}, \xi\right)-a(y, \xi)}{k}$.

Logo, para todo $(y, \xi) \in \mathbb{R}^{2 n}$,

$$
\begin{gathered}
\left\|\frac{G\left(z+t e_{j}, \eta\right)(y, \xi)-G(z, \eta)(y, \xi)}{t}-H(z, \eta)(y, \xi)\right\| \leq \\
\leq \int_{[0, t]} \int_{[0, h]} \frac{\|a\|_{2}}{t} d s d h \leq t\|a\|_{2},
\end{gathered}
$$

o que implica que, para qualquer $(z, \eta) \in \mathbb{R}^{2 n}$,

$$
\sup _{(y, \xi) \in \mathbb{R}^{2 n}}\left\|\frac{G\left(z+t e_{j}, \eta\right)(y, \xi)-G(z, \eta)(y, \xi)}{t}-H(z, \eta)(y, \xi)\right\| \leq t\|a\|_{2} .
$$

Aplicando o mesmo resultado para $\partial^{\alpha}(G(z, \eta)), \quad|\alpha| \leq j$, a derivada de ordem $\alpha$ da função $G(z, \eta)$, temos:

$$
\begin{gathered}
\lim _{t \rightarrow 0}\left\|\frac{G\left(z+t e_{j}, \eta\right)-G(z, \eta)}{t}-H(z, \eta)\right\|_{j} \leq \\
\leq \lim _{t \rightarrow 0} \sup _{|\alpha| \leq j}\left\|\frac{\partial^{\alpha}\left(G\left(z+t e_{j}, \eta\right)\right)-\partial^{\alpha}(G(z, \eta))}{t}-\partial^{\alpha}(H(z, \eta))\right\|_{\infty} \leq
\end{gathered}
$$




$$
\leq \lim _{t \rightarrow 0} \sup _{|\alpha| \leq j}\left\|\partial^{\alpha} a\right\|_{2} \cdot t=0, \quad \forall j \in \mathbb{N} .
$$

Do mesmo modo, vemos que existem todas as derivadas parciais de $G$ e portanto $G$ é $C^{\infty}$. Como antes, vemos que estas derivadas são limitadas e, portanto, $G \in C B^{\infty}\left(\mathbb{R}^{2 n}, C B^{\infty}\left(\mathbb{R}^{2 n}, A\right)\right)$.

Assim, pela definição de integral oscilatória ([1, (1.3)]),

$$
\int e^{i z \eta} G(z, \eta) d z d \eta \in C B^{\infty}\left(\mathbb{R}^{2 n}, A\right)
$$

e, portanto, $\left(\int e^{i z \eta} G(z, \eta) d z d \eta\right)^{*} \in C B^{\infty}\left(\mathbb{R}^{2 n}, A\right)$, ou seja

$$
p(y, \xi)=\int e^{-i z \eta} a(y-z, \xi-\eta)^{*} d z d \eta \in C B^{\infty}\left(\mathbb{R}^{2 n}, A\right)
$$

Proposição 4.6. Dada $a \in C B^{\infty}\left(\mathbb{R}^{2 n}, A\right)$, existe $p \in C B^{\infty}\left(\mathbb{R}^{2 n}, A\right)$ tal que, se $u, v \in$ $S^{A}\left(\mathbb{R}^{n}\right),<O(a) u, v>=<u, O(p) v>$.

Demonstração. Seja $p(y, \xi)=\int e^{-i z \eta} a(y-z, \xi-\eta)^{*} d z d \eta$. Vimos no lema 4.5 que $p \in C B^{\infty}\left(\mathbb{R}^{2 n}, A\right)$. A integral acima é uma integral oscilatória.

Sejam $\psi_{m}, \psi_{k}^{\prime} \in C_{c}^{\infty}\left(\mathbb{R}^{n}\right)$, como no capítulo 1 e sejam $a_{m, k}(x, w)=\psi_{m}(x) \psi_{k}^{\prime}(w) a(x, w)$ e $p_{m, k}(y, \xi)=\int e^{-i z \eta} a_{m, k}(y-z, \xi-\eta)^{*} d z d \eta$. Segue da proposição 1.6 de [1] que, para cada $(y, \xi) \in \mathbb{R}^{2 n}, \lim _{m, k} p_{m, k}(y, \xi)=p(y, \xi)$.

Dadas $u, v \in S^{A}\left(\mathbb{R}^{n}\right)$,

$$
\begin{gathered}
<u, O(p) v>=\int u(y)^{*}\left[\int e^{i y \xi} p(y, \xi) \hat{v}(\xi) d \xi\right] d y= \\
=\int u(y)^{*}\left[\int e^{i y \xi} \lim _{m, k} p_{m, k}(y, \xi) \hat{v}(\xi) d \xi\right] d y
\end{gathered}
$$

É fácil ver que decorre da desigualdade básica 1.4 de [1] (e da definição de $\psi_{m}$ e $\left.\psi_{k}^{\prime}\right)$ que existem $j \in \mathbb{N}$ e uma constante $M \in \mathbb{R}^{+}$, que não depende de a nem de $\psi_{m}$ ou $\psi_{k}^{\prime}$ (é possível escolher $\psi_{m}$ tais que $\sup _{\alpha, m}\left|\psi_{m}^{(\alpha)}\right|<\infty$, e analogamente para $\psi_{k}^{\prime}$ ), tais que $\left\|p_{m k}(y, \xi)\right\| \leq M\|a\|_{2 j}$.

Assim, $\left\|u(y)^{*} p_{m, k}(y, \xi) \hat{v}(\xi)\right\| \leq M\|a\|_{2 j}\|u(y)\|\|\hat{v}(\xi)\| \in L^{1}\left(\mathbb{R}^{2 n}\right)$. 
Pelo Teorema de Fubini (A.15) temos:

$$
\int u(y)^{*}\left[\int e^{i y \xi} p_{m, k}(y, \xi) \hat{v}(\xi) d \xi\right] d y=\iint u(y)^{*} e^{i y \xi} p_{m, k}(y, \xi) \hat{v}(\xi) d \xi d y .
$$

Pelo Teorema da Convergência Dominada (A.7), temos:

$$
\begin{gathered}
\lim _{m, k} \iint u(y)^{*} e^{i y \xi} p_{m, k}(y, \xi) \hat{v}(\xi) d \xi d y=\iint u(y)^{*} e^{i y \xi} \lim _{m, k} p_{m, k}(y, \xi) \hat{v}(\xi) d \xi d y= \\
=\int u(y)^{*}\left[\int e^{i y \xi} \lim _{m, k} p_{m, k}(y, \xi) \hat{v}(\xi) d \xi\right] d y
\end{gathered}
$$

(novamente pelo Teorema de Fubini).

Portanto, $<u, O(p) v>=\lim _{m, k}<u, O\left(p_{m, k}\right) v>$.

Pelo lema 4.2 e pela observação $4.3,<O\left(a_{m, k}\right) u, v>=<u, O\left(p_{m, k}\right) v>$. Além disso, como $a_{m, k}(x, \xi)=\psi_{m}(x) \psi_{k}^{\prime}(\xi) a(x, \xi)$, temos $\left\|a_{m, k}(x, \xi)\right\| \leq\|a(x, \xi)\|, \forall m, \forall k$. Portanto, $\left\|\hat{u}(\xi)^{*} a(x, \xi)^{*} v(x)\right\| \leq\|a\|_{\infty}\|\hat{u}(\xi)\|\|v(x)\| \in L^{1}\left(\mathbb{R}^{2 n}\right)$.

Aplicando Fubini e o Teorema da Convergência Dominada, temos

$$
\begin{aligned}
& \lim _{m, k}< O\left(a_{m, k}\right) u, v>=\lim _{m, k} \int\left[\int e^{-i x \xi} \hat{u}(\xi)^{*} a_{m, k}(x, \xi)^{*} d \xi\right] v(x) d x= \\
&=\int\left[\int e^{-i x \xi} \hat{u}(\xi)^{*} a(x, \xi)^{*} d \xi\right] v(x) d x=<O(a) u, v>.
\end{aligned}
$$

Ou seja, $<O(a) u, v>=<u, O(p) v>$.

Proposição 4.7. Dada $a \in C B^{\infty}\left(\mathbb{R}^{2 n}, A\right), O(a)$ é Heisenberg-suave.

\section{Demonstração.}

Seja $T=a(x, D)$ e $T_{z, \zeta}=E_{z, \zeta}^{-1} T E_{z, \zeta}$ (como no capítulo 1$)$. Vemos que o símbolo de $T_{z, \zeta}$ é $a_{z, \zeta}, \operatorname{com} a_{z, \zeta}(x, \xi)=a(x+z, \xi+\zeta)$, (da mesma maneira como no caso $A=\varnothing$ $[2,2.20])$. Queremos provar que a aplicação $(z, \zeta) \mapsto T_{z, \zeta}$ é $C^{\infty}$.

Vejamos que $(z, \zeta) \mapsto T_{z, \zeta}$ é contínua em $\left(z_{0}, \zeta_{0}\right)$, i.e., que $\forall \varepsilon>0, \exists \delta>0$, tal que, se $\left\|(z, \zeta)-\left(z_{o} \cdot \zeta_{o}\right)\right\|<\delta$, então $\left\|T_{z, \zeta}-T_{z_{0} \cdot \zeta_{0}}\right\|<\varepsilon$.

Vimos no capítulo 3 (3.19) que existe uma constante $k \in \mathbb{R}^{+}$, independente de $a$, tal que $\|O(a)\| \leq k \pi(a)$, onde $\pi(a)=\sup \left\{\left\|\partial_{x}^{\beta} \partial_{\xi}^{\gamma} a\right\|_{\infty}, \quad \beta, \gamma \leq(1, \cdots, 1)\right\}$. 
Como $T_{z, \zeta}-T_{z_{0}, \zeta_{0}}$ tem símbolo $a_{z, \zeta}-a_{z_{0}, \zeta_{0}}$, temos que $\left\|T_{z, \zeta}-T_{z_{0}, \zeta_{0}}\right\| \leq k \pi\left(a_{z, \zeta}-\right.$ $\left.a_{z_{0}, \zeta_{0}}\right)$.

$\operatorname{Dados} \beta, \gamma \leq(1, \cdots, 1)$,

$$
\begin{gathered}
\left\|\partial_{x}^{\beta} \partial_{\xi}^{\gamma}\left(a_{z, \zeta}-a_{z_{0}, \zeta_{0}}\right)\right\|_{\infty}=\sup _{(x, \xi) \in \mathbb{R}^{2 n}}\left\|\partial_{x}^{\beta} \partial_{\xi}^{\gamma} a_{z, \zeta}(x, \xi)-\partial_{x}^{\beta} \partial_{\xi}^{\gamma} a_{z_{0}, \zeta_{0}}(x, \xi)\right\|= \\
=\sup _{(x, \xi) \in \mathbb{R}^{2 n}}\left\|\left(\partial_{x}^{\beta} \partial_{\xi}^{\gamma} a\right)(x+z . \xi+\zeta)-\left(\partial_{x}^{\beta} \partial_{\xi}^{\gamma}\right)\left(x+z_{0}, \xi+\zeta_{0}\right)\right\| .
\end{gathered}
$$

Como $\partial_{x}^{\beta} \partial_{\xi}^{\gamma} a$ é uniformemente contínua, $\forall \varepsilon>0, \exists \sigma>0$ tal que, se

$$
\left\|(y, \eta)-\left(y^{\prime}, \eta^{\prime}\right)\right\|<\sigma \Rightarrow\left\|\partial_{x}^{\beta} \partial_{\xi}^{\gamma} a(y, \eta)-\partial_{x}^{\beta} \partial_{\xi}^{\gamma} a\left(y^{\prime}, \eta^{\prime}\right)\right\|<\varepsilon
$$

se

$$
\begin{gathered}
\left\|(z, \zeta)-\left(z_{0}, \zeta_{0}\right)\right\|<\sigma \Rightarrow\left\|(z+x, \zeta+\xi)-\left(z_{0}+x, \zeta_{0}+\xi\right)\right\|<\sigma, \quad \forall(x, \xi) \in \mathbb{R}^{2 n} \Rightarrow \\
\Rightarrow\left\|\left(\partial_{x}^{\beta} \partial_{\xi}^{\gamma} a\right)(x+z, \xi+\zeta)-\left(\partial_{x}^{\beta} \partial_{\xi}^{\gamma} a\right)\left(x+z_{0}, \xi+\zeta_{0}\right)\right\|<\varepsilon, \quad \forall(x, \xi) \in \mathbb{R}^{2 n} .
\end{gathered}
$$

Portanto, $\left\|\partial_{x}^{\beta} \partial_{\xi}^{\gamma}\left(a_{z, \zeta}-a_{z_{0}, \zeta_{0}}\right)\right\|_{\infty}<\varepsilon, \quad$ se $\left\|(z, \zeta)-\left(z_{0}, \zeta_{0}\right)\right\|<\sigma$.

Notemos que $\sigma$ depende de $\beta$ e $\gamma$. Como $\beta, \gamma$ são finitos, tomamos $\delta>0$ como sendo o mínimo dos $\sigma$ acima e temos que $\forall \varepsilon>0, \exists \delta>0$ tal que, $\left\|(z, \zeta)-\left(z_{0}, \zeta_{0}\right)\right\|<$ $\delta \Rightarrow \pi\left(a_{z, \zeta}-a_{z_{0}, \zeta_{0}}\right)<\varepsilon$. Logo, $\left\|T_{z, \zeta}-T_{z_{0}, \zeta_{0}}\right\|<\varepsilon$ e $(z, \zeta) \mapsto T_{z, \zeta}$ é contínua em $\mathbb{R}^{2 n}$.

Por outro lado, se $\left\{e_{i}\right\}$ é a base canônica de $\mathbb{R}^{n}$, seja

$$
\partial_{i} T_{z, \zeta}=\lim _{h \rightarrow 0} \frac{T_{z+h e_{i}, \zeta}-T_{z, \zeta}}{h}=\lim _{h \rightarrow 0} \frac{O\left(a_{z+h e_{i}, \zeta}\right)-O\left(a_{z, \zeta}\right)}{h} .
$$

Provemos que $\partial_{i} T_{z, \zeta}=O\left(\partial_{i} a_{z, \zeta}\right)$, onde $\partial_{i} a_{z, \zeta}(x, \xi)=\lim _{h \rightarrow 0} \frac{a_{z, \zeta}\left(x+h e_{i}, \xi\right)-a_{z, \zeta}(x, \xi)}{h}$ e $a_{z, \zeta}(x, \xi)=a(x+z, \xi+\zeta)$.

Temos, como antes, que

$$
\begin{gathered}
\left\|\frac{T_{z+h e_{i}, \zeta}-T_{z, \zeta}}{h}-O\left(\partial_{i} a_{z, \zeta}\right)\right\|=\left\|\frac{O\left(a_{z+h e_{i}, \zeta}-a_{z, \zeta}-h \partial_{i} a_{z, \zeta}\right)}{h}\right\| \\
\leq \frac{k}{h} \pi\left(a_{z+h e_{i}, \zeta}-a_{z, \zeta}-h \partial_{i} a_{z, \zeta}\right) .
\end{gathered}
$$


Dados $(x, \xi) \in \mathbb{R}^{2 n}, h \geq 0$ (o caso $h \leq 0$ é análogo), pelo Teorema Fundamental do Cálculo, (A.14),

$$
\begin{gathered}
a\left(x+h e_{i}+z, \xi+\zeta\right)-a(x+z, \xi+\zeta)-h \partial_{i} a(x+z, \xi+\zeta)= \\
=\int_{[0, h]} \partial_{i} a\left(x+z+t e_{i}, \xi+\zeta\right) d t-h \partial_{i} a(x+z, \xi+\zeta)= \\
=\int_{[0, h]}\left[\partial_{i} a\left(x+z+t e_{i}, \xi+\zeta\right)-\partial_{i} a(x+z, \xi+\zeta)\right] d t= \\
=\int_{[0, h]} \int_{[0, t]} \partial_{i}\left(\partial_{i} a\right)\left(x+z+s e_{i}, \xi+\zeta\right) d s d t= \\
=\int_{[0, h]} \int_{[0, t]} \partial_{i}^{2} a\left(x+z+s e_{i}, \xi+\zeta\right) d s d t .
\end{gathered}
$$

A última igualdade determina o significado da notação $\partial_{i}^{2} a$.

Logo,

$$
\begin{gathered}
\left\|a\left(x+h e_{i}+z, \xi+\zeta\right)-a(x+z, \xi+\zeta)-h \partial_{i} a(x+x, \xi+\zeta)\right\| \leq \\
\leq h^{2} \sup _{(x, \xi) \in \mathbb{R}^{2 n}}\left\|\partial_{i}^{2} a_{z, \zeta}(x, \xi)\right\| .
\end{gathered}
$$

De modo análogo, dados $\beta, \gamma \leq(1,1, \cdots, 1)$, temos que

$$
\begin{gathered}
\left.\|\left(\partial_{x}^{\beta} \partial_{\xi}^{\gamma} a\right)\left(x+z+h e_{i}, \xi+\zeta\right)-\partial_{x}^{\beta} \partial_{\xi}^{\gamma} a\right)(x+z, \xi+\zeta)- \\
-h\left(\partial_{x}^{\beta} \partial_{\xi}^{\gamma} a\right)(x+z, \xi+\zeta) \| \leq \\
h^{2} \sup _{(x, \xi) \in \mathbb{R}^{2 n}}\left\|\partial_{i}^{2} \partial_{x}^{\beta} \partial_{\xi}^{\gamma} a_{z, \zeta}(x, \xi)\right\| \leq h^{2} \pi\left(\partial_{i}^{2} a_{z, \zeta}\right) .
\end{gathered}
$$

Portanto,

$$
\lim _{h \rightarrow 0}\left\|\frac{O\left(a_{z+h e_{i}, \zeta}\right)-O\left(a_{z, \zeta}\right)}{h}-O\left(\partial_{i} a_{z, \zeta}\right)\right\| \leq \lim _{h \rightarrow 0} k \cdot h \pi\left(\partial_{i}^{2} a_{z, \zeta}\right)=0 .
$$

ou seja, $\partial_{i} T_{z, \zeta}=O\left(\partial_{i} a_{z, \zeta}\right)$.

Do mesmo modo, temos que $\partial_{z}^{\beta} \partial_{\zeta}^{\gamma} T_{z, \zeta}=O\left(\partial_{z}^{\beta} \partial_{\zeta}^{\gamma} a_{z, \zeta}\right)$, e temos, como antes, que $(z, \zeta) \mapsto \partial_{z}^{\beta} \partial_{\zeta}^{\gamma} T_{z, \zeta}$ é contínua, i.e., $(z, \zeta) \mapsto T_{z, \zeta}$ é $C^{\infty}$.

Corolário 4.8. Dada $a \in C B^{\infty}\left(\mathbb{R}^{2 n}, A\right), \quad O(a) \in \mathcal{H S}$. 
Nosso objetivo agora é provar que, se vale 4.1, dado um operador $T \in \mathcal{H} \mathcal{S}$ tal que para toda $G \in C B^{\infty}\left(\mathbb{R}^{n}, A\right)$ comuta com $R_{G}$, então existe $F \in C B^{\infty}\left(\mathbb{R}^{n}, A\right)$ de modo que $T=L_{F}$.

$\mathrm{Na}$ verdade, provaremos o resultado acima com uma hipótese mais fraca, que o operador $T \in \mathcal{H S}$ comuta com $R_{g}$, para toda $g \in S^{A}\left(\mathbb{R}^{n}\right)$.

Consideraremos primeiramente o caso $n=1$.

Lema 4.9. Dada $f \in C B^{\infty}(\mathbb{R}, A)$ temos que

$$
\int \gamma(t)\left(1-\partial_{t}\right)^{2} f(t) d t=f(0)
$$

onde

$$
\gamma(t)= \begin{cases}t e^{-t} & \text { se } t \geq 0 \\ 0 & \text { se } t<0\end{cases}
$$

Demonstração. Como $f \in C B^{\infty}(\mathbb{R}, A), \gamma(t)\left(1-\partial_{t}\right)^{2} f(t) \in L^{1}(\mathbb{R}, A)$.

$$
\int \gamma(t)\left(1-\partial_{t}\right)^{2} f(t) d t=\int_{\mathbb{R}^{+}} t e^{-t}\left(f(t)-2 \partial_{t} f(t)+\partial_{t}^{2} f(t)\right) d t .
$$

Fazendo integração por partes (ver A.15), para $R \in \mathbb{R}^{+}$,

$$
\begin{gathered}
\int_{[0, R]} t e^{-t} \partial_{t} f(t) d t=R e^{-R} f(R)-\int_{[0, R]} \partial_{t}\left(t e^{-t}\right) f(t) d t \\
\int_{[0, R]} t e^{-t} \partial_{t}^{2} f(t) d t=R e^{-R} \partial_{t} f(R)-\int_{[0, R]} \partial_{t}\left(t e^{-t}\right) \partial_{t} f(t) d t= \\
=R e^{-R} \partial_{t} f(R)-\left[\left(e^{-R}-R e^{-R}\right) f(R)-f(0)-\int_{[0, R]} \partial_{t}^{2}\left(t e^{-t}\right) f(t) d t\right] .
\end{gathered}
$$

Assim, como $\int_{R^{+}} t e^{-t}\left(1-\partial_{t}\right)^{2} f(t) d t=\lim _{R \rightarrow \infty} \int_{[0, R]} t e^{-t}\left(1-\partial_{t}\right)^{2} f(t) d t$, pela observação A.6, temos:

$$
\int \gamma(t)\left(1-\partial_{t}\right)^{2} f(t) d t=\int_{R^{+}}\left[t e^{-t}+2 \partial_{t}\left(t e^{-t}\right)+\partial_{t}^{2}\left(t e^{-t}\right)\right] f(t) d t+f(0)=f(0) .
$$


Nota 4.10. Para o caso geral, consideramos $\bar{\gamma}(x)=\prod_{j=1}^{n} \gamma\left(x_{j}\right)$, onde $x=\left(x_{1}, x_{2}, \cdots, x_{n}\right)$ e temos, de modo análogo, que $\int \bar{\gamma}(x) \prod_{j=1}^{n}\left(1-\partial_{x_{j}}\right)^{2} f(x) d x=f(0)$, se $f \in C B^{\infty}\left(\mathbb{R}^{n}, A\right)$. De fato, pelo Teorema de Fubini (A.13), temos

$$
\begin{gathered}
\int \bar{\gamma}(x) \prod_{j=1}^{n}\left(1-\partial_{x_{j}}\right)^{2} f(x) d x= \\
=\int \prod_{j=2}^{n} \gamma\left(x_{j}\right)\left(\int \gamma\left(x_{1}\right)\left(1-\partial_{x_{1}}\right)^{2}\left[\prod_{j=2}^{n}\left(1-\partial_{x_{j}}\right)^{2} f\left(x_{1}, x_{2}, \cdots, x_{n}\right)\right] d x_{1}\right) d x_{2} \cdots d x_{n}= \\
=\int \prod_{j=3}^{n} \gamma\left(x_{j}\right)\left(\int \gamma_{x_{2}}\left(1-\partial_{x_{2}}\right)^{2}\left[\prod_{j=3}^{n}\left(1-\partial_{x_{j}}\right)^{2} f\left(0, x_{2}, \cdots, x_{n}\right)\right] d x_{2}\right) d x_{3} \cdots d x_{n}= \\
=\cdots=f(0, \cdots, 0)=f(0) .
\end{gathered}
$$

A proposição seguinte, para o caso $A=\varnothing$, foi provada por Cordes em $[\mathbf{2}$, corolário $2.4]$.

Proposição 4.11. Dadas $a \in C B^{\infty}\left(\mathbb{R}^{2}, A\right)$ e $b=\left(1+\partial_{y}\right)^{2}\left(1+\partial_{\eta}\right)^{2} a$ então temos que

$$
a(x, \xi)=\int \gamma(y) \gamma(\eta) b(x-y, \xi-\eta) d y d \eta \text {. }
$$

Demonstração. Notemos que

$b(x-y, \xi-\eta)=\left[\left(1+\partial_{y}\right)^{2}\left(1+\partial_{\eta}\right)^{2} a\right](x-y, \xi-\eta)=\left(1-\partial_{y}\right)^{2}\left(1-\partial_{\eta}\right)^{2}[a(x-y, \xi-\eta)]$. Logo,

$$
\int \gamma(y) \gamma(\eta) b(x-y, \xi-\eta) d y d \eta=\int \gamma(y) \gamma(\eta)\left(1-\partial_{y}\right)^{2}\left(1-\partial_{\eta}\right)^{2} a(x-y, \xi-\eta) d y d \eta .
$$

Como $\gamma(y) \gamma(\eta)\left(1-\partial_{y}\right)^{2}\left(1-\partial_{\eta}\right)^{2} a(x-y, \xi-\eta) \in L^{1}\left(\mathbb{R}^{2}, A\right)$, podemos aplicar o Teorema de Fubini e, considerando que $\left(1-\partial_{\eta}\right)^{2} a(x-y, \xi-\eta) \in C B^{\infty}(\mathbb{R}, A)$ para $x, \xi, \eta$ fixados, aplicamos o lema 4.9:

$$
\int \gamma(\eta) \int \gamma(y)\left(1-\partial_{y}\right)^{2}\left(1-\partial_{\eta}\right)^{2} a(x-y, \xi-\eta) d y d \eta=\int \gamma(\eta)\left(1-\partial_{y}\right)^{2} a(x, \xi-\eta) d \eta \text {. }
$$

Novamente, para $x, \xi$ fixos, pelo lema $4.9, \int \gamma(\eta)\left(1-\partial_{\eta}\right)^{2} a(x, \xi-\eta) d \eta=a(x, \xi)$ 
Corolário 4.12. Nas hipóteses da proposição 4.11, temos

$$
a(z, \zeta)=\int \gamma(-x) \gamma(-\xi) b(x+z, \xi+\zeta) d x d \xi
$$

Nota 4.13. Para o caso geral, dada $a \in C B^{\infty}\left(\mathbb{R}^{2 n}, A\right)$, seja $b=\prod_{j=1}^{n}\left(1+\partial_{y_{j}}\right)^{2}\left(1+\partial_{\eta_{j}}\right)^{2} a$, e temos, de modo análogo, que $a(z, \zeta)=\int \bar{\gamma}(-x) \bar{\gamma}(-\xi) b(x+z, \xi+\zeta) d x d \xi$, usando a notação da nota 4.10 .

Lema 4.14. Sejam $F$ a transformada de Fourier e $E_{z, \zeta}$ definido como no capítulo 1. Então temos:

1. $F E_{z, \zeta}=\left(E_{-\zeta, z}\right)^{-1} F$

2. $F\left(E_{z, \zeta}\right)^{-1}=E_{-\zeta, z} F$.

\section{Demonstração.}

Dada $u \in S^{A}\left(\mathbb{R}^{n}\right)$,

$$
\begin{gathered}
\text { 1. } F E_{z, \zeta} u(x)=\int e^{-i x y} E_{z, \zeta} u(y) d y=\int e^{-i x y} e^{i \zeta y} u(y-z) d y= \\
=\int e^{-i(y+z)(x-\zeta)} u(y) d y=e^{-i z(x-\zeta)} F u(x-\zeta)=T_{\zeta} M_{-z} F u(x)=\left(E_{-\zeta, z}\right)^{-1} F u(x) . \\
\text { 2. } F E_{z, \zeta}^{-1} u(x)=\int e^{-i x y} T_{-z} M_{-\zeta} u(y) d y=\int e^{-i x y} e^{-i \zeta(y+z)} u(y+z) d y= \\
\int e^{-i x(y-z)} e^{-i \zeta y} u(y) d y=e^{i x z} \int e^{-i y(x+\zeta)} u(y) d y=e^{i x z} F u(x+\zeta)=M_{z} T_{-\zeta} F u(x)= \\
E_{-\zeta, z} F u(x) .
\end{gathered}
$$

Observação 4.15. Lembremos que, como vimos na demonstração da proposição 2.5 , dadas $g, u \in S^{A}\left(\mathbb{R}^{n}\right)$, temos que $R_{g} u(x)=\int e^{i x \xi} F u(\xi) g(x+J \xi) d \xi$

Lema 4.16. Dada $g \in S\left(\mathbb{R}^{n}\right)$, vale que

1. $E_{z, \zeta}^{-1} R_{g}=R_{T_{-z-J \zeta} g} E_{z, \zeta}^{-1}$ 
2. $E_{z, \zeta} R_{g}=R_{T_{z+J \zeta}} E_{z, \zeta}$.

\section{Demonstração.}

1. Dada $u \in S\left(\mathbb{R}^{n}\right)$, pelo lema 4.14 ,

$$
\begin{gathered}
E_{z, \zeta}^{-1} R_{g} u(x)=e^{-i \zeta(x+z)} R_{g} u(x+z)=e^{-i \zeta(x+z)} \int e^{i(x+z) \xi} F u(\xi) g(x+z+J \xi) d \xi \\
R_{T_{-z-J \zeta}} E_{z, \zeta}^{-1} u(x)=\int e^{i x \xi} F\left(E_{z, \zeta}^{-1} u\right)(\xi) T_{-z-J \zeta} g(x+J \xi) d \xi= \\
=\int e^{i x \xi} E_{-\zeta, z} F u(\xi) g\left(x+z+J(\xi+\zeta) d \xi=\int e^{i x \xi} e^{i z \xi} F u(\xi+\zeta) g(x+z+J(\xi+\zeta)) d \xi=\right. \\
=\int e^{i(x+z)(\xi-\zeta)} F u(\xi) g(x+z+J \xi) d \xi=E_{z, \zeta}^{-1} R_{g} u(x) . \\
E_{z, \zeta} R_{g} u(x)=e^{i \zeta x} R_{g} u(x-z)=e^{i \zeta x} \int e^{i(x-z) \xi} F u(\xi) g(x-z+J \zeta) d \xi . \\
R_{T_{z+J \zeta} g} E_{z, \zeta} u(x)=\int e^{i x \xi} F\left(E_{z, \zeta} u\right)(\xi) T_{z+J \zeta} g(x+J \xi) d \xi= \\
=\int e^{i x \xi}\left(E_{-\zeta, z}\right)^{-1} F u(\xi) g(x-z+J(\xi-\zeta)) d \xi=\int e^{i x \xi} e^{-i z(\xi-\zeta)} F u(\xi-\zeta) g(x-z+J(\xi-\zeta)) d \xi= \\
=\int e^{i x(\xi+\zeta)} e^{-i z \xi} F u(\xi) g(x-z+J \xi) d \xi=E_{z, \zeta} R_{g} u(x) .
\end{gathered}
$$

Proposição 4.17. Dado $T \in \mathcal{B}^{*}\left(\overline{S^{A}\left(\mathbb{R}^{n}\right)}\right)$, se $\left[T, R_{g}\right]=0, \forall g \in S^{A}\left(\mathbb{R}^{n}\right)$, então $\left[T_{z, \zeta}, R_{g}\right]=$ $0, \forall g \in S^{A}\left(\mathbb{R}^{n}\right)$.

Demonstração. Pelo lema 4.16, temos $T_{z, \zeta} R_{g}=E_{z, \zeta}^{-1} T E_{z, \zeta} R_{g}=E_{z, \zeta}^{-1} T R_{T_{z+J \zeta} g} E_{z, \zeta}=E_{z, \zeta}^{-1} R_{T_{z+J \zeta} g} T E_{z, \zeta}=R_{g} E_{z, \zeta}^{-1} T E_{z, \zeta}=R_{g} T_{z, \zeta}$.

Proposição 4.18. Dadas $a \in C B^{\infty}\left(\mathbb{R}^{2 n}, A\right)$ e $b=\prod_{j=1}^{n}\left(1+\partial_{x_{j}}\right)^{2}\left(1+\partial_{\xi_{j}}\right)^{2} a$, se $O(a) \in \mathcal{H} \mathcal{S}$ e $\left[O(a), R_{g}\right]=0$, para todo $g \in S^{A}\left(\mathbb{R}^{n}\right)$, então $O(b) \in \mathcal{H S}$ e $\left[O(b), R_{g}\right]=0, \forall g \in$ $S^{A}\left(\mathbb{R}^{n}\right)$. 
Demonstração. Notemos que, como visto na demonstração de 4.7, se $T=O(a)$ e $B=O(b), B_{z, \zeta}=\prod_{j=1}^{n}\left(1+\partial_{z_{j}}\right)^{2}\left(1+\partial_{\zeta_{j}}\right)^{2} T_{z, \zeta}$

Logo, se $(z, \zeta) \mapsto T_{z, \zeta}$ é $C^{\infty},(z, \zeta) \mapsto B_{z, \zeta}$ é $C^{\infty}$.

Vimos na proposição 4.17 que se $\left[T, R_{g}\right]=0, \forall g \in S^{A}\left(\mathbb{R}^{n}\right)$, então $\left[T_{z, \zeta}, R_{g}\right]=$ $0, \forall g \in S^{A}\left(\mathbb{R}^{n}\right)$. Assim, se $\left\{e_{j}\right\}$ é a base canônica de $\mathbb{R}^{n}$,

$$
\begin{gathered}
\left(\partial_{z_{j}} T_{z, \zeta}\right) R_{g}=\lim _{h \rightarrow 0} \frac{T_{z+h e_{j}, \zeta}-T_{z, \zeta}}{h} R_{g}= \\
\lim _{h \rightarrow 0} \frac{T_{z+h e_{j}, \zeta} R_{g}-T_{z, \zeta} R_{g}}{h}=\lim _{h \rightarrow 0} R_{g} \frac{T_{z+h e_{j}, \zeta}-T_{z, \zeta}}{h}=R_{g}\left(\partial_{z_{j}} T_{z, \zeta}\right) .
\end{gathered}
$$

De modo análogo, provamos que $\left[R_{g}, B\right]=0, \forall g \in S^{A}\left(\mathbb{R}^{n}\right)$.

Teorema 4.19. Dado $T \in \mathcal{B}^{*}\left(\overline{S^{A}\left(\mathbb{R}^{n}\right)}\right)$, Heisenberg-suave, tal que para toda $g \in$ $S^{A}\left(\mathbb{R}^{n}\right),\left[T, R_{g}\right]=0$. Supondo que vale 4.1 , então existe $F \in C B^{\infty}\left(\mathbb{R}^{n}, A\right)$ de modo que $T=L_{F}$.

Demonstração. Por 4.1 temos que existe $a \in C B^{\infty}\left(\mathbb{R}^{2 n}, A\right)$ de modo que $T=O(a)$. Sejam $b=\prod_{j=1}^{n}\left(1+\partial_{x_{j}}\right)^{2}\left(1+\partial_{\xi_{j}}\right)^{2} a$ e $B=O(b)$. Pela proposição 4.18, temos que $\left[B, R_{g}\right]=0, \forall g \in S^{A}\left(\mathbb{R}^{n}\right)$. Logo, $\left[B_{z, \zeta}, R_{g}\right]=0, \forall g \in S^{A}\left(\mathbb{R}^{n}\right)$, pela proposição 4.17 . Assim, pelo visto na demonstração do teorema $2.12, B_{z, \zeta}=B_{z-J \zeta, 0}$.

Por outro lado, o símbolo de $B_{z, \zeta}$ é $b(x+z, \xi+\zeta)$ e o símbolo de $B_{z-J \zeta, 0}$ é $b(x+z-J \zeta, \xi)$. Portanto, por 4.1, temos que $b(x+z, \xi+\zeta)=b(x+z-J \zeta, \xi)$. Assim, pela nota 4.13 , temos:

$$
\begin{gathered}
a(z, \zeta)=\int \bar{\gamma}(-x) \bar{\gamma}(-\xi) b(x+z, \xi+\zeta) d x d \xi= \\
=\int \bar{\gamma}(-x) \bar{\gamma}(-\xi) b(x+z-J \zeta, \xi) d x d \xi=a(z-J \zeta, 0) .
\end{gathered}
$$

Tomando $F(z)=a(z, 0)$, temos $F \in C B^{\infty}\left(\mathbb{R}^{n}, A\right)$ e $a(z, \zeta)=F(z-J \zeta)$, e, pela observação 1.8 , portanto, $T=L_{F}$. 


\section{Apêndice A}

Veremos agora alguns resultados sobre integrais de funções que chegam num espaço de Banach, Integral de Bochner. Para isto, nos baseamos fundamentalmente no apêndice E de $[\mathbf{1 3}]$.

Seja $E$ um espaço de Banach separável e seja $\mathcal{B}(E)$ a $\sigma$-álgebra gerada pelos subconjuntos abertos de $E$.

Definição A.1. Dizemos que uma função $f: \mathbb{R}^{n} \rightarrow E$ é mensurável se é mensurável com respeito a $\mathcal{B}\left(\mathbb{R}^{n}\right)$ e a $\mathcal{B}(E)$.

É fácil ver que, se $f$ é mensurável, então $x \mapsto\|f(x)\|$ é mensurável.

Definição A.2. Uma função mensurável $f: \mathbb{R}^{n} \rightarrow E$ é dita simples se $f\left(\mathbb{R}^{n}\right)$ é um subconjnto finito de $E$. Na verdade, se $f\left(\mathbb{R}^{n}\right)=\left\{a_{1}, \cdots, a_{p}\right\}$, seja $J_{l}=f^{-1}\left(a_{l}\right)$; então, $f(x)=\sum_{l=1}^{p} a_{l} \chi_{J_{l}}(x)$, onde $\chi_{J_{l}}$ é a função característica de $J_{l}$ e onde os conjuntos $J_{l}$ são disjuntos e pertencem a $\mathcal{B}\left(\mathbb{R}^{n}\right)$.

Lema A.3. Dada $f: \mathbb{R}^{n} \rightarrow E$, seja $D \subseteq E$ enumerável denso em $f\left(\mathbb{R}^{n}\right)$. Então, $\forall \varepsilon>0, \forall a \in f\left(\mathbb{R}^{n}\right)$, existem $d \in D$ e $r \in \mathbb{Q}$, tais que $\|r d\| \leq\|a\|$ e $\|r d-a\|<\varepsilon$.

Demonstração. Dado $1>\varepsilon>0$, como $D$ é denso em $f\left(\mathbb{R}^{n}\right)$, existe $d \in D$ tal que $\|a-d\|<\varepsilon$. Seja $r \in \mathbb{Q}^{+}$tal que $1-\varepsilon<r<1$ e $r \leq \frac{\|a\|}{\|d\|}$. Assim, $\|r d\| \leq\|a\|$ e temos

$$
\|r d-a\| \leq\|d-r d\|+\|a-d\| \leq\|d\|(1-r)+\varepsilon \leq \frac{\|a\|}{r} \varepsilon+\varepsilon \leq\|a\| \frac{\varepsilon}{1-\varepsilon}+\varepsilon .
$$


Dado $\varepsilon_{1}>0$, escolhemos $\varepsilon>0$ de modo que $\|a\| \frac{\varepsilon}{1-\varepsilon}+\varepsilon \leq \varepsilon_{1}$ e temos $\|r d-a\|<\varepsilon_{1}$ e $\|d\| \leq\|a\|$

Proposição A.4. Dada $f: \mathbb{R}^{n} \rightarrow E$, mensurável, existe uma seqüência $\left(f_{k}\right)_{k \in N}$ de funções simples tal que $\forall x \in \mathbb{R}^{n}, f(x)=\lim _{k \rightarrow \infty} f_{k}(x)$, e $\left\|f_{k}(x)\right\| \leq\|f(x)\|, \forall k \in \mathbb{N}$.

Demonstração. Suponhamos que $f$ é não nula.

Seja $D \subseteq E$ enumerável, denso em $f\left(\mathbb{R}^{n}\right)$. Seja $D^{\prime}$ o conjunto dos múltiplos racionais de $D, D^{\prime}=\left\{c_{k}\right\}_{k \in \mathbb{N}}$. Podemos assumir que $c_{1}=0$.

Para cada $x \in \mathbb{R}^{n}$, e cada $k \in \mathbb{N}$, seja $E_{k}(x) \subseteq E$ dado por:

$$
E_{k}(x)=\left\{c_{j} \in D^{\prime}, j \leq k \text { e }\left\|c_{j}\right\| \leq\|f(x)\|\right\} .
$$

Como $c_{1}=0, E_{k}(x)$ é não vazio.

Vamos construir a seqüência $\left(f_{k}\right)_{k \in \mathbb{N}}$, tomando $f_{k}(x)$ o elemento de $E_{k}(x)$ que está mais próximo de $f(x)$.

Consideremos

$$
A_{k}(x)=\left\{c_{i} \in E_{k}(x) /\left\|f(x)-c_{i}\right\|=\inf \left\{\left\|f(x)-c_{j}\right\|, c_{j} \in E_{k}(x)\right\}\right\} .
$$

Seja $I_{k}(x)$ o conjunto dos índices $\operatorname{dos} c_{i} \in A_{k}(x)$ e seja $i_{0}=\min I_{k}(x)$ e $f_{k}(x)=c_{i_{0}}$.

Como os conjuntos $\left\{x \in \mathbb{R}^{n}, f_{k}(x)=c_{i_{0}}\right\}$ podem ser descritos por meio de desigualdades envolvendo $\|f(x)\|,\left\|c_{j}\right\|, j=1, \cdots, k$, e $\left\|f(x)-c_{j}\right\|, j=1, \cdots, k$, cada $f_{k}$ é mensurável. É fácil ver, então, que $f_{k}$ é simples, $\forall k \in \mathbb{N}$. Além disso, por construção de $f_{k}$ e de $E_{k}(x)$, temos que $\left\|f_{k}(x)\right\| \leq\|f(x)\|, \forall x \in \mathbb{R}, \forall k \in \mathbb{N}$.

Vejamos agora que $\left(f_{k}\right)_{k \in \mathbb{N}}$ converge pontualmente para $f$. Consideremos:

1. Pelo lema A.3, para todo $\varepsilon>0$, para todo $x \in \mathbb{R}^{n}$, existe $c_{h_{0}} \in D^{\prime}$ tal que $\left\|c_{h_{0}}\right\| \leq\|f(x)\|$ (isto implica $\left.c_{h_{0}} \in E_{h_{0}}(x)\right)$ e $\left\|c_{h_{0}}-f(x)\right\|<\varepsilon$.

2. Se $k \geq h_{0}, E_{h_{0}}(x) \subseteq E_{k}(x)$.

3. $\left\|f(x)-f_{k}(x)\right\| \leq\left\|f(x)-c_{k}\right\|$, para qualquer $c_{k} \in E_{k}(x)$.

Assim, dado $\varepsilon>0$, seja $h_{0} \in \mathbb{N}$ como em (1). Então temos, $\forall k \geq h_{0}, \| f(x)-$ $f_{k}(x)\|\leq\| f(x)-c_{h_{0}} \|<\varepsilon$. 
Definição A.5. Seja $f: \mathbb{R}^{n} \rightarrow E$, mensurável. Dizemos que $f$ é Bochner integrável (integrável) se a função $x \mapsto\|f(x)\|$ é integrável.

Dada $f: \mathbb{R}^{n} \rightarrow E$, simples e integrável, com $f(x)=\sum_{l=1}^{p} a_{l} \chi_{J_{l}}, a_{l} \in E$ e $J_{l} \in$ $\mathcal{B}\left(\mathbb{R}^{n}\right)$, definimos a integral de $f$ como segue:

$$
\int f(x) d x=\sum_{l=1}^{p} a_{l} \mu\left(J_{l}\right)
$$

onde $\mu$ é a medida de Lebesgue em $\mathbb{R}^{n}$. É claro que $f$ integrável implica que $\mu\left(J_{l}\right)<$ $\infty, l=1, \cdots, p$.

É fácil ver que

$$
\left\|\int f(x) d x\right\| \leq \int\|f(x)\| d x
$$

e que, se $\alpha, \beta \in \varnothing$ e $g$ uma função simples integrável,

$$
\int(\alpha f+\beta g)(x) d x=\alpha \int f(x) d x+\beta \int g(x) d x .
$$

Dada $f: \mathbb{R}^{n} \rightarrow E$ integrável, seja $\left(f_{k}\right)_{k \in \mathbb{N}}$ uma seqüência de funções simples tal que $f(x)=\lim _{k \rightarrow \infty} f_{k}(x), \forall x \in \mathbb{R}^{n}$ e tal que $x \mapsto \sup _{k}\left\|f_{k}(x)\right\|=\|f(x)\|$ é integrável (ver A.4). Como $\left\|f_{k}(x)-f(x)\right\| \leq 2\|f(x)\|$, pelo Teorema da Convergência Dominada, $\int\left\|f_{k}(x)-f(x)\right\| d x \underset{x \rightarrow \infty}{\longrightarrow} 0$. Logo, a seqüência $\left(\int f_{k}\right)_{k \in \mathbb{N}}$ é de Cauchy em $E$ e portanto converge.

Definimos

$$
\int f(x) d x=\lim _{k \rightarrow \infty} \int f_{k}(x) d x .
$$

É fácil ver que esta definição não depende da escolha da seqüência $\left(f_{k}\right)_{k \in \mathbb{N}}[\mathbf{1 3}$, pg.352].

Proposição A.6. Seja $f: \mathbb{R}^{n} \rightarrow E$, integrável. Então

$$
\left\|\int f(x) d x\right\| \leq \int\|f(x)\| d x
$$


Demonstração. Seja $\left(f_{k}\right)_{k \in \mathbb{N}}$ uma seqüência de funções simples (como na proposição A.5), tal que $\forall x \in \mathbb{R}^{n}, \sup _{k}\left\|f_{k}(x)\right\| \leq\|f(x)\|$ e $f(x)=\lim _{k} f_{k}(x)$.

Logo, $\left\|\int f_{k}(x) d x\right\| \leq \int\left\|f_{k}(x)\right\| d x \leq \int\|f(x)\| d x$. Como $\int f(x) d x=\lim _{k \rightarrow \infty} \int f_{k}(x) d x$, vale a tese.

Teorema da Convergência Dominada A.7. Sejam $f, f_{1}, f_{2}, \cdots$ funções de $\mathbb{R}^{n}$ em $E$, mensuráveis, tais que $f(x)=\lim _{k \rightarrow \infty} f_{k}(x)$ para quase todo $x \in \mathbb{R}^{n}$ e $\left\|f_{k}(x)\right\| \leq g(x)$, q.s., para todo $k \in \mathbb{N}$, onde $g: \mathbb{R}^{n} \rightarrow[0,+\infty]$, integrável. Então, $f, f_{1}, \cdots$ são integráveis e $\int f(x) d x=\lim _{k \rightarrow \infty} \int f_{k}(x) d x$.

Demonstração. Como $\left\|f_{k}(x)\right\| \leq g(x)$ q.s., $\forall k \in \mathbb{N}$, a integrabilidade de $f_{1}, f_{2}, \cdots$ é imediata. Além disso, temos $\|f(x)\| \leq g(x)$ q.s. e $f$ é integrável. Portanto, $\|\left(f_{k}-\right.$ $f)(x) \| \leq 2 g(x)$ q.s.. Pelo Teorema da Convergência Dominada (caso escalar) temos então que $\lim _{k \rightarrow \infty} \int\left\|\left(f_{k}-f\right)(x)\right\| d x=0$, e, pela proposição A.6, temos $\left\|\int\left(f_{k}-f\right)(x) d x\right\| \leq$ $\int\left\|\left(f_{k}-f\right)(x)\right\| d x \underset{k \rightarrow \infty}{\rightarrow} 0$.

Observação A.8. Se $f: \mathbb{R}^{n} \rightarrow E$ é integrável e $J \in \mathcal{B}\left(\mathbb{R}^{n}\right)$, definimos

$$
\int_{J} f(x) d x=\int_{\mathbb{R}^{n}} f(x) \chi_{J}(x) d x
$$

Se $g: \mathbb{R} \rightarrow E$ é integrável, notemos que $\int_{\mathbb{R}} g(t) d t=\lim _{R \rightarrow \infty} \int_{[-R, R]} g(t) d t$, pois, se $\left(R_{k}\right)_{k \in \mathbb{N}}$ é uma seqüência em $\mathbb{R}^{+}$, crescente, com $\lim _{k \rightarrow \infty} R_{k}=+\infty$, e se $g_{k}(t)=g(t) \chi_{\left[-R_{k}, R_{k}\right]}(t)$, temos que, para cada $t \in \mathbb{R}, \lim _{k \rightarrow \infty} g_{k}(t)=g(t)$ e $\sup _{k}\left\|g_{k}(t)\right\| \leq\|g(t)\|$; portanto, pelo Teorema da Convergência Dominada A.7

$$
\int_{\mathbb{R}} g(t) d t=\lim _{k \rightarrow \infty} \int_{\mathbb{R}} g_{k}(t) d t=\lim _{k \rightarrow \infty} \int_{\left[-R_{k}, R_{k}\right]} g(t) d t .
$$

Definição A.9. Seja $\mathcal{L}^{1}\left(\mathbb{R}^{n}, E\right)$ o conjunto das funções de $\mathbb{R}^{n}$ em $E$ integráveis. Definimos

$$
\|f\|_{L^{1}}=\int\|f(x)\| d x, \text { para } f \in \mathcal{L}^{1}\left(\mathbb{R}^{n}, E\right)
$$


É claro que $\|\cdot\|_{L^{1}}$ é semi-norma. É fácil ver que o conjunto $L^{1}\left(\mathbb{R}^{n}, E\right)$ das classes de equivalência de $\mathcal{L}^{1}\left(\mathbb{R}^{n}, E\right)$, com respeito à igualdade quase sempre, é um espaço vetorial normado onde $\|\cdot\|_{L^{1}}$ é a norma ([13, proposição E.4]). Também podemos provar que $L^{1}\left(\mathbb{R}^{n}, E\right)$ é completo adaptando a demonstração de [13, teorema 3.4.1].

De um modo análogo, definimos os espaços de Banach $L^{p}\left(\mathbb{R}^{n}, E\right)$ das classes de equivalência de funções iguais q.s. que tem a propriedade $\int\|f(x)\|^{p} d x<\infty$. A norma é dada por $\|f\|_{L^{p}}=\left(\int\|f(x)\|^{p} d x\right)^{\frac{1}{p}}$, para $f \in L^{p}\left(\mathbb{R}^{n}, E\right)$.

Proposição A.10. As funções simples do tipo $\sum_{l=1}^{q} a_{l} \chi_{J_{l}}, \mu\left(J_{l}\right)<\infty, a_{l} \in E$, são densas em $L^{p}\left(\mathbb{R}^{n}, E\right)$.

Demonstração. É fácil ver, pela definição A.5, que as funções simples do enunciado pertencem a $L^{p}\left(\mathbb{R}^{n}, E\right)$. Dada uma função $f \in L^{p}\left(\mathbb{R}^{n}, E\right)$, seja uma seqüência de funções simples $\left(f_{k}\right)_{k \in \mathbb{N}}$, como na proposição A.4, tal que, para quase todo $x \in \mathbb{R}^{n}$, vale que $f(x)=\lim _{k \rightarrow \infty} f_{k}(x)$ e $\left\|f_{k}(x)\right\| \leq\|f(x)\|$. Assim, $\left\|\left(f_{k}-f\right)(x)\right\|^{p} \leq 2^{p}\|f(x)\|^{p}$. Logo, pelo Teorema da Convergência Dominada, (A.7), $\lim _{k \rightarrow \infty} \int\left\|\left(f_{k}-f\right)(x)\right\|^{p} d x=0$, o que implica $\left\|f-f_{k}\right\|_{L^{p}} \underset{k \rightarrow \infty}{\rightarrow} 0$.

Um resultado útil, mas que não provaremos aqui, é o teorema E.9 de [13], que diz o seguinte:

Teorema A.11. Uma função $f: \mathbb{R}^{n} \rightarrow E$ é mensurável se e somente se, para todo funcional linear contínuo $\varphi$ em $E^{*}$, a função $\varphi \circ f$ é mensurával.

Proposição A.12. Dada $f: \mathbb{R}^{n} \rightarrow E$, integrável, então, para qualquer funcional linear contínuo $\varphi \in E^{*}$ vale que

$$
\int \varphi \circ f(x) d x=\varphi\left(\int f(x) d x\right)
$$

Além disso, se existe $a_{0} \in E$ tal que $\forall \varphi \in E^{*}, \varphi\left(a_{0}\right)=\int \varphi \circ f(x) d x$, então $a_{0}=$ $\int f(x) d x$ 
Demonstração. Dado qualquer $\varphi \in E^{*}$, por (A.11), sabemos que $\varphi \circ f$ é mensurável. Vejamos que $\varphi \circ f$ é integrável.

$$
\int|\varphi \circ f(x)| d x \leq \int\|\varphi\|\|f(x)\| d x=\|\varphi\| \int\|f(x)\| d x .
$$

Como $f$ é integrável, $\varphi \circ f$ é integrável.

Suponhamos que $f$ é simples, $f(x)=\sum_{l=1}^{p} a_{l} \chi_{J_{l}}(x), \quad a_{l} \in E \quad$ e $\mu\left(J_{l}\right)<\infty$, onde $J_{l}$ são disjuntos, $l=1, \cdots, p$.

$$
\begin{gathered}
\varphi\left(\int f(x) d x\right)=\varphi\left(\sum_{l=1}^{p} a_{l} \mu\left(J_{l}\right)\right)=\sum_{l=1}^{p} \varphi\left(a_{l}\right) \mu\left(J_{l}\right) . \\
\int \varphi \circ f(x) d x=\int \varphi\left(\sum_{l=1}^{p} a_{l} \chi_{J_{l}}(x)\right) d x=\int \sum_{l=1}^{p} \varphi\left(a_{l}\right) \chi_{J_{l}}(x) d x=\sum_{l=1}^{p} \varphi\left(a_{l}\right) \mu\left(J_{l}\right) .
\end{gathered}
$$

Portanto, vale $(*)$ para funções simples.

Seja agora $f: \mathbb{R}^{n} \rightarrow E$ integrável e $\left(f_{k}\right)_{k \in \mathbb{N}}$ uma seqüência de funções simples tal que, para quase todo $x \in \mathbb{R}^{n}, f(x)=\lim _{k \rightarrow \infty} f_{k}(x)$ e $\sup _{k}\left\|f_{k}(x)\right\| \leq\|f(x)\|$. Desta maneira, é claro que $f_{k}$ é integrável para todo $k \in \mathbb{N}$ e temos que

$$
\varphi\left(\int f_{k}(x) d x\right)=\int \varphi \circ f_{k}(x) d x .
$$

Além disso, $\left\|f_{k}(x)\right\| \leq\|f(x)\| \in L^{1}\left(\mathbb{R}^{n}\right)$, para quase todo $x \in \mathbb{R}^{n}$; então, pelo Teorema da Convergência Dominada (A.7), $\lim _{k \rightarrow \infty} \int f_{k}(x) d x=\int f(x) d x$ e, portanto,

$$
\lim _{k \rightarrow \infty} \varphi\left(\int f_{k}(x) d x\right)=\varphi\left(\int f(x) d x\right)
$$

Por outro lado, $\left|\varphi \circ f_{k}(x)\right| \leq\|\varphi\|\left\|f_{k}(x)\right\| \leq\|\varphi\|\|f(x)\| \in L^{1}\left(\mathbb{R}^{n}\right)$, para quase todo $x \in \mathbb{R}^{n}$ e $\forall k \in \mathbb{N}$. Como $\lim _{k \rightarrow \infty} \varphi \circ f_{k}(x)=\varphi \circ f(x)$ q.s., pelo Teorema da Convergência Dominada, $\varphi\left(\int f(x) d x\right) \underset{(2)}{=} \lim _{k \rightarrow \infty} \varphi\left(\int f_{k}(x) d x\right)=\lim _{(1)} \int \varphi \circ f_{k}(x) d x=$ $\int \varphi \circ f(x) d x$, e vale $(*)$

Suponhamos que existe $a_{0} \in E$ tal que $\varphi\left(a_{0}\right)=\int \varphi \circ f d x, \forall \varphi \in E^{*}$. Deste modo, temos que $\varphi\left(a_{0}-\int f(x) d x\right)=0, \forall \varphi \in E^{*}$. Logo, pelo Teorema de Hahn- 
Banach, (ver também $\left[\mathbf{1 3}\right.$, corolário E.8]), existe $\psi \in E^{*}$ tal que $\psi\left(a_{0}-\int f(x) d x\right)=$ $\left\|a_{0}-\int f(x) d x\right\|$, ou seja, $\left\|a_{0}-\int f(x) d x\right\|=0$ e portanto $a_{0}=\int f(x) d x$.

Tendo este resultado, podemos ver que várias propriedades que são válidas para integração de funções complexas são válidas também para funções que tomam valores num espaço de Banach separável.

Teorema de Fubini A.13. Se $f \in L^{1}\left(\mathbb{R}^{2 n}, E\right)$, então $y \mapsto f(x, y)$ pertence a $L^{1}\left(\mathbb{R}^{n}, E\right)$ para quase todo $x \in \mathbb{R}^{n}$ e $x \mapsto f(x, y)$ pertence a $L^{1}\left(\mathbb{R}^{n}, E\right)$ para quase todo $y \in$ $\mathbb{R}^{n}$. Além disso, as funções $h(x)=\int f(x, y) d y$ e $k(y)=\int f(x, y) d x$ definidas q.s. pertencem a $L^{1}\left(\mathbb{R}^{n}, E\right)$ e vale:

$$
\int f(x, y) d x d y=\int\left[\int f(x, y) d y\right] d x=\int\left[\int f(x, y) d x\right] d y
$$

Demonstração. Por definição, temos que $\|f\| \in L^{1}\left(\mathbb{R}^{2 n}\right)$. Logo, pelo Teorema de Fubini, [13, (2.37)], temos que, para quase todo $x \in \mathbb{R}^{n}, y \mapsto\|f(x, y)\|$ pertence a $L^{1}\left(\mathbb{R}^{n}\right)$, isto é, $y \mapsto f(x, y) \in L^{1}\left(\mathbb{R}^{n}, E\right)$ q.s.. Analogamente para $x \mapsto f(x, y)$.

Também temos que, se $\bar{h}(x)=\int\|f(x, y)\| d y, \bar{h} \in L^{1}\left(\mathbb{R}^{n}\right)$. E, como $\|h(x)\| \leq$ $\bar{h}(x), h \in L^{1}\left(\mathbb{R}^{n}, E\right)$. Do mesmo modo, $k \in L^{1}\left(\mathbb{R}^{n}, E\right)$.

Por outro lado, dado qualquer $\varphi \in E^{*}$, temos $\varphi \circ f \in L^{1}\left(\mathbb{R}^{2 n}\right)$, pois $\int \| \varphi \circ$ $f(x, y)\|d x d y \leq\| \varphi\left\|\int\right\| f(x, y) \| d x d y<\infty$. Também $\varphi \circ k, \varphi \circ h \in L^{1}\left(\mathbb{R}^{n}\right)$. Então, apliquemos [3, 2.37]:

$$
\begin{aligned}
\int \varphi \circ h(x) d x & =\int\left[\varphi\left(\int f(x, y) d y\right)\right] d x=\int\left[\int \varphi \circ f(x, y) d y\right] d x= \\
& =\int\left[\int \varphi \circ f(x, y) d x\right] d y=\int \varphi \circ k(y) d y .
\end{aligned}
$$

Logo, pela proposição A.12, $\varphi\left(\int h(x) d x\right)=\varphi\left(\int k(y) d y\right), \forall \varphi \in E^{*}$, e então $\int h(x) d x=\int k(y) d y$, ou seja, 


$$
\int\left[\int f(x, y) d y\right] d x=\int\left[\int f(x, y) d x\right] d y .
$$

Do mesmo modo, temos

$$
\begin{gathered}
\int \varphi \circ f(x, y) d x d y=\int \varphi \circ k(y) d y, \forall \varphi \in E^{*} \Rightarrow \\
\Rightarrow \int f(x, y) d x d y=\int k(y) d y=\int\left[\int f(x, y) d x\right] d y
\end{gathered}
$$

Teorema Fundamental do Cálculo A.14. Seja $f:[a, b] \rightarrow E$ uma função contínua e diferenciável tal que $f^{\prime}$ (a função derivada de $f$ ) é limitada. Então:

$$
\int_{[a, b]} f^{\prime}(t) d t=f(b)-f(a)
$$

Demonstração. Dado $\varphi \in E^{*}$, qualquer, consideremos a função $\varphi \circ f:[a, b] \rightarrow C$. Seja $F(t)=\varphi \circ f(t)$. Notemos que

$$
F^{\prime}\left(t_{0}\right)=\lim _{h \rightarrow 0} \frac{F\left(t_{0}+h\right)-F\left(t_{0}\right)}{h}=\lim _{h \rightarrow 0} \varphi\left(\frac{f\left(t_{0}+h\right)-f\left(t_{0}\right)}{h}\right)=\varphi \circ f^{\prime}\left(t_{0}\right) .
$$

Logo, $F$ é contínua, diferenciável, com derivada limitada. Além disso, como $F^{\prime}$ é mensurável, isto é, $\varphi \circ f^{\prime}$ é mensurável para todo $\varphi \in E^{*}$, pelo teorema A.11 temos que $f^{\prime}$ é mensurável. Assim, por $[\mathbf{3},(3.36)]$, temos: $\int_{a}^{b} F^{\prime}(t) d t=F(b)-F(a)$, isto é, $\varphi\left(\int_{[a, b]} f^{\prime}(t) d t\right)=\varphi(f(b)-f(a)), \forall \varphi \in E^{*}$ e, portanto, $\int_{[a, b]} f^{\prime}(t) d t=f(b)-f(a)$.

Corolário A.15. Integração por partes. Dadas $f, g \in C_{c}^{\infty}\left(\mathbb{R}^{n}, E\right)$, então, para $-\infty<a<b<\infty$, vale que

$$
\int_{[a, b]} f(x) g^{\prime}(x) d x+\int_{[a, b]} f^{\prime}(x) g(x) d x=f(b) g(b)-f(a) g(a) .
$$


Demonstração. Notemos que $f g \in C_{c}^{\infty}\left(\mathbb{R}^{n}, E\right)$. Além disso, $(f g)^{\prime}(x)=f(x) g^{\prime}(x)+$ $f^{\prime}(x) g(x)$. Logo, pelo Teorema Fundamental do Cálculo A.14 aplicado à função $f g$,

$$
\int_{[a, b]} f(x) g^{\prime}(x) d x+\int_{[a, b]} f^{\prime}(x) g(x) d x=f(b) g(b)-f(a) g(a) .
$$

Corolário A.16. Seja $A$ uma $C^{*}$-álgebra separável. Dadas $f \in C^{\infty}\left(\mathbb{R}^{n}, A\right)$, com $f(x)=$ $x^{\gamma} e^{i x \xi}, \gamma \in \mathbb{N}^{n}$ dado, e $g \in S^{A}\left(\mathbb{R}^{n}\right)$, então temos que

$$
\int\left[\partial_{x_{j}} f(x)\right] g(x) d x=-\int f(x)\left[\partial_{x_{j}} g(x)\right] d x .
$$

Demonstração. Suponhamos, sem perda da generalidade, que $j=1$. Como $f g \in$ $L^{1}\left(\mathbb{R}^{n}, A\right)$, temos, pelo Teorema de Fubini A.13, que

$$
\begin{gathered}
\int \partial_{x_{1}}[f(x) g(x)] d x=\int_{\mathbb{R}^{n-1}}\left(\int_{\mathbb{R}} \partial_{x_{1}}[f(x) g(x)] d x_{1}\right) d x_{2} \cdots d x_{n}= \\
=\int_{\mathbb{R}^{n-1}}\left(\lim _{R \rightarrow \infty} \int_{[-R, R]} \partial x_{1}[f(x) g(x)] d x_{1}\right) d x_{2} \cdots d x_{n}= \\
=\int_{\mathbb{R}^{n-1}}\left(\lim _{R \rightarrow \infty}\left[f\left(R, x_{2}, \cdots, x_{n}\right) g\left(R, x_{2}, \cdots, x_{n}\right)-f\left(R, x_{2}, \cdots, x_{n}\right) g\left(R, x_{2}, \cdots, x_{n}\right)\right]\right) d x_{2} \cdots d x_{n}=0
\end{gathered}
$$

(ver Observação A.8), pelo corolário A.15. Portanto,

$$
\int\left[\partial_{x_{1}} f(x)\right] g(x) d x=-\int f(x)\left[\partial_{x_{1}} g(x)\right] d x .
$$

Proposição A.17. Seja $A$ uma $C^{*}$-álgebra separável e seja $f \in L^{2}\left(\mathbb{R}^{n}, A\right)$. Então, se $\int f(x)^{*} f(x) d x=0, f(x)=0$, para quase todo $x \in \mathbb{R}^{n}$.

Demonstração. Dado um funcional positivo, $\psi \in A^{*}$, temos que $\psi\left(f(x)^{*} f(x)\right) \geq$ 0. Por outro lado, pela proposição (A.12), $\int \psi\left(f(x)^{*} f(x)\right) d x=0$, o que implica $\psi\left(f(x)^{*} f(x)\right)=0$ q.s.. Como qualquer funcional linear $\varphi \in A^{*}$ é dado por $\varphi=\left(\psi_{1}^{+}-\right.$ $\left.\psi_{1}^{-}\right)+i\left(\psi_{2}^{+}-\psi_{2}^{-}\right)$, onde $\psi_{i}^{+}, \psi_{i}^{-}$são funcionais lineares positivos, $i=1,2([\mathbf{5}$, teorema 3.3.10 e comentários acima]), temos que $\varphi\left(f^{*}(x) f(x)\right)=0$, q.s., $\forall \varphi \in A^{*}$.

Notemos que a bola unitária, $\mathcal{B}$, de $A^{*}$ é compacta na topologia fraca* pelo teorema de Banach-Alaoglu $([6,3.15])$ e portanto, como $A$ é separável, $\mathcal{B}$ é metrizável na topologia fraca* $([\mathbf{6}, 3.16])$. Logo a bola unitária de $A^{*}$ é separável (todo espaço métrico 
compacto é separável). Seja $\mathcal{D} \subseteq \mathcal{B}$ um subconjunto enumerável denso na topologia fraca*.

Vimos que para qualquer $\varphi_{m} \in \mathcal{D}, \varphi_{m}\left(f^{*}(x) f(x)\right)=0$ q.s.. Portanto, o conjunto $E=\left\{x \in \mathbb{R}^{n}: \varphi_{m}\left(f^{*}(x) f(x)\right) \neq 0\right.$, para algum $\left.\varphi_{m} \in \mathcal{D}\right\}$ tem medida nula. Além disso, se $x \notin E$ temos que $\varphi_{m}\left(f^{*}(x) f(x)\right)=0$ para todo $\varphi_{m} \in \mathcal{D}$ e então $\phi\left(f^{*}(x) f(x)\right)=$ 0 para todo $\phi \in \mathcal{B}$. Assim, se $x \notin E, \varphi\left(f^{*}(x) f(x)\right)=0, \forall \varphi \in A^{*}$. Logo, se $x \notin E$, $f(x)=0$; ou seja, $f(x)=0$ para quase todo $x \in \mathbb{R}^{n}$.

Proposição A.18. Mudança de variável. Sejam $T: \mathbb{R}^{n} \rightarrow \mathbb{R}^{n}$ um difeomorfismo $C^{1}$ e $D_{x} T$ a transformação linear dada pela matriz $\left(\frac{\partial T_{i}}{\partial x_{j}}(x)\right)_{i j}$; e seja det $D_{x} T$ o determinante da matriz acima. Se $f \in L^{1}\left(\mathbb{R}^{n}, A\right)$, temos que

$$
\int f(x) d x=\int f \circ T(x)\left|\operatorname{det} D_{x} T\right| d x .
$$

Demonstração. Dado qualquer $\varphi \in A^{*}, \varphi \circ f \in L^{1}\left(\mathbb{R}^{n}\right)$. Logo, por [3, (2.47)], $\int \varphi \circ f(x) d x=\int \varphi \circ f \circ T\left|\operatorname{det} D_{x} T\right| d x$, o que implica que, $\forall \varphi \in A^{*}, \varphi\left(\int f(x) d x\right)=$ $\varphi\left(\int f \circ T\left|\operatorname{det} D_{x} T\right| d x\right)$ e portanto $\int f(x) d x=\int f \circ T\left|\operatorname{det} D_{x} T\right| d x$.

Proposição A.19. Dada uma $C^{*}$-álgebra separável, $A$, temos que $S^{A}\left(\mathbb{R}^{n}\right)$ é denso em $L^{2}\left(\mathbb{R}^{n}, A\right)$.

Demonstração. Vemos em $[\mathbf{3},(8.17)]$ a demonstração de que $S\left(\mathbb{R}^{n}\right)$ é denso em $L^{p}\left(\mathbb{R}^{n}\right)$. A demonstração de que $S^{A}\left(\mathbb{R}^{n}\right)$ é denso em $L^{2}\left(\mathbb{R}^{n}, A\right)$ é feita seguindo os mesmos passos. Primeiro, provamos que as funções contínuas de suporte compacto são densas em $L^{2}\left(\mathbb{R}^{n}, A\right)$. Depois, a demonstração de que as funções $C^{\infty}$ de suporte compacto são densas em $L^{2}\left(\mathbb{R}^{n}, A\right)$ segue por convolução como no caso escalar 


\section{Apêndice B}

Usaremos aqui vários resultados do apêndice $\mathrm{A}$ e, portanto, consideraremos $A$ uma $C^{*}$-álgebra separável.

Definição B.1. Dada $u \in S^{A}\left(\mathbb{R}^{n}\right)$, definimos a Transformada de Fourier, $F$, de $u$, como segue:

$$
F(u)(x)=\int e^{-i x \xi} u(\xi) d \xi, \text { onde } \not \xi=\frac{1}{(2 \pi)^{\frac{n}{2}}} d \xi .
$$

Temos, como no caso escalar ([12, teorema V.1.1]), que $F$ é um operador contínuo de $S^{A}\left(\mathbb{R}^{n}\right)$ em $S^{A}\left(\mathbb{R}^{n}\right)$.

Proposição B.2. F é continuamente inversível de $S^{A}\left(\mathbb{R}^{n}\right)$ em $S^{A}\left(\mathbb{R}^{n}\right)$ e vale que

$$
F^{-1}(u)(x)=\int e^{i x \xi} u(\xi) d \xi, \quad u \in S^{A}\left(\mathbb{R}^{n}\right)
$$

Demonstração. Usaremos a função auxiliar $\phi(x)=e^{-\frac{|x|^{2}}{2}}$. Notemos que $\hat{\phi}(\xi)=\phi(\xi)$ ([12, exemplo V.1.2]), onde $\hat{\phi}$ é a transformada de Fourier (usual) de $\phi$. Tomemos, em $(*), u=F(v)$, para $v \in S^{A}\left(\mathbb{R}^{n}\right)$.

$$
v(x)=\int e^{i x \xi}\left[\int e^{-i y \xi} u(y) d y\right] d \xi
$$

Aqui não podemos trocar a ordem de integração pois $e^{i(x-y) \xi} v(y)$ não é integrável em $\xi$ se $v(y) \neq 0$. Por este motivo, vamos usar a função $\phi$ do seguinte modo:

Seja $\phi_{\varepsilon}(x)=\phi(\varepsilon x)$, para $\varepsilon>0$, dado. É fácil ver que $\hat{\phi}_{\varepsilon}(x)=\varepsilon^{-n} \phi\left(\frac{x}{\varepsilon}\right)$. Assim, aplicando o Teorema de Fubini (A.13),

$$
\int \phi_{\varepsilon}(\xi) e^{i x \xi}\left[\int e^{-i y \xi} v(y) d y\right] d \xi=\int e^{-i y \xi} v(y)\left[\int e^{i x \xi} \phi_{\varepsilon}(\xi) d \xi\right] d y=
$$




$$
\begin{gathered}
=\int v(y)\left[\int e^{-i(y-x) \xi} \phi_{\varepsilon}(\xi) d \xi\right] d y=\int v(y) \hat{\phi}_{\varepsilon}(y-x) d y= \\
\int v(y+x) \hat{\phi}_{\varepsilon}(y) d y=\int v(y+x) \varepsilon^{-n} \phi\left(\frac{y}{\varepsilon}\right) d y=\int v(x+\varepsilon y) \phi(y) d y .
\end{gathered}
$$

E temos $\lim _{\varepsilon \rightarrow 0} \phi_{\varepsilon}(x)=1, \lim _{\varepsilon \rightarrow 0} v(x+\varepsilon y)=v(x)$ e, pelo visto acima:

$$
\int e^{i x \xi} \phi_{\varepsilon}(\xi) F(v)(\xi) d \xi=\int v(x+\varepsilon y) \phi(y) d y .
$$

Por outro lado, $\left\|e^{i x \xi} \phi_{\varepsilon}(\xi) F(v)(\xi)\right\| \leq\|F(v)(\xi)\| \in L^{1}\left(\mathbb{R}^{n}\right)$ e $\|v(x+\varepsilon y) \phi(y)\| \leq$ $\sup \|v(x)\|\|\phi(y)\| \in L^{1}\left(\mathbb{R}^{n}\right)$. $x \in \mathbb{R}^{n}$

Tomando $\varepsilon \rightarrow 0$, pelo Teorema da Convergência Dominada (A.7), temos

$$
\int e^{i x \xi} F(v)(\xi) d \xi=\int v(x) \phi(y) d y .
$$

Como $\int \phi(y) d y=1, v(x)=\int e^{i x \xi} F(v)(\xi) d \xi$, o que prova $(*)$.

A continuidade de $F^{-1}$ se demonstra de modo análogo à continuidade de $F$, ([12, teorema V.1.1]).

Proposição B.3. F é um operador "unitário" em $S^{A}\left(\mathbb{R}^{n}\right)$, com respeito ao produto interno definido no início do capítulo 1.

Demonstração. Dadas $u, v \in S^{A}\left(\mathbb{R}^{n}\right)$, pelo Teorema de Fubini,

$<F(u), v>=\int\left[\int e^{-i x \xi} u(\xi) d \xi\right]^{*} v(x) d x=\int u(\xi)^{*}\left[\int e^{i x \xi} v(x) d x\right] d \xi=<u, F^{-1}(v)>$.

Portanto $F^{*}=F^{-1}$ e $F$ é unitário em $S^{A}\left(\mathbb{R}^{n}\right)$.

Corolário B.4. Se $u \in S^{A}\left(\mathbb{R}^{n}\right)$, então $\|F(u)\|_{2}=\|u\|_{2}$.

Demonstração. $\quad<F(u), F(u)>=<u, F^{-1}(F(u))>=<u, u>$, portanto $\|F(u)\|_{2}=$ $\|u\|_{2}$.

Corolário B.5. Dadas $f, g \in L^{2}\left(\mathbb{R}^{n}, A\right)$, temos que $<F(f), F(g)>=<f, g>$.

Demonstração. Sabemos, pela proposição (3.9) que $L^{2}\left(\mathbb{R}^{n}, A\right) \subseteq \overline{S^{A}\left(\mathbb{R}^{n}\right)}$. Assim, existem seqüências $\left(f_{k}\right)_{k \in \mathbb{N}}$ e $\left(g_{l}\right)_{l \in \mathbb{N}}$ de funções em $S^{A}\left(\mathbb{R}^{n}\right)$ tais que $\lim _{k \rightarrow \infty}\left\|f_{k}-f\right\|_{2}=0$ e $\lim _{l \rightarrow \infty}\left\|g_{l}-g\right\|_{2}=0$. Pelo corolário B.4, $F$ é contínua em $\overline{S^{A}\left(\mathbb{R}^{n}\right)}$ com a norma $\|\cdot\|_{2}$. Assim, pela proposição B.3, $<F(f), F(g)>=\lim _{k, l}<F\left(f_{k}\right), F\left(g_{l}\right)>=\lim _{k, l}<f_{k}, g_{l}>=<$ $f, g>$. 


\section{Referências Bibliográficas}

$[1]$

Marc A. Rieffel, Deformation Quantization for actions of $\mathbb{R}^{d}$, A.M.S. Memoirs, 506, 1993, ix+93pp.

[2] H. O. Cordes, The technique of pseudifferencial operators, London Math. Soc. Lect. N. Series 202, 1995, xii+382pp.

[3] Gerald B. Folland, Real Analysis, J. Wiley \& S. Interscience, 1984, xiv+350pp.

[4] M. Reed \& B. Simon, Methods of Modern Mathematical Physics, I, 1972, xvi+400pp.

[5] Gerard T. Murphy, $C^{*}$-algebras and Operator Theory, Ac. Press, 1990, $\mathrm{x}+286 \mathrm{pp}$.

[6] Walter Rudin, Functional Analysis, Mc-Graw Hill, 1991, xvi+424pp.

[7] K. K. Jensen \& K. Thomsen, Elements of KK-Theory, Birkhäuser, 1991, viii +202 pp.

[8] François Treves, Topological vector spaces, Distributions and Kernels, Ac. Press, 1967, xvi+565pp.

[9] Marc A. Rieffel, Square Integrable Representations of Hilbert Algebras, J. Funct. Analysis 3 (1969) 265-300.

[10] A. P. Calderón \& R. Vaillancourt, On the boundedness of pseudodifferential operators, J. Math. Soc. Japan 23 (1971) 374-378. 
[11] Jörg Seiler, Continuity of Edge and Corner Pseudodifferential Operators, Math. Nach. 205 (1999) 163-182.

[12] Jorge Hounie, Teoria Elementar das Distribuições, $12^{\circ}$ Colóquio Brasileiro de Matemática, IMPA, 1979, vi+164pp.

[13] Donald C. Cohn Measure Theory, Birkhäuser, 1980, x+372pp..

[14] S. T. Melo \& M. I. Merklen, On a conjectured noncommutative BealsCordes-type characterization, A.M.S. Proc. 130 (2002) 1997-2000.

[15] J. J. Kohn \& L. Niremberg, An Algebra of Pseudo-Differential Operators, Comm. Pure Appl. Math. 18 (1965) 269-305.

[16] C. Evans Lawrence, Partial Differential Equations, A. M. S., 1991.

[17] H. O. Cordes, On pseuso-differential operators and smoothness of special Lie-group representations, Manuscripta Math. 28 (1979) 51-69. 